\title{
COUPLED HEAT CONDUCTION AND DEFORMATION IN A VISCOELASTIC COMPOSITE CYLINDER
}

\author{
A Thesis \\ by \\ SNEHA B. SHAH \\ Submitted to the Office of Graduate Studies of \\ Texas A\&M University \\ in partial fulfillment of the requirements for the degree of \\ MASTER OF SCIENCE
}

August 2008

Major Subject: Mechanical Engineering 


\title{
COUPLED HEAT CONDUCTION AND DEFORMATION IN A VISCOELASTIC COMPOSITE CYLINDER
}

\author{
A Thesis \\ by \\ SNEHA B. SHAH \\ Submitted to the Office of Graduate Studies of \\ Texas A\&M University \\ in partial fulfillment of the requirements for the degree of \\ MASTER OF SCIENCE
}

Approved by:

Co-Chairs of Committee, Hanifah Muliana

K. R. Rajagopal

Committee Member, Eyad Masad

Head of Department, Dennis L. O’Neal

August 2008

Major Subject: Mechanical Engineering 


\begin{abstract}
Coupled Heat Conduction and Deformation in a Viscoelastic Composite Cylinder.
\end{abstract}

(August 2008)

Sneha B. Shah, B.E., Gujarat University, India

Co-Chairs of Advisory Committee: Dr. Hanifah Muliana

Dr. Kumbakonam Rajagopal

This study analyzes the thermo-mechanical response of a composite cylinder made up of two layers of linear isotropic viscoelastic materials that belong to the class of non-Thermorheologically Simple Material. The effect of time-varying temperature field due to unsteady heat conduction phenomenon is analyzed on the short term and long term material response in terms of stress, strain and displacement fields. The material properties of the two layers of the composite cylinder at any given location and time are assumed to depend on the temperature at that location at that given instant of time. Sequentially coupled analyses of heat conduction and deformation of viscoelastic composite cylinder is carried out to obtain the overall response. The stress and strain field developed in the composite cylinder is evaluated as the discontinuity in hoop stress and radial strain at the interface of the two layers caused due to mismatch in material properties may lead to delamination if it exceeds critical value. Analytical solution for the stress, strain and displacement fields of the viscoelastic composite cylinder is developed from the corresponding solution of linear elasticity problem by using the Correspondence Principle. The analytical solution for determining the temperature dependent stress, strain and displacement fields is further developed by incorporating the 
temperature dependence on the material properties and modeling the material as nonTSM. To analyze more complex geometry with general loading and boundary conditions, Finite Element(FE) analysis of the composite cylinder is performed and the results of analytical and FE method are found to be in good agreement. Parametric studies are carried out to understand the effect of change in material parameters namely the Prony coefficients in the transient creep compliance, characteristic of creep time in transient creep compliance and the instantaneous elastic compliance, on the overall response of the composite cylinder. The effect of different temperature dependent functions of the material properties, namely linear temperature variation and quadratic polynomial variation on the overall material response is also analyzed. It is observed that the effect of change in elastic properties significantly increases the jump in hoop stress and radial strain. It is also observed that when the materials are highly dependent on temperature the jump in radial strain and hoop stress increases significantly. The radial displacement also increases by a significant amount in both the cases. 


\section{ACKNOWLEDGEMENTS}

I would like to thank my research advisor, Dr. Anastasia Muliana for her patience, guidance and support over the course of this research. She was a constant inspiration, and her assistance and suggestions were invaluable towards the completion of this work. I am really grateful to Dr K R Rajagopal for his very valuable guidance and support all throughtout this work. I am very thankful to him for his helpful insights and invaluable suggestions. I would also like to thank member of my exam committee, Dr. Eyad Masad for his efforts in reviewing and evaluating my research. I would like to acknowledge and thank the Department of Mechanical Engineering at Texas A\&M University for the various opportunities that have helped to make this journey an educational as well as an enjoyable one.

I would like to dedicate this research work to my family. To my parents, who have always encouraged me to pursue my goals, always succeed, and never admit defeat. From them I have learned to be the best that I can be. Thank you for being a part of my life. 


\section{TABLE OF CONTENTS}

ABSTRACT .iii

ACKNOWLEDGMENTS .. $\mathrm{V}$

TABLE OF CONTENTS .................................................................................vi

LIST OF FIGURES................................................................................... viii

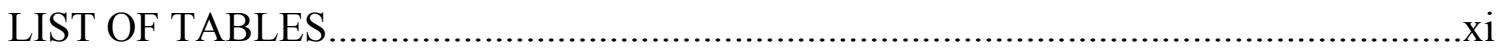

\section{CHAPTER}

I INTRODUCTION

1.1 State of the Art Knowledge on the Thermo-Viscoelastic Behavior of Polymers ............................................................................... 3

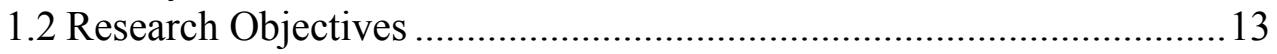

II THERMO-MECHANICAL BEHAVIOR OF A COMPOSITE CYLINDER COMPOSED OF LINEAR ISOTROPIC VISCOELASTIC CONSTITUENTS ..................................................................... 16

2.1 Problem Description...................................................................... 16

2.2 Solution of Viscoelastic Composite Cylinder Using the Correspondence Principle 21

2.3 Temperature Dependent Response of Viscoelastic Composite Cylinder

III ANALYTICAL AND NUMERICAL SOLUTION FOR THERMOMECHANICAL RESPONSE OF THE COMPOSITE CYLINDER

3.1 Analytical Solution of the Field Variables for the Viscoelastic Composite Cylinder

3.2 Finite Element Solution for the Sequentially Coupled Heat Conduction and Deformation in the Viscoelastic Composite Cylinder

3.3 Comparison of Analytical and Finite Element Solution for the Field Variables of the Viscoelastic Composite Cylinder 
IV THE EFFECT OF MATERIAL PARAMETERS IN THE VISCOELASTIC CONSTITUTIVE MODELS ON THE OVERALL RESPONSE OF THE COMPOSITE CYLINDER

4.1 Effect of Varying Prony Coeffiecients in the Creep Compliance on the Overall Response of the Composite Cylinder 56

4.2 Effect of Different Characteristic of Creep Time in the Creep Compliance on the Overall Response 63

4.3 Effect of Different Elastic Constants on the Overall Response of the Composite Cylinder.

4.4 Effect of Temperature Dependent Functions on the Overall Response of the Composite Cylinder.... 75

4.5 Observations .86

V CONCLUSIONS AND FURTHER RESEARCH

5.1 Conclusions .89

5.2 Further Research .91

REFERENCES 92

VITA .96 


\section{LIST OF FIGURES}

FIGURE Page

2.1 Schematic diagram of the composite cylinder of two layers........................... 17

2.2 Composite cylinder made up of two layers .................................................24

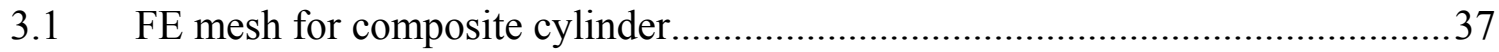

3.2 Effect of initial time increment size on the instantaneous static response of

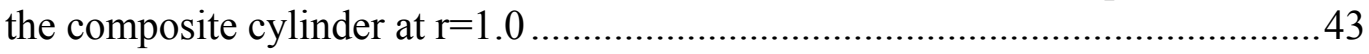

3.3 Finite element mesh convergence study.................................................43

3.4 Effect of time increment on the steady state achieved during transient heat transfer at $\mathrm{r}=1.0$ in the composite cylinder..................................................45

3.5 Evolution of temperature across the body due to heat conduction......................46

3.6 Radial displacement in the composite cylinder as a function of time ................47

3.7 Hoop strain in the composite cylinder as a function of time .............................48

3.8 Hoop stress in the composite cylinder as a function of time............................48

3.9 Radial strain in the composite cylinder as a function of time ..........................49

3.10 Radial stress in the composite cylinder as a function of time ..........................49

3.11 Radial displacement for composite cylinder with temperature dependent properties as a function of time ............................................................5

3.12 Hoop strain for composite cylinder with temperature dependent properties as a function of time

3.13 Hoop stress for the composite cylinder with temperature dependent properties as a function of time

3.14 Radial strain for the composite cylinder with temperature dependent properties as a function of time

3.15 Radial stress for the composite cylinder with temperature dependent properties as a function of time 
FIGURE $\quad$ Page

4.1 Creep compliance of the two layers with time for all three cases .....................58

4.2 Hoop stress for the composite cylinder at different times for different prony

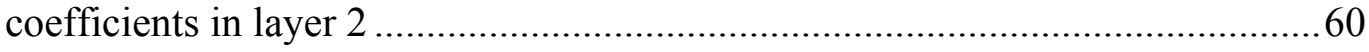

4.3 Radial strain for the composite cylinder at different times for different prony

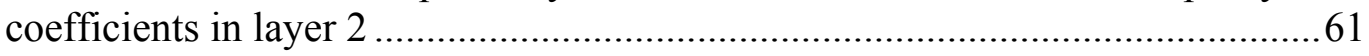

4.4 Radial displacement for the composite cylinder at different times for

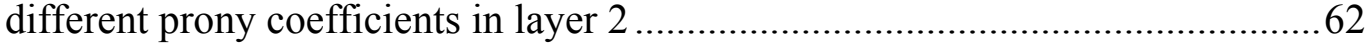

4.5 Creep compliance of both the layers for all three cases .................................65

4.6 Hoop stress for the composite cylinder at different times for different creep

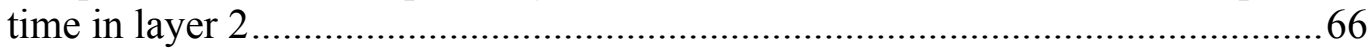

4.7 Radial strain for the composite cylinder at different times for different creep time.

4.8 Radial displacement for the composite cylinder at different times for different creep time

4.9 Creep compliance of layer 1 and layer 2 for all the four cases with time.....

4.10 Hoop stress for the composite cylinder at different times for different elastic part of compliance

4.11 Radial strain for the composite cylinder at different times for different elastic part of compliance 73

4.12 Radial displacement for the composite cylinder at different times for different elastic part of compliance.....

4.13 Hoop stress for the composite cylinder at different times for different temperature dependence of two layers

4.14 Hoop strain for the composite cylinder at different times for different temperature dependence of two layers

4.15 Radial displacement for the composite cylinder at different times for different temperature dependence of two layers ..... 
FIGURE $\quad$ Page

4.16 Hoop stress for the composite cylinder at different times for different temperature dependence (quadratic) of two layers........................................8 82

4.17 Hoop strain for the composite cylinder at different times for different temperature dependence (quadratic) of two layers.......................................... 84

4.18 Radial displacement for the composite cylinder at different times for different temperature dependence (quadratic) of two layers............................. 85 


\section{LIST OF TABLES}

TABLE Page

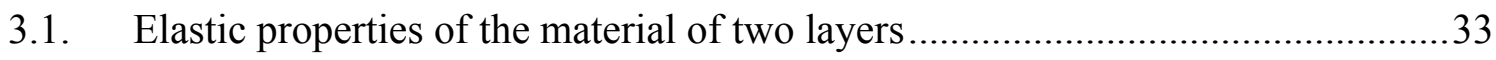

3.2. Elastic compliance and transient properties of the creep compliance for the

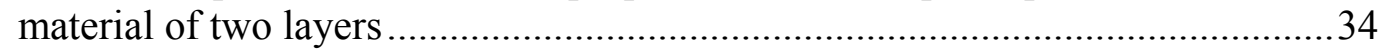

4.1. Material parameters for three different cases by changing the prony

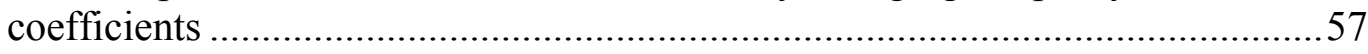

4.2. Elastic and transient material properties of two layers for all the cases .............64

4.3. Transient creep properties for the material of layer 1 and layer 2 in all four

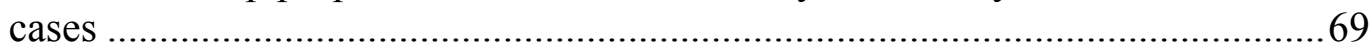

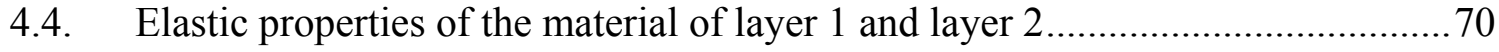

4.5. Elastic properties and transient properties of the creep compliance for the material of layer 1 and layer 2 which are same for case 1 and case 2 


\section{CHAPTER I}

\section{INTRODUCTION}

The use of polymers in engineering applications has been tremendous as the desired properties of polymers can be achieved by altering their macromolecular (chemical) structures. Their low manufacturing cost and light weight also make them very competitive with metals. Polymers are used in the form of layered composites in various applications in aerospace, automotive, offshore and biomedical industries. Understanding the performance of polymers subjected to mechanical loads and exposed to varied environmental effects such as temperature and moisture is essential to increase the reliability of using polymers for structural applications. Polymers exhibit viscoelastic responses. The viscoelastic behavior is often intensified at higher stress levels and extreme environmental conditions.

Many studies have been done to investigate the effect of temperature changes on the material properties like creep and relaxation characteristics of viscoelastic materials. In some studies, a series of isothermal creep or relaxation tests are carried out. A shift factor, which is a temperature dependent material property, denotes the amount of time shifting required to shift relaxation/creep moduli at certain temperature to the ones at the reference temperature. The shift factors can be used to predict the material responses at other non-reference temperatures. This type of materials is categorized as Thermorheologically Simple Material (TSM). A more general class of materials that

This thesis follows the style of Composite Science and Technology. 
does not belong to TSM constitutes a class known as non-thermorheologically simple materials.

The present study analyses the effect of temperature changes on mechanical response of a composite cylinder comprised of two layers of isotropic viscoelastic materials. The composite cylinder is subjected to the internal pressure and external pressure on its innermost and outermost boundaries respectively and also a temperature field on its outermost boundary. The studied viscoelastic materials belong to the class of non-thermorheologically simple material. It is assumed that the material properties at any particular location in the viscoelastic body depend on the temperature at that location. Since in this study, the materials comprising the inner and the outer layer are viscoelastic, their response to the thermo-mechanical loading is history dependent. Therefore, a sequentially coupled analysis of heat conduction and deformation is performed to evaluate the overall response of the viscoelastic composite cylinder. The mechanical response in terms of stress, strain and displacement field is evaluated as higher amount of discontinuity in stress and strain field at the interface of two layers that arises due to mismatch in material properties may lead to delamination if exceeded beyond a certain value. In problems of complex geometry and complex boundary conditions, it is not always possible to obtain an exact closed form analytical solution. For that purpose, a numerical analysis based on finite element (FE) approach is carried out to study the response of the composite structures. A time-integration algorithm is formulated to link the constitutive model to the general FE framework. 
This chapter presents literature review of analytical, experimental and numerical works done on thermo-viscoelastic behavior of polymeric materials, followed by research objectives.

\subsection{STATE OF THE ART KNOWLEDGE ON THE THERMO-VISCOELASTIC BEHAVIOR OF POLYMERS}

\subsubsection{ANALYTICAL AND EXPERIMENTAL STUDIES ON THERMO- VISCOELASTIC BEHAVIOR OF POLYMERS}

The use of polymers in many industrial applications has led to the need of understanding the polymer behaviors under general loading conditions and extreme environmental conditions. The short term and long term behavior of polymers are significantly altered when subjected to temperature or moisture fluctuations. Hence, many analytical as well as experimental studies have been carried out to gain detailed understanding of these effects on the overall performance of polymers.

For a large class of problems in linear viscoelasticity, a Correspondence Principle has been widely used, which enables the solution to a problem in linear viscoelasticity to be constructed from the solution to a corresponding problem in linear elasticity. Alfrey [1] discussed two types of boundary value problems in linear viscoelasticity, the first one in which the tractions are given as the function of space and time variables for all the times and the second type in which the displacement at the boundary is given as the function of space and time variables for all the times considered. He showed that for both types of boundary value problems the solution for the stress and displacement fields developed in the body is reduced to the solution of equivalent boundary value problems 
of elasticity. Lee et al. [2] discussed the method of stress analysis in bodies comprising of linear viscoelastic materials based on the Laplace Transform method. They discussed that when the body under consideration has boundaries that remain invariant with time and also the prescribed traction and displacement conditions on the boundary do not vary with time, and undergoes infinitesimal displacement, then by applying the Laplace Transform, problems can be reduced to the corresponding problem in linear elasticity and the stress field can be obtained by subsequent Laplace inversion of the elastic problem. They discussed the example of determination of stress field in a thick viscoelastic cylinder with an elastic shell, the outer boundary being subjected to internal pressure, using this Laplace Transform method. Gurtin and Stenberg [3] discussed the constitutive relations for the linear isotropic viscoelastic materials using the convolution relation. They discussed the general integral representation for the stress and strain constitutive law expressed in the relaxation form or creep integral form including the jump discontinuities. They discussed the mixed boundary value problem in the quasistatic linear theory of viscoelasticity and showed that the initial response of a viscoelastic solid is elastic. They also discussed the solution of boundary value problem using the Laplace Transform method. Lee and Rogers [4] discussed the solution of stress analysis for more general problems in the linear viscoelastic materials by discussing the constitutive relation given via an integral operator. The time dependent parameters can be characterized using relaxation modulus or creep compliance directly measured experimentally for a finite time range. The proposed integral operator form did not pose any limitation of fixed boundary or types of boundary conditions as posed 
by the Laplace Transform method. More general problems could be studied by this method which are not amenable to the Laplace Transform method. Rajagopal and Wineman [5] discussed the generalization of the Correspondence Principle between linear elasticity and linear viscoelasticity established for classical Linear Viscoelastic (LV) solid model to Quasi-Linear Viscoelastic (QLV) model, which they refer to as Quasi-Correspondence Principle. They discussed the cases of QLV model undergoing homogeneous uniaxial elongation and torsion and showed that the correspondence between LV and QLV models is applicable in these cases. They also discussed case when this correspondence between LV and QLV models breaks down by showing an example of bending. Though the Correspondence Principle has been gainfully used to solve many problems in viscoelasticity, there are certain situations when it breaks down. Rajagopal and Wineman [6] discussed in detail the conditions under which the Correspondence Principle breaks down. They discussed that all the material points $\mathbf{x}$ must be a part of the body for all times $\mathrm{t} \geq 0$ for applicability of the Correspondence Principle. If any of the boundary is ablating or burning away like in a solid fuel propellant then, displacement is not defined for all times $t \geq 0$ as the particle $\mathbf{x}$ of the body has already burned away and the Correspondence Principle is no more applicable. Another condition when it breaks down is, when at a fixed point on the boundary, boundary condition changes from specified displacement to specified surface traction during the deformation or vice versa. i.e. the type of boundary condition specified changes with time. Rajagopal and Srinivasa [7] showed that the Correspondence Principle proposed by Schapery [8] for problems of non-linear viscoelastic materials 
undergoing large deformations does not satisfy the balance of angular momentum. They discussed an example of Hadamard compressible material undergoing simple shear deformation to show the inapplicability of the Correspondence Principle to nonlinear viscoelastic materials undergoing large deformations.

While viscoelastic materials exhibit stress relaxation phenomenon, inherent viscoelasticity is not the only reason for it. Rajagopal and Wineman [9] discussed that the materials can exhibit stress relaxation behavior due to variety of reasons like ageing or inherent viscoelastic nature and both stress relaxation phenomena are distinctly different. They showed with an example of torsion of viscoelastic body capable of ageing that the stress relaxation due to classical viscoelastic nature and due to ageing can be delineated. The material that they considered exhibited characteristic of faster ageing when exposed to higher strains and also faster ageing when subjected to same strains for longer time. Thus they showed that classical viscoelasticity and ageing are distinct phenomena.

Rajagopal et al. [10] developed a thermodynamic framework to describe a transition of polymer melt to an elastic solid that happens over a narrow range of temperatures. They studied the problem of fiber spinning in polymers like Polyethylene Terephthalate that are amorphous below the glass transition temperature and showed that the theoretical results predicted by their theory were in good agreement with the experimental results. Rajagopal and Kannan [11] developed a thermo mechanical framework to describe the transition from a viscoelastic fluid to a viscoelastic solid 
which was a generalization of [10]. They discussed the kinetics associated with the transition and the change of the response characteristics during the transition.

The viscoelastic behavior of the polymers strongly depends on the environmental conditions, i.e. temperature and moisture to which it is subjected. Several studies have been carried out to establish stress-strain-temperature-time relations. For a class of linear viscoelastic materials time-temperature superposition principle (TTSP), as discussed by Leaderman [12], is used to describe the effect of temperatures on the responses of viscoelastic materials. This principle is approximately valid for amorphous polymers. The main implication of the TTSP is that material property (stress relaxation or creep compliance functions) at any temperature can be expressed in terms of the material property at reference temperature and another material property known as timeshift function. The method involves creating a master curve by shifting the relaxation moduli or creep compliance data obtained from short-term test duration at several elevated temperatures to the one at the reference temperature by horizontal time shifting along a logarithmic time scale. Schwarzl and Staverman [13] discussed the applicability of time-temperature correspondence principle to various classes of linear viscoelastic materials. They proposed that if the change in the viscoelastic material behavior due to temperature is completely equivalent to a corresponding shift of the logarithmic time scale then it is categorized as Thermorheologically Simple Material (TSM). The viscoelastic materials which do not fall in this category form another class known as Thermorheologically Complex Materials (TCM). In TSM, the temperature dependent viscoelastic response can be characterized by one function of temperature, which is time 
shift factor. It was discussed that in TSM the same molecular events took place but with different speeds at different temperatures while in TCM sequence of molecular events vary at different temperatures. Lee and Morland [14] studied the stress-analysis in the linear viscoelastic materials subjected to varying temperature fields. They showed that material characteristic functions like stress relaxation or creep compliance at any arbitrary uniform temperature can be expressed in terms of creep/relaxation behavior at the reference temperature by shifting the responses to a new time scale, known as reduced time. They analyzed the effect of temperature changes on the overall stress fields of TSM assuming the material incompressible and thus not considering any thermal stresses that would arise during the transient temperature stage. In particular they analyzed the plain strain problem of a hollow cylinder subjected to internal pressure and constant temperature difference across the cylinder. Christensen and Naghdi [15] derived constitutive laws, dissipation function and the related thermodynamic restrictions for small non-isothermal deformation of linear viscoelastic materials. They used a representation of Helmholtz free energy and derived the constitutive laws for stress, entropy, and heat flux for continuous histories and also extended the same to non continuous kinematic and thermal histories.

Many experiments were also carried out to study the time-dependent behavior of viscoelastic materials. Creep tests and relaxation tests at various temperatures were conducted to study the thermo-mechanical and long term responses of the viscoelastic materials. Tobolsky and Catsiff [16] presented a summary of a series of stress relaxation experiments carried on polyisobutylene specimens. It was shown that the data of stress 
relaxation experiments carried out at various higher temperatures on the polyisobutylene does satisfy the time-temperature superposition principle when temperatures are close to the glass transition temperature. Lai and Findley [17] conducted uniaxial tension creep tests on polyurethane specimens in the non linear range at varying temperatures. They proposed two different methods based on a modified superposition principle to account for varying temperature. One method consisted of a shift factor which is temperature and stress dependent. The other involved stress and temperature dependent function of strain. Ward and Onat [18] carried out tests to study isothermal extensional deformations of oriented polypropylene monofilament subjected to time-dependent loadings. They further discussed that the elongation at a given time is a non-linear functional of the stress-rate history to which the filament is subjected prior this instance. They also showed that the experimental results could be represented within reasonable accuracy by this functional of stress-rate history.

Tschoegl and Fesko [19] studied the nature of the time-temperature superposition that can be applied to the Thermorheologically Complex Material and proposed a simple model applicable to two-phase materials. They proposed that the shift factor should not only be a function of temperature but also of time or frequency so as to characterize TCM. They used an additive compliance model which assumes that the compliance of the two phase materials have parallel coupling and thus is additive, to obtain the required time-temperature relations for superposition. Caruthers and Cohen [20] carried out computer simulations on TCM at different temperatures instead of doing laboratory testing so as to bring out the complete nature of thermorheological complexities. They 
used an additive compliance model similar to that of Fesko and Tschoegl [15] and generated creep compliance isotherms. Their model also predicted time and temperature dependent shift factor applicable to the entire domain. Weitsman [21] developed an experimental scheme for characterizing thermo-viscoelastic properties of adhesives and composites within linear range of stress-strain response. He demonstrated that the isothermal creep data may not be adequate to characterize the thermoviscoelastic response of TCM. He also emphasized on the importance of the complete thermoviscoelastic characterization by using the experimental creep and recovery data of FM73 adhesive and showed that in-complete characterization can give rise to discrepancies of up to 30\%. Harper and Weitsman [22] proposed a characterization method for a class of TCM and demonstrated it by conducting experiments on Hercules 3502 epoxy resin. Their technique included temperature effects by incorporating a vertical and horizontal shift factors which were temperature dependent. They demonstrated that the characterization method that used only the data of isothermal creep tests showed large discrepancies of about $17 \%$ where as the method that was based on the results of the transient temperature and isothermal creep tests together showed good agreement with experimental data. Knauss and Zhu [23] studied the non-linear thermo-mechanical creep behaviors of polycarbonate at temperatures between 0 to $140^{\circ} \mathrm{C}$ under pure shear loading conditions. The shear creep in the linear viscoelastic range was measured for reference. The shear creep tests were conducted in the non linear viscoelastic range on the Arcan specimens at different temperatures and different stress levels. It was shown 
that the nonlinearity started at around $1 \%$ of strains at all temperatures. It was shown that time-temperature superposition was not applicable in the non-linear range.

\subsubsection{NUMERICAL APPROACH WITHIN THE FE FRAMEWORK FOR THERMO-VISCOELASTIC BEHAVIOR OF POLYMERIC MATERIALS}

The approach involving numerical techniques compatible with Finite Element Analyses has evolved in last few decades for analyzing viscoelastic response of polymeric materials. Zienkiewicz et al. [24] developed an incremental algorithm for simulating creep behavior of quasi-static problems. They used a constitutive model consisting of series of Kelvin elements coupled with an elastic spring. The strain histories were stored only for the current time and strain rate was modeled as function of current stress and accumulated creep strains. Taylor et al. [25] proposed a recurrence numerical algorithm for solution of uncoupled, quasi-static boundary value problem for a linear viscoelastic solid undergoing thermal and mechanical deformation. The recursive algorithm formulated the current stress tensor as a function of the history variables stored at the previous time step, current time increment and the current strain increments. Feng [26] considered viscoelastic constitutive equations in integral form and developed a recursive formula to evaluate the value of convolution integral at the current time using the values from the previous time step. His recursive formulation was similar to that of Taylor [25]. He applied this recursive formula to the constitutive equation of linear viscoelastic material described by Boltzman's superposition integral equation as a special case. Henriksen [27] developed a recursive numerical algorithm for analyzing the non-linear viscoelastic materials. The non-linear viscoelastic behavior 
was mainly due to high stress magnitudes. He used single integral constitutive law that was proposed by Schapery to describe the material. In his approach, strain was defined by a stress operator that included instantaneous compliance and hereditary strain which was updated by recursive computation. Lai and Bakker [28] developed a 3-D constitutive model based on 1-D representation given by Schapery for non-linear viscoelastic materials that incorporated temperature and physical ageing effects through reduced time. Their model assumed deviatoric and hydrostatic responses to be uncoupled. They expressed the constitutive equation in incremental form and employed recursive computation to update the values of hereditary integrals at end of each time step. They integrated this model to Finite Element framework and showed that numerical predictions were within good agreement with the experimental data of glassy amorphous polymer PMMA. Haj-Ali and Muliana [29] formulated a recursive-iterative algorithm for non-linear isotropic viscoelastic materials represented by Schapery's model with stress-based state variables. The constitutive equations were expressed in incremental form for each time step and constant incremental strain rate was assumed that was compatible with general displacement based FE network. The numerical formulation derived by Taylor et al. [25], Henriksen [27], and Lai and Bakker [28] was used to update the linearized stress part. Muliana and Khan [30] developed a combined recursive-iterative method for nonlinear thermo-viscoelastic analyses of isotropic polymers belonging to the class of TCM. The time-integration algorithm developed could incorporate the stress and temperature dependence on the initial, long term and transient material properties. Two types of iterative procedures based on Fixed point 
method and Newton-Raphson method were examined within the recursive algorithm. A consistent tangent stiffness matrix was formulated to accelerate the convergence at the material level. The proposed numerical algorithm was verified using the thermoviscoelastic experimental data of Harper and Weitsman [22].

\subsection{RESEARCH OBJECTIVES}

This study analyzes the effect of temperature during transient heat transfer process on the deformations of the composite cylinder made up of two linear isotropic viscoelastic materials that belong to the class of non-thermorheologically simple material. Time and temperature dependent constitutive model is used to characterize two materials. It is assumed that the material properties at any particular location at any given time in the viscoelastic body depends on the temperature at that location at that given instant of time. The elastic part, transient part and the characteristics of creep time in the time dependent compliances of the two materials are temperature dependent. Sequentially coupled analyses of heat conduction and deformation are carried out to predict the overall response of the viscoelastic composite cylinder. For problems with complex geometry, loading histories, and boundary conditions, it is not always possible to obtain an exact closed form analytical solution. It is due to this reason that a numerical analysis based on FE approach is carried out to study the response of the composite cylinder. A time-integration algorithm is then formulated to implement the constitutive viscoelastic model in a general FE framework. A parametric study is carried out to study the effect of the different parameters such as temperature dependent elastic part, transient part, and the characteristics of creep time of the creep compliance on the 
displacement, stress and strain fields developed in the body. The outline of this thesis is described as follows.

Chapter II presents a sequentially coupled analysis of heat conduction and deformation of a composite cylinder consisting of two layers of linear isotropic viscoelastic materials. The constitutive relations are introduced and the appropriate governing equations along with the boundary and initial conditions for the deformation and conduction of heat are discussed. The analytical solution for the stress, strain and displacement field is obtained from the corresponding problem in linear elasticity by using the Correspondence Principle. The effect of temperature is incorporated by considering material properties at any location to depend on the temperature at that location. The solution for determining the temperature dependent stress, strain and displacement fields is developed by incorporating the temperature dependence on the material parameters and modeling the material as Thermorheologically Simple Material (TSM) and then as non TSM.

Chapter III describes the geometry, material parameters and loading conditions to which the composite cylinder is subjected. The finite element (FE) method is employed to analyze the overall response of the composite cylinder. FE software ABAQUS is used to obtain the solution for the heat conduction and the deformation of the composite cylinder. The convergence study is also carried out to ensure that the time increment and the finite element mesh gives numerical results within adequate accuracy. The analytical and the FE solutions for the field variables of stress, strain and displacement are compared. 
Chapter IV presents the parametric study carried out to understand the effect of different parameters on the overall response of the viscoelastic composite cylinder. The effect of each individual material parameter namely instantaneous elastic part, Prony coefficients of the transient part of creep compliance and characteristic of creep time, on the stress, strain and displacement fields is studied. In addition, the effect of temperature dependent functions i.e. linear and quadratic polynomial forms of temperature dependence of the material properties, on the overall response of the composite cylinder is investigated.

Chapter V describes the conclusions and further research. 


\section{CHAPTER II}

\section{THERMO-MECHANICAL BEHAVIOR OF A COMPOSITE CYLINDER COMPOSED OF LINEAR ISOTROPIC VISCOELASTIC CONSTITUENTS}

This chapter presents a sequentially coupled analysis of heat conduction and deformation of a composite cylinder consisting of two layers of linear isotropic viscoelastic materials. The constitutive relations are introduced and the appropriate governing equations along with the boundary and initial conditions for the viscoelastic deformation and conduction of heat are discussed. The method involving Correspondence Principle is described to obtain the solution of viscoelastic problem from the corresponding problem in linear elasticity. The effect of temperature is incorporated by considering material properties at any location to depend on the temperature at that location. The solution for determining the temperature dependent stress, strain, and displacement fields is developed by incorporating the temperature dependence on the time-dependent material constants and modeling the material as Thermorheologically Simple Material (TSM) and then as non-TSM.

\subsection{PROBLEM DESCRIPTION}

In this section the geometry of the composite cylinder along with the prescribed boundary and initial conditions is described. A layered hollow composite cylinder consisting of two linear isotropic homogeneous non ageing viscoelastic materials subjected to a temperature field on its outer boundary is considered. The effect of temperature dependence on the response of composite cylinder is studied by considering 
the material properties at a particular location to be dependent on the temperature at that location. The coupled problem of conduction of heat and deformation is solved simultaneously.

The composite cylinder is subjected to pressure $p_{o}$ on its outer boundary and $p_{i}$ on its inner boundary as shown in Fig. 2.1. The pressure $p_{o}$ is arbitrarily chosen to be higher than the pressure $\mathrm{p}_{\mathrm{i}}$. However any value of the pressure could have been chosen. It is assumed that the heat conduction takes place across the body in the radial direction from the outer boundary towards inner boundary due to temperature condition imposed on the outermost boundary. Once the entire body reaches the steady state of temperature, it is assumed to remain in that state thereafter. The bonding at the interface between the two layers is assumed to be sufficiently strong and to have a traction continuity and displacement continuity at all the times.

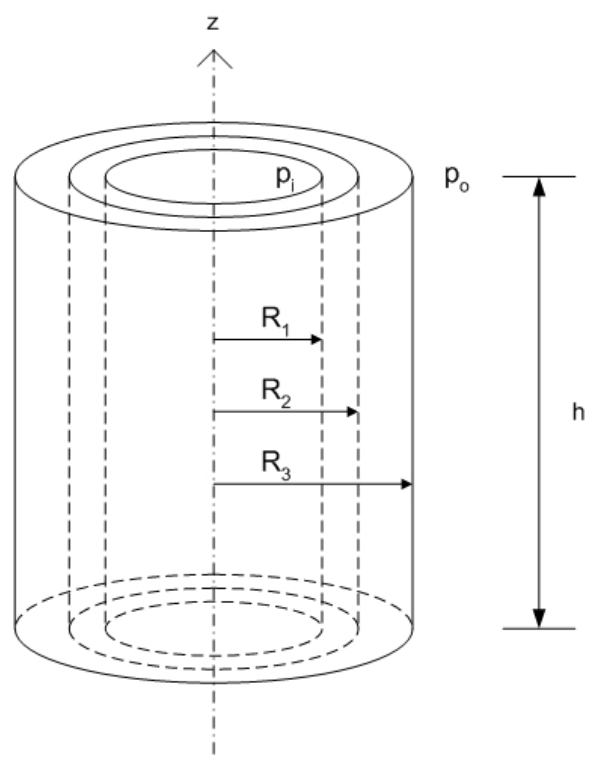

Fig. 2.1. Schematic diagram of the composite cylinder of two layers 
The problem of radial heat transfer across the composite body is studied under the assumption that all the heat transfer occurs due to the conduction of heat across the solid body and convection and radiation effects are not considered. In order to understand the effect of temperature dependent material properties on the overall response of the composite cylinder, the problem of radial heat conduction, balance of linear and angular momentum and the constitutive equations are solved simultaneously. The phenomenon of heat conduction is assumed to be governed by the Fourier's heat conduction law in the radial direction. All the time dependent terms in the heat conduction equation are retained so as to study the manner in which the heat is conducted along the body resulting in the temperature change in the composite body, but the inertial effects in the motion of the composite with regard to the balance of linear momentum are neglected and thereby only the equilibrium equations of the motion are considered. The equations for the conduction of heat and the motion of the composite are coupled as the material properties depend on the temperature at that material point.

The composite cylinder is made up of two viscoelastic solid materials and hence it deforms continuously in time due to existence of constant stresses. The attention is restricted to small deformation gradient problems. Let $x_{i}, i=1,2,3$ be the coordinates of a material particle in the undeformed state. $u_{i}(x, t), i=1,2,3$ denote the components of displacement of the material particle at $\mathbf{x}$ at time t. $\varepsilon_{\mathrm{ij}}(\mathbf{x}, \mathrm{t})$ denote the strain history and $\sigma_{\mathrm{ij}}(\mathbf{x}, \mathrm{t})$ denote the Cauchy stress history for the particle at $\mathbf{x}$ at time $\mathrm{t}$. It is assumed that the composite cylinder is initially undisturbed,

$u_{i}(x, t)=\varepsilon_{i j}(x, t)=\sigma_{i j}(x, t)=0, \forall t<0$ 
$\mathrm{T}(\mathbf{x}, \mathrm{t})$ denotes the temperature field at the material point $\mathbf{x}$ at time $\mathrm{t}$. All the quantities are expressed in the cylindrical polar co-ordinate system $(\mathrm{r}, \theta, \mathrm{z})$. The equation governing the phenomenon of conduction of heat has the following form:

$\frac{\partial T^{(j)}}{\partial t}=K^{(j)} \Delta T^{(j)}$

$\mathrm{j}=1,2$ corresponds to the two layers of the composite

where $K$ is the thermal conductivity of the material of the $\mathrm{j}^{\text {th }}$ layer which is assumed to be a constant scalar. In a more general case thermal conductivity $\mathrm{K}$ can depend on the position and temperature but in this study it is assumed to be a constant. Layer 1 is the inner layer, while layer 2 is the outer layer. $\Delta$ denotes the Laplacian operator. In the cylindrical coordinates this equation becomes:

$\frac{\partial T^{(j)}}{\partial t}=K^{(j)}\left[\frac{1}{r} \frac{\partial}{\partial r}\left(r \frac{\partial T^{(j)}}{\partial r}\right)+\frac{1}{r^{2}} \frac{\partial^{2} T^{(j)}}{\partial \theta^{2}}+\frac{\partial^{2} T^{(j)}}{\partial z^{2}}\right]$

The displacement field at any material point is assumed to be of the following form:

$\mathbf{u}^{(j)}=\mathbf{u}^{(j)}(r, \theta, z, t)$

As mentioned earlier, while considering the equation of motion, the inertial effects involved are neglected and the equilibrium equations in absence of body forces are considered as:

$\operatorname{div} \boldsymbol{\sigma}^{(j)}=\mathbf{0}$

where div denotes the divergence operator. The linear strain and displacement relations are considered to be of the form:

$\boldsymbol{\varepsilon}^{(j)}=\frac{1}{2}\left(\operatorname{grad} \mathbf{u}^{(j)}+\left[\operatorname{grad} \mathbf{u}^{(j)}\right]^{\mathrm{T}}\right)$ 
where grad denotes the gradient operator.

The initial and boundary conditions used to solve the heat conduction equation and the displacement fields are:

$$
\begin{aligned}
& T^{(2)}\left(R_{3}, \theta, z, t\right)=T_{1}(t), \forall t \geq 0 \\
& T^{(1)}(r, \theta, z, 0)=0, \forall R_{1} \leq r \leq R_{2} \\
& T^{(2)}(r, \theta, z, 0)=0, \forall R_{2} \leq r<R_{3} \\
& \frac{\partial T^{(1)}}{\partial r}\left(R_{1}, \theta, z, t\right)=0 \\
& \sigma_{r r}^{(1)}\left(R_{1}, \theta, z, t\right)=-p_{i} \\
& \sigma_{r r}^{(2)}\left(R_{3}, \theta, z, t\right)=-p_{o}
\end{aligned}
$$

The bonding between the two layers is assumed to be sufficiently strong and the following conditions of continuity are imposed on the interface. Firstly it is assumed that the displacement field at each point at the interface is continuous resulting in:

$$
\mathbf{u}^{(1)}\left(R_{2}, \theta, z, t\right)=\mathbf{u}^{(2)}\left(R_{2}, \theta, z, t\right)
$$

Second, the continuity of temperature and traction at the interface between the two layers is enforced:

$$
\begin{aligned}
& T^{(1)}\left(R_{2}, \theta, z, t\right)=T^{(2)}\left(R_{2}, \theta, z, t\right) \\
& \sigma_{r r}^{(1)}\left(R_{2}, \theta, z, t\right)=\sigma_{r r}^{(2)}\left(R_{2}, \theta, z, t\right)
\end{aligned}
$$

In the case of the composite cylinder of finite length, the condition of traction continuity at the interface also implies $\sigma_{r z}^{(1)}\left(R_{2}, \theta, z, t\right)=\sigma_{r z}^{(2)}\left(R_{2}, \theta, z, t\right)$. Using the condition of the continuity of the heat flux at the interface we also get: 


$$
K^{(1)} \frac{\partial T^{(1)}}{\partial r}\left(R_{2}, \theta, z, t\right)=K^{(2)} \frac{\partial T^{(2)}}{\partial r}\left(R_{2}, \theta, z, t\right)
$$

The temperature condition imposed at the outer most boundary of $r=R_{3}$ is

$$
T_{1}(t)= \begin{cases}0 & \text { if } t<0 \\ 1 & \text { if } t \geq 0\end{cases}
$$

\subsection{SOLUTION OF VISCOELASTIC COMPOSITE CYLINDER USING THE CORRESPONDENCE PRINCIPLE}

For a class of problems in linear viscoelasticity, a general method has been developed which enables to solve problems in linear viscoelasticity from the solution of the corresponding problem in linear elasticity known as Correspondence Principle as discussed by Christensen [31] and Rajagopal and Wineman [6]. Consider a body that is initially undisturbed, in that the displacement, strain and stress fields are:

$u_{i}(x, t)=\varepsilon_{i j}(x, t)=\sigma_{i j}(x, t)=0, \forall t<0$

For all the times $t \geq 0$, the linearized strain-displacement relation, the balance of angular momentum which implies that stress tensor is symmetric at each instant of time and the balance of linear momentum in absence of inertial effects and body force, are given as:

$$
\begin{aligned}
& \varepsilon_{i j}=\frac{1}{2}\left(\frac{\partial u_{i}}{\partial x_{j}}+\frac{\partial u_{j}}{\partial x_{i}}\right) \mathrm{i}, \mathrm{j}=1,2,3 \\
& \sigma_{i j}=\sigma_{j i} \\
& \frac{\partial \sigma_{i j}}{\partial x_{j}}=0
\end{aligned}
$$

At each point of the surface either displacement or traction boundary conditions are specified for all times $t \geq 0$. It is assumed that the boundary condition at a point, 
whether it is prescribed traction or displacement, does not change with time. That is, at a point on the boundary, displacement should not be specified for some part of time with traction condition specified for the rest of the time.

For the problems in linear viscoelasticity that satisfy the above conditions, a Laplace Transform of the governing field equations and the boundary conditions will result in the similar set of equations as that of the corresponding boundary value problem for the equilibrium state of a linear elastic material. This means that the Laplace transformed viscoelastic solution can be directly obtained from the solution of the corresponding elastic problem by multiplying the Laplace transform parameter to the Laplace Transform of the material properties and interpreting the elastic field variables as the transformed field variables. The viscoelastic solution is then obtained by inverting the transformed solution back to the real time domain. This generalized method that involves the replacement of elastic material properties by appropriate forms of transformed viscoelastic properties and elastic field variables as transformed viscoelastic field variables and then followed by the inversion of the transformed equations to get the final solution of boundary value problem in viscoelasticity is known as the Correspondence Principle.

However, there are certain cases when the Correspondence Principle breaks down. As discussed in chapter I, Wineman and Rajagopal [6] have discussed in detail the cases when the Correspondence Principle is no more applicable. The studied composite cylinder does satisfy all the conditions necessary for the applicability of the Correspondence Principle, which is used to construct the solution of viscoelastic stress 
and deformation fields developed on application of pressure on the boundaries on the cylinder. The solution of the problem was constructed for the linear isotropic elastic composite cylinder subjected to same boundary conditions. The solution for the deformation in viscoelastic cylinder was obtained using the Correspondence Principle.

For simplicity, the problem of plane strain conditions is considered by assuming the annular cylinder to be infinitely long. Since the cylinder is assumed to be infinitely long the displacement and the temperature fields are considered to be independent of the $\mathrm{z}$ coordinate. Also the displacement and temperature fields are taken to be symmetric with respect to the axis of the cylinder and so the displacement and temperature can be assumed of the form:

$$
\begin{aligned}
& \mathbf{u}^{(j)}=u_{r}^{(j)}(r, t) \mathbf{e}_{r} \\
& T^{(j)}=T^{(j)}(r, t)
\end{aligned}
$$

Thus the equation governing the conduction of heat through the composite cylinder now takes the form:

$$
\frac{\partial T^{(j)}}{\partial t}=K^{(j)}\left[\frac{1}{r} \frac{\partial}{\partial r}\left(r \frac{\partial T^{(j)}}{\partial r}\right)\right]
$$

The initial, boundary and interface conditions used are:

$$
\begin{aligned}
& T^{(2)}\left(R_{3}, t\right)=T_{1}(t), \forall t \geq 0 ; \quad T^{(1)}(r, 0)=0, R_{1} \leq r \leq R_{2} ; \quad T^{(2)}(r, 0)=0, R_{2} \leq r<R_{3} \\
& K^{(1)} \frac{\partial T^{(1)}}{\partial r}\left(R_{2}, t\right)=K^{(2)} \frac{\partial T^{(2)}}{\partial r}\left(R_{2}, t\right) ; \quad \frac{\partial T^{(1)}}{\partial r}\left(R_{1}, t\right)=0 ; \quad \sigma_{r r}^{1}\left(R_{2}, t\right)=\sigma_{r r}^{2}\left(R_{2}, t\right) \\
& u_{r r}^{1}\left(R_{2}, t\right)=u_{r r}^{2}\left(R_{2}, t\right) ; \quad \sigma_{r r}^{2}\left(R_{3}, t\right)=-p_{o}, \sigma_{r r}^{1}\left(R_{1}, t\right)=-p_{i}
\end{aligned}
$$


The solution of composite elastic cylinder is developed using the method employing the Airy Stress Function technique as discussed by Sadd [32]. As shown in the Fig. 2.2 below, the composite cylinder is thought of as being made up of two separate elastic cylinders having same pressure $p_{c}$ (which is to be determined) at the interface i.e at $\mathrm{R}=\mathrm{R}_{2}, \sigma_{r r}^{1}\left(R_{2}\right)=\sigma_{r r}^{2}\left(R_{2}\right)=-p_{c}$
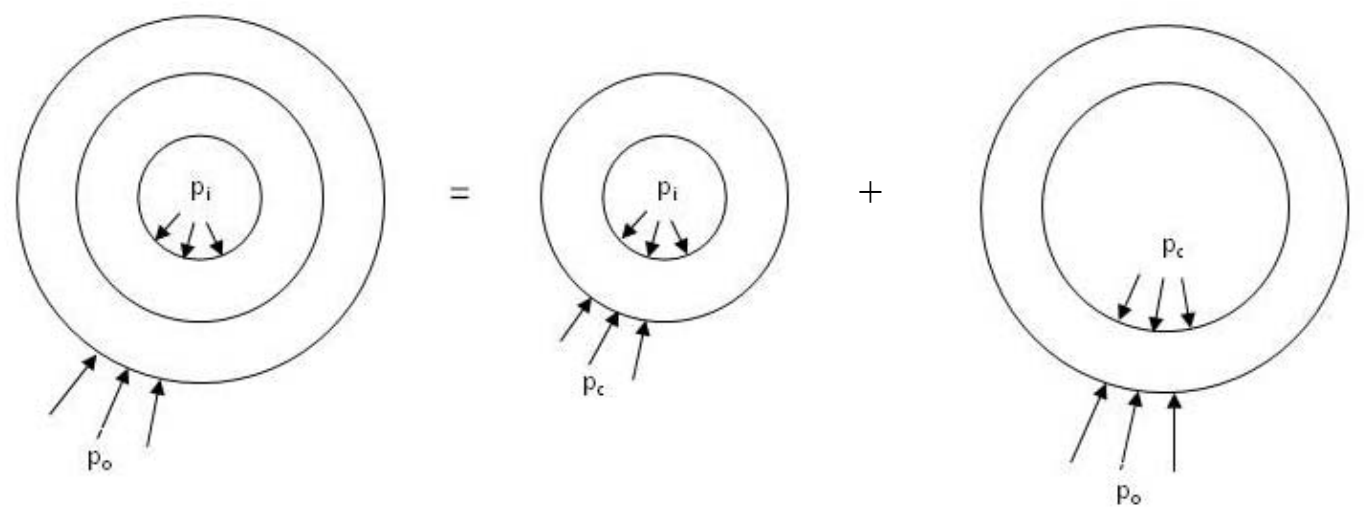

Fig. 2.2. Composite cylinder made up of two layers

The non zero stresses and the radial displacements in the two cylinders are taken of the following form: ( Sadd [32] )

$$
\begin{gathered}
\sigma_{r r}^{1}(R)=\frac{A}{R^{2}}+B, \quad R 1 \leq R \leq R 2 \\
\sigma_{r r}^{2}(R)=\frac{C}{R^{2}}+D, \quad R 2 \leq R \leq R 3
\end{gathered}
$$




$$
\begin{aligned}
& u_{r r}^{1}(R)=\frac{1+v_{1}}{E_{1}}\left[\frac{-R_{1}^{2} R_{2}^{2}\left(p_{c}-p_{i}\right)}{\left(R_{2}^{2}-R_{1}^{2}\right) R}+\frac{\left(1-2 v_{1}\right)\left(R_{1}^{2} p_{i}-R_{2}^{2} p_{c}\right) R}{\left(R_{2}^{2}-R_{1}^{2}\right)}\right], \quad R 1 \leq R \leq R 2 \\
& u_{r r}^{2}(R)=\frac{1+v_{2}}{E_{2}}\left[\frac{-R_{3}^{2} R_{2}^{2}\left(p_{o}-p_{c}\right)}{\left(R_{3}^{2}-R_{2}^{2}\right) R}+\frac{\left(1-2 v_{2}\right)\left(R_{2}^{2} p_{c}-R_{3}^{2} p_{o}\right) R}{\left(R_{3}^{2}-R_{2}^{2}\right)}\right], \quad R 2 \leq R \leq R 3
\end{aligned}
$$

where $\sigma_{\text {rr }}^{1}(\mathrm{R})$ denotes the radial stress in the cylinder 1 at any given radius $R ; \sigma_{\text {rr }}^{2}(R)$ denotes the radial stress in the cylinder 2 at any given radius $\mathrm{R}$. A,B,C and D are the non-zero real unknown constants; $p_{c}$ denotes the radial pressure at the interface; $u_{r r}^{1}(R)$ denotes the radial displacement of the material point at a given radius $\mathrm{R}$ in cylinder 1 and $u_{r r}^{2}(R)$ denotes the radial displacement of the material point at a given radius $\mathrm{R}$ in the cylinder $2 ; v_{1}$ denotes the Poisson's ration for the material of cylinder1 and $v_{2}$ denotes the Poisson's ration for material of cylinder2. $E_{1}$ is the Elastic modulus of material of cylinder 1 and $E_{2}$ is the Elastic modulus of the material in cylinder 2.

The unknown constants A,B,C and D in Eq. (2.18) are determined by using the following boundary and traction and displacement continuity conditions at the interface:

$$
\begin{aligned}
& \sigma_{r r}^{1}\left(R_{1}\right)=-p_{i} \\
& \sigma_{r r}^{2}\left(R_{3}\right)=-p_{o} \\
& \sigma_{r r}^{1}\left(R_{2}\right)=\sigma_{r r}^{2}\left(R_{2}\right)=-p_{c} \\
& u_{r r}^{1}\left(R_{2}\right)=u_{r r}^{2}\left(R_{2}\right)
\end{aligned}
$$

On determination of the above constants using the Eqs. (2.18) the radial stress developed at the interface is

$$
p_{c}=\left[\frac{\left(2\left(1-v_{1}^{2}\right) R_{1}^{2}\left(R_{3}^{2}-R_{2}^{2}\right) E_{2} p_{i}\right)+\left(2\left(1-v_{2}^{2}\right) R_{3}^{2}\left(R_{2}^{2}-R_{1}^{2}\right) E_{1} p_{o}\right)}{\left(\left(1+v_{2}\right)\left(R_{3}^{2}+\left(1-2 v_{2}\right) R_{2}^{2}\right)\left(R_{2}^{2}-R_{1}^{2}\right) E_{1}\right)+\left(\left(1+v_{1}\right)\left(R_{1}^{2}+\left(1-2 v_{1}\right) R_{2}^{2}\right)\left(R_{3}^{2}-R_{2}^{2}\right) E_{2}\right)}\right]
$$


Now with the stress at the interface being known the stresses and the radial displacements in both the cylinders are obtained as:

$\sigma_{r r}^{(j)}(R)=\frac{R_{i}^{2} R_{o}^{2}\left(P_{o}-P_{i}\right)}{\left(R_{o}^{2}-R_{i}^{2}\right) R^{2}}+\frac{R_{i}^{2} P_{i}-R_{o}^{2} P_{o}}{\left(R_{o}^{2}-R_{i}^{2}\right)}$

$\sigma_{\theta \theta}^{(j)}(R)=\frac{-R_{i}^{2} R_{o}^{2}\left(P_{o}-P_{i}\right)}{\left(R_{o}^{2}-R_{i}^{2}\right) R^{2}}+\frac{R_{i}^{2} P_{i}-R_{o}^{2} P_{o}}{\left(R_{o}^{2}-R_{i}^{2}\right)}$

$u_{r r}^{(j)}(R)=\frac{1+v_{j}}{E_{j}}\left[\frac{-R_{i}^{2} R_{o}^{2}\left(P_{o}-P_{i}\right)}{\left(R_{o}^{2}-R_{i}^{2}\right) R}+\frac{\left(1-2 v_{i}\right)\left(R_{i}^{2} P_{i}-R_{o}^{2} P_{o}\right) R}{\left(R_{o}^{2}-R_{i}^{2}\right)}\right]$

where $\mathrm{j}=1$ corresponds to the cylinder 1 for which

$R_{i}=R_{1}, R_{o}=R_{2}, P_{i}=p_{i}, P_{o}=p_{c}, v_{j}=v_{l}$ and $E_{j}=E_{1}$

and $j=2$ corresponds to the cylinder 2 for

$R_{i}=R_{2}, R_{o}=R_{3}, P_{i}=p_{c}, P_{o}=p_{o}, v_{j}=v_{2}$ and $E_{j}=E_{2}$

As discussed earlier these elasticity solutions can be converted to the Laplace Transform of the solution of the problem for the viscoelastic material by making the following substitutions:

Material Properties:

$E \rightarrow a \bar{G}(a)=\frac{1}{a \bar{D}(a)}$

Where $G$ denotes the stress relaxation modulus of the viscoelastic material and $D$ denotes the creep compliance of the viscoelastic material; a is the Laplace Transform parameter. In this study the Poisson's ratio of the viscoelastic material is assumed to be time independent function. However, the Poisson's ratio for viscoelastic materials is a function of time and also the form of time dependence may vary with the type of test like 
relaxation or creep tests. If the Poisson's ratio is taken to be time dependent material function then the analytical answer will have multiple integral involved which is not very easy to solve. Hence in this study time independent function has been assumed.

Field variables:

$$
\begin{aligned}
& \sigma_{r r}^{(j)}(R) \rightarrow \bar{\sigma}_{r r}^{(j)}(R, a) \\
& \sigma_{\theta \theta}^{(j)}(R) \rightarrow \bar{\sigma}_{\theta \theta}^{(j)}(R, a) \\
& u_{r r}^{(j)}(R) \rightarrow \bar{u}_{r r}^{(j)}(R, a)
\end{aligned}
$$

Boundary equations:

$$
\begin{aligned}
& P_{i}(t) \rightarrow \bar{P}_{i}(a) \\
& P_{o}(t) \rightarrow \bar{P}_{o}(a)
\end{aligned}
$$

On substituting Eqs. (2.22)-(2.24) into the Eq. (2.21), the Laplace transformed stress and displacement fields are:

$$
\begin{aligned}
& \bar{\sigma}_{r r}^{(j)}(R, a)=\frac{R_{i}^{2} R_{o}^{2}\left(\bar{P}_{o}-\bar{P}_{i}\right)}{\left(R_{o}^{2}-R_{i}^{2}\right) R^{2}}+\frac{R_{i}^{2} \bar{P}_{i}-R_{o}^{2} \bar{P}_{o}}{\left(R_{o}^{2}-R_{i}^{2}\right)} \\
& \bar{\sigma}_{\theta \theta}^{(j)}(R, a)=\frac{-R_{i}^{2} R_{o}^{2}\left(\bar{P}_{o}-\bar{P}_{i}\right)}{\left(R_{o}^{2}-R_{i}^{2}\right) R^{2}}+\frac{R_{i}^{2} \bar{P}_{i}-R_{o}^{2} \bar{P}_{o}}{\left(R_{o}^{2}-R_{i}^{2}\right)} \\
& \bar{u}_{r r}^{(j)}(R, a)=\left(1+v_{j}\right)\left[\frac{-R_{i}^{2} R_{o}^{2}\left(\bar{P}_{o}(a)-\bar{P}_{i}(a)\right)}{\left(R_{o}^{2}-R_{i}^{2}\right) R}+\frac{\left(1-2 v_{i}\right)\left(R_{i}^{2} \bar{P}_{i}(a)-R_{o}^{2} \bar{P}_{o}(a)\right) R}{\left(R_{o}^{2}-R_{i}^{2}\right)}\right] a \bar{D}_{j}(a)
\end{aligned}
$$

The Eq. (2.25) is inverted to get the final solution for the composite cylinder made up of linear viscoelastic material having time independent Poisson's ratio as:

$$
\sigma_{r r}^{(j)}(R, t)=\frac{R_{i}^{2} R_{o}^{2}\left(P_{o}(t)-P_{i}(t)\right)}{\left(R_{o}^{2}-R_{i}^{2}\right) R^{2}}+\frac{R_{i}^{2} P_{i}(t)-R_{o}^{2} P_{o}(t)}{\left(R_{o}^{2}-R_{i}^{2}\right)}
$$




$$
\begin{aligned}
& \sigma_{\theta \theta}^{(j)}(R, t)=\frac{-R_{i}^{2} R_{o}^{2}\left(P_{o}(t)-P_{i}(t)\right)}{\left(R_{o}^{2}-R_{i}^{2}\right) R^{2}}+\frac{R_{i}^{2} P_{i}(t)-R_{o}^{2} P_{o}(t)}{\left(R_{o}^{2}-R_{i}^{2}\right)} \\
& u_{r r}^{(j)}(R, t)=\left(1+v_{j}\right)\left[\frac{-R_{i}^{2} R_{o}^{2}\left(P_{o}(t)-P_{i}(t)\right)}{\left(R_{o}^{2}-R_{i}^{2}\right) R}+\frac{\left(1-2 v_{i}\right)\left(R_{i}^{2} P_{i}(t)-R_{o}{ }^{2} P_{o}(t) R\right.}{\left(R_{o}^{2}-R_{i}^{2}\right)}\right] * d D_{j}
\end{aligned}
$$

Where the $P_{o} * d D$ denotes the Stieltjes convolution of $P_{o}(t)$ and $D(t)$ defined by

$P_{o} * d D=P_{o}(0) D(t)+\int_{0}^{t} D(t-s) \dot{P}_{o}(s) d s$ and

$P_{i}^{*} d D$ denotes the Stieltjes convolution of $P_{i}(t)$ and $D(t)$ defined by

$$
P_{i}^{*} d D=P_{i}(0) D(t)+\int_{0}^{t} D(t-s) \dot{P}_{i}(s) d s
$$

The radial strain and hoop strain are given by:

$$
\begin{aligned}
& \varepsilon_{r r}^{(j)}(R, t)=\frac{\partial u_{r r}^{(j)}}{\partial R}=\left(1+v_{j}\right)\left[\frac{R_{i}^{2} R_{o}^{2}\left(P_{o}(t)-P_{i}(t)\right)}{\left(R_{o}^{2}-R_{i}^{2}\right) R^{2}}+\frac{\left(1-2 v_{i}\right)\left(R_{i}^{2} P_{i}(t)-R_{o}^{2} P_{o}(t)\right.}{\left(R_{o}^{2}-R_{i}^{2}\right)}\right] * d D_{j}(t) \\
& \varepsilon_{\theta \theta}^{(j)}(R, t)=\frac{u_{r r}^{(j)}(R, t)}{R}=\left(1+v_{j}\right)\left[\frac{-R_{i}^{2} R_{o}^{2}\left(P_{o}(t)-P_{i}(t)\right)}{\left(R_{o}^{2}-R_{i}^{2}\right) R^{2}}+\frac{\left(1-2 v_{i}\right)\left(R_{i}^{2} P_{i}(t)-R_{o}^{2} P_{o}(t)\right.}{\left(R_{o}^{2}-R_{i}^{2}\right)}\right] * d D_{j}(t)
\end{aligned}
$$

\subsection{TEMPERATURE DEPENDENT RESPONSE OF VISCOELASTIC COMPOSITE CYLINDER}

The response of viscoelastic materials is strongly dependent on the temperature the material is subjected to. Schwarzl and Staverman [9] introduced a terminology of Thermorheologically Simple Material (TSM) where change in the viscoelastic material behavior due to temperature is completely equivalent to a corresponding shift of the logarithmic time scale. The time-temperature superposition proposed by Leaderman[8] 
is applicable to describe the temperature dependent viscoelastic behavior of TSM. Morland and Lee [10] and Rajagopal and Wineman [30] discussed the extension of timetemperature superposition principle applicable to TSM to time-varying temperature histories. In summary, materials can be categorized as TSM when they exhibit the following features:

1. The shape of stress relaxation/creep compliance curves on the $\log t$ scale at different temperatures is same.

2. The initial and the long time values of the relaxation/creep compliance modulus are independent of temperature.

3. During the creep or stress relaxation essentially the same macromolecular reconfigurations takes place at the speed that is temperature dependent. ( Higher temperature increases the speed and lower temperatures reduces the speed of molecular events)

Time-temperature superposition is applicable to TSM that describes viscoelastic behavior with respect to time and temperature. It implies that stress relaxation/creep compliance at any temperature can be expressed by two terms: relaxation/creep compliance at a reference temperature and a function known as shift function (which is a material property that denotes the amount of time shifting to be done on the stress relaxation vs $\log$ time graph). Using this time-temperature equivalence a relation is developed to evaluate stress relaxation at any temperature $\mathrm{T}$ from the known value of stress relaxation at a reference temperature $T_{0}$ : 
$G[t, \stackrel{t}{t(s)}]=G\left[\int_{0=0}^{t} \frac{d s}{a\left(T(s), T_{o}\right)}, T_{o}\right]$

where the integral $\xi(t)=\int_{0}^{t} \frac{d t}{a\left(T(t), T_{o}\right)}$ is known as 'reduced time' (Morland and Lee

[10] ) and the function $a\left(T(t), T_{o}\right)$ is the shift function which is a material property.

When the temperature dependence on the material properties of the composite cylinder is modeled as TSM, the stress and displacement fields with time varying temperature represented by Eq. (2.27) have the following form:

$$
\begin{aligned}
& \sigma_{r r}^{(j)}(R, t)=\frac{R_{i}^{2} R_{o}^{2}\left(P_{o}(\xi(t))-P_{i}(\xi(t))\right)}{\left(R_{o}^{2}-R_{i}^{2}\right) R^{2}}+\frac{R_{i}^{2} P_{i}(\xi(t))-R_{o}^{2} P_{o}(\xi(t))}{\left(R_{o}^{2}-R_{i}^{2}\right)} \\
& \sigma_{\theta \theta}^{(j)}(R, t)=\frac{-R_{i}^{2} R_{o}^{2}\left(P_{o}(\xi(t))-P_{i}(\xi(t))\right)}{\left(R_{o}^{2}-R_{i}^{2}\right) R^{2}}+\frac{R_{i}^{2} P_{i}(\xi(t))-R_{o}^{2} P_{o}(\xi(t))}{\left(R_{o}^{2}-R_{i}^{2}\right)} \\
& u_{r r}^{(j)}(R, t)=\left(1+v_{j}\right)\left[\frac{-R_{i}^{2} R_{o}^{2}\left(P_{o}(\xi(t))-P_{i}(\xi(t))\right)}{\left(R_{o}^{2}-R_{i}^{2}\right) R}+\frac{\left(1-2 v_{j}\right)\left(R_{i}^{2} P(\xi(t))_{i}-R_{o}^{2} P_{o}(\xi(t)) R\right.}{\left(R_{o}^{2}-R_{i}^{2}\right)}\right] * d D_{j}
\end{aligned}
$$

Where

$$
\begin{aligned}
& P_{o}^{*} d D=P_{o}(0) D(\xi(t))+\int_{0}^{t} D(\xi(t)-\xi(s)) d P_{o}(s) \\
& P_{i} * d D=P_{i}(0) D(\xi(t))+\int_{0}^{t} D(\xi(t)-\xi(s)) d P_{i}(s)
\end{aligned}
$$

A class of materials that exhibit more general thermo-viscoelastic behaviors are attributed as TCM. The temperature has strong influence on the initial and long term properties of the material. The effect of temperature dependence on the viscoelastic behavior of the composite cylinder is studied as TCM so as to study the significant effect of temperature on the overall response. To this end the transient tensile creep 
compliance of the material of two layers are chosen to have Prony series representation which consists of series of exponential forms. The elastic and the transient parts are both made to be temperature dependent along with temperature dependent shift factor. It is assumed that all these material creep properties at a particular location depend on the instantaneous value of the temperature at a given location at a particular time and not the entire history of the temperature. So creep compliance of the material has the form:

$D(t, T)=D_{0}(T)+\sum_{n=1}^{n} D_{n}(T)\left[1-\exp \left(-\lambda_{n} \xi(t)\right)\right]$

where $\xi(t)=\int_{0}^{t} \frac{d s}{a\left(T, T_{o}\right)}$

Where $D_{0}$ denotes the elastic part; $D_{n}$ denotes the coefficient of the $\mathrm{n}^{\text {th }}$ term in the series of transient creep; $\lambda_{n}$ denotes the reciprocal of the characteristic of creep time; $\xi(t)$ is the reduced time and $\mathrm{a}\left(\mathrm{T}, \mathrm{T}_{\mathrm{o}}\right)$ is the shift factor.

To get the deformation and stress fields, the temperature value at a time instant and a given material point is obtained from solving the heat conduction equation. The temperature dependent creep compliance in Eq. (2.30) is then calculated together with the reduced time in Eq. (2.31). The Eqs. (2.30) and (2.31) are substituted to the Eq. (2.29) to obtain the stress, displacement and strain fields of the viscoelastic composite cylinder. 


\section{CHAPTER III}

\section{ANALYTICAL AND NUMERICAL SOLUTION FOR THERMO- MECHANICAL RESPONSE OF THE COMPOSITE CYLINDER}

The first section of this chapter describes the geometry, material parameters and the mechanical loading conditions to which the composite cylinder is subjected. The analytical and the FE solutions for the stress, strain and displacement fields are discussed. FE software ABAQUS is used to obtain the solution for the heat conduction and the deformation of the composite cylinder. The convergence study is carried out to ensure that the time increment and the FE mesh gives numerical results within adequate accuracy. In the final section, the analytical and numerical solutions for the stress, strain and displacement fields are compared.

\subsection{ANALYTICAL SOLUTION OF THE FIELD VARIABLES FOR THE VISCOELASTIC COMPOSITE CYLINDER}

The composite cylinder consisting of two layers of linear viscoelastic materials is considered which is assumed to be infinitely long and thus the plain strain conditions are imposed while predicting the mechanical response. The layer 1 is considered to have inner most and outer most radius of 1 and 2 units respectively and the layer 2 is considered to have inner most and outer most radius 2 and 3 units respectively. The composite is assumed to have sufficiently strong bonding at the interface at $r=2$. The non-dimensionalized material properties and loads (thermal and mechanical loads) are used. The two layers are assumed to be isotropic and the tensile and compressive 
properties are identical. The values of material properties of the two layers are as described in Table 3.1.

Table 3.1. Elastic properties of the material of two layers

\begin{tabular}{|c|c|c|c|}
\hline Parameter & Value & Parameter & Value \\
\hline$v^{(1)}$ & 0.25 & $v^{(2)}$ & 0.29 \\
\hline$K^{(1)}$ & 1 & $K^{(2)}$ & 1.5 \\
\hline$E_{0}^{(1)}$ & 5.128 & $E_{0}^{(2)}$ & 4.0 \\
\hline
\end{tabular}

where $v^{(i)}$ denote the Poisson's ratio for $i^{\text {th }}$ layer; where $i=1,2$ corresponds to two layers. $\mathrm{K}^{(\mathrm{i})}$ denotes the thermal conductivity for the $\mathrm{i}^{\text {th }}$ layer and $\mathrm{E}_{0}{ }^{(\mathrm{i})}$ denotes the elastic (instantaneous) part of compliance of the $\mathrm{i}^{\text {th }}$ layer. The transient creep compliance of the materials in the two layers are represented by Prony series, as mentioned in chapter II. The non-dimensional elastic compliance and transient creep properties of the material of the two layers are shown in Table 3.2 where $n$ is the number of Prony terms, $D_{n}$ is the $n^{\text {th }}$ Prony coefficient, $\lambda_{\mathrm{n}}$ is the reciprocal of the characteristic of the $\mathrm{n}^{\text {th }}$ retardation time and $\mathrm{D}_{0}$ is the elastic compliance.

Now using the Laplace Transform method discussed by Rajagopal and Wineman [6], from these creep compliances, the stress relaxation moduli of the layer 1 and layer 2 denoted by $\mathrm{G}_{1}(\mathrm{t})$ and $\mathrm{G}_{2}(\mathrm{t})$ respectively, are:

$$
\begin{aligned}
& G_{1}(t)=2.105+1.36 e^{-1.36 t}+e^{-0.0135 t}+0.66 e^{-0.0013 t} \\
& G_{2}(t)=2.632+0.155 e^{-1.04 t}+0.64 e^{-0.012 t}+0.57 e^{-0.0012 t}
\end{aligned}
$$


Table 3.2. Elastic compliance and transient properties of the creep compliance for the material of two layers

\begin{tabular}{|c|c|c|c|c|c|}
\hline \multicolumn{3}{|c|}{ Layer 1 } & \multicolumn{3}{c|}{ Layer 2 } \\
\hline $\mathrm{n}$ & $\lambda_{\mathrm{n}}$ & $\mathrm{D}_{\mathrm{n}}$ & $\mathrm{n}$ & $\lambda_{\mathrm{n}}$ & $\mathrm{D}_{\mathrm{n}}$ \\
\hline 1 & 1 & 0.07 & 1 & 1 & 0.01 \\
2 & 0.01 & 0.09 & 2 & 0.01 & 0.05 \\
3 & 0.001 & 0.12 & 3 & 0.001 & 0.07 \\
\hline \multicolumn{3}{|c|}{$\mathrm{D}_{0}=0.195$} & \multicolumn{3}{c|}{$\mathrm{D}_{0}=0.25$} \\
\hline
\end{tabular}

On substituting the above values of material properties and the dimensions of the cylinder considered in Eq. (2.20) and taking the Laplace transform, the Laplace transformed stress at the interface becomes:

$\bar{p}_{c}(a)=\frac{9.375 \bar{G}_{2}(a) \bar{p}_{i}(a)+49.46 \bar{G}_{1}(a) \bar{p}_{o}(a)}{41.33 \bar{G}_{1}(a)+18.75 \bar{G}_{2}(a)}$

where $p_{i}$ denotes the pressure on the innermost radius of $r=1.0$ and $p_{o}$ denotes the pressure on the outermost radius of $\mathrm{r}=3.0$ and $\mathrm{a}$ is the Laplace Transform variable. The stress at the interface is then obtained by taking the Laplace inverse of the Eq. (3.3).

The stress, displacement and strain fields in the layer 1 of the composite cylinder are obtained by substituting the material properties and radii values of layer 1 in Eq. (2.26) and (2.27), which for temperature independent material properties are:

$$
\begin{aligned}
& \sigma_{r r}^{(1)}(R, t)=\frac{1}{3}\left[\left(\frac{4}{R^{2}}-4\right) p_{c}(t)+\left(\frac{-4}{R^{2}}+1\right) p_{i}(t)\right], \forall t \geq 0 \\
& \sigma_{\theta \theta}^{(1)}(R, t)=\frac{1}{3}\left[\left(\frac{-4}{R^{2}}-4\right) p_{c}(t)+\left(\frac{4}{R^{2}}+1\right) p_{i}(t)\right], \forall t \geq 0
\end{aligned}
$$




$$
\begin{aligned}
& u_{r r}^{(1)}(R, t)=\frac{1.25}{3}\left[\left(\frac{-4}{R}-2 R\right) p_{c}(t)+\left(\frac{4}{R}+0.5 R\right) p_{i}\right] * d D_{1}(t), \forall t \geq 0 \\
& \varepsilon_{r r}^{(1)}(R, t)=\frac{1.25}{3}\left[\left(\frac{4}{R^{2}}-2\right) p_{c}(t)+\left(\frac{-4}{R^{2}}+0.5\right) p_{i}(t)\right] * d D_{1}(t), \forall t \geq 0 \\
& \varepsilon_{\theta \theta}^{(1)}(R, t)=\frac{1.25}{3}\left[\left(\frac{-4}{R^{2}}-2\right) p_{c}(t)+\left(\frac{4}{R^{2}}+0.5\right) p_{i}\right] * d D_{1}(t), \forall t \geq 0
\end{aligned}
$$

Similarly, on substituting the material properties and radii values of layer 2 in the Eqs. (2.26) and (2.27), the stress, displacement and strain fields for the layer 2 are:

$$
\begin{aligned}
& \varepsilon_{\theta \theta}^{(1)}(R, t)=\frac{1.25}{3}\left[\left(\frac{-4}{R^{2}}-2\right) p_{c}(t)+\left(\frac{4}{R^{2}}+0.5\right) p_{i}\right] * d D_{1}(t), \forall t \geq 00 \\
& \varepsilon_{\theta \theta}^{(2)}(R, t)=\frac{1.29}{5}\left[\left(\frac{-36}{R^{2}}-3.78\right) p_{c}(t)+\left(\frac{36}{R^{2}}+1.68\right) p_{i}\right] * d D_{2}(t), \forall t \geq 0 \\
& \sigma_{r r}^{(2)}(R, t)=\frac{1}{5}\left[\left(\frac{36}{R^{2}}-9\right) p_{c}(t)+\left(\frac{-36}{R^{2}}+4\right) p_{i}(t)\right], \forall t \geq 0 \\
& \sigma_{\theta \theta}^{(2)}(R, t)=\frac{1}{5}\left[\left(\frac{-36}{R^{2}}-9\right) p_{c}(t)+\left(\frac{36}{R^{2}}+4\right) p_{i}(t)\right], \forall t \geq 0 \\
& u_{r r}^{(2)}(R, t)=\frac{1.29}{5}\left[\left(\frac{-36}{R}-3.78 R\right) p_{c}(t)+\left(\frac{36}{R}+1.68 R\right) p_{i}\right] * d D_{2}(t), \forall t \geq 0 \\
& \varepsilon_{r r}^{(2)}(R, t)=\frac{1.29}{5}\left[\left(\frac{36}{R^{2}}-3.78\right) p_{c}(t)+\left(\frac{-36}{R^{2}}+1.68\right) p_{i}(t)\right] * d D_{2}(t), \forall t \geq 0 \\
& \varepsilon_{\theta \theta}^{(2)}(R, t)=\frac{1.29}{5}\left[\left(\frac{-36}{R^{2}}-3.78\right) p_{c}(t)+\left(\frac{36}{R^{2}}+1.68\right) p_{i}\right] * d D_{2}(t), \forall t \geq 0
\end{aligned}
$$

As discussed in chapter II, the effect of temperature on the overall viscoelastic response of the composite cylinder is studied by considering the material properties at a given location to be dependent on temperature at that location. In this study we have 
assumed the material properties of elastic compliance $\left(D_{0}\right)$, transient creep compliance $\left(D_{n}\right)$ and time shift factor $(a(T))$ to depend on temperature. $D_{o}$ and $D_{n}$ are assumed to linearly depend on the temperature. We could also have considered the variation of polynomial or exponential form. The specific form of temperature dependence is chosen to merely illustrate that the effect of temperature on the stress, strain and displacement fields can be pronounced. The magnitude of strain developed will definitely depend on the form of temperature dependence assumed.

The linear temperature dependence assumed in this study is:

$D_{o}^{(i)}(T)=D_{o}^{(i)}(1+T)$

$D_{n}^{(i)}(T, t)=D_{n}^{(i)}(0.5 T)\left(1-e^{\left(-\lambda_{n} \xi(t)\right)}\right) \quad \mathrm{i}=1,2$ corresponds to layers 1 and 2

where $\xi(t)=\int_{0}^{t} \frac{d s}{a\left(T(s), T_{o}\right)}$ is the reduced time with

$a\left(T, T_{o}\right)=\frac{1}{1+\left(T-T_{o}\right)}$

where $T_{0}$ is the reference temperature considered to be 0 in this study since non dimensional quantities are considered in this study. The temperature dependent material properties given by Eqs. (3.6) and (3.7) are substituted in Eq. (2.30) which is then substituted in Eq. (2.29) to obtain the stress, displacement and strain fields for a composite cylinder with temperature dependent material properties. 


\subsection{FINITE ELEMENT SOLUTION FOR THE SEQUENTIALLY COUPLED HEAT CONDUCTION AND DEFORMATION IN THE VISCOELASTIC COMPOSITE CYLINDER}

The numerical approach is used to solve the composite cylinder problem, as it is not always possible to obtain the closed form analytical solution when the complex geometry with more general thermo-mechanical loadings is considered. The Finite Element (FE) mesh wherein the governing equations for the conduction of heat and displacement of composite cylinder are solved, is generated using three-dimensional continuum quadratic elements of the ABAQUS FE software. One quarter symmetric FE mesh is shown in the Fig. 3.1. The boundary conditions used in the FE mesh are: $u_{x}$ $($ Face 1$)=0.0, \mathrm{u}_{\mathrm{y}}($ Face 2$)=0.0$ and for imposing the plane strain conditions for infinitely long annular cylinder $\mathrm{u}_{\mathrm{z}}($ Face 3$)=\mathrm{u}_{\mathrm{z}}($ Face 4$)=0.0$.

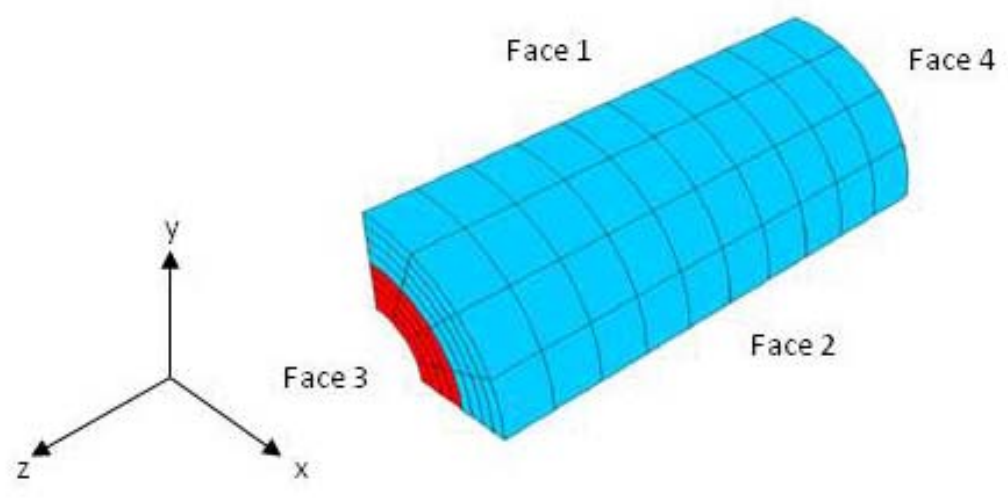

Fig. 3.1. FE mesh for composite cylinder 
The solution of the coupled displacement-heat conduction equations are performed in two sequential steps. Firstly the heat transfer analysis is carried out where the temperature profile of the body is evaluated. Then by using these temperature values, the material properties are made temperature dependent as shown in Eqs. (3.6) and (3.7) and the stress and deformation fields are evaluated. To incorporate the effect of temperature on the deformation of the viscoelastic composite cylinder, the constitutive model incorporating time and temperature effects is numerically solved and implemented in the material subroutine (UMAT) of the ABAQUS FE code. The numerical algorithm developed by Haj-Ali and Muliana [29] for the stress dependent viscoelastic material model that is compatible with displacement based FE framework is modified to incorporate the temperature effects. This algorithm is used to predict the time and temperature dependent viscoelastic material response under general mechanical and thermal loading histories. The linearized solution within an incremental time step is first obtained. Iterative scheme is used to obtain solutions of nonlinear problems at structural and material levels. As shown in Eq. (3.1) at each iteration within the incremental time-step $\Delta \mathrm{t}$, trial incremental strain tensor $\Delta \varepsilon_{i j}^{t}$ and temperature $\Delta \mathrm{T}^{\mathrm{t}}$ are obtained. The goal is to calculate the current total stresses $\Delta \sigma_{i j}^{t}$ and the material consistent tangent stiffness $C_{i j k l}^{t}$ from the given current variables and the total stress and strain history variables stored from the previous converged solution at time $(\mathrm{t}-\Delta \mathrm{t})$. The converged $C_{i j k l}^{t}$ at the current time $\mathrm{t}$ is used to provide incremental trial strains for the next time step $(\mathrm{t}+\Delta \mathrm{t})$. 
Given : $\Delta \varepsilon_{i j}^{t}, \Delta T^{t}, \Delta t$

$$
\begin{gathered}
\varepsilon_{i j}^{t}=\varepsilon_{i j}^{t-\Delta t}+\Delta \varepsilon_{i j}^{t} \\
\mathrm{~T}^{t}=T^{t-\Delta t}+\Delta T^{t} \\
t=(t-\Delta t)+\Delta t
\end{gathered}
$$

Calculate: Incremental form

$$
\begin{aligned}
\sigma_{i j}^{t} & =\sigma_{i j}^{t-\Delta t}+\Delta \sigma_{i j}^{t} \\
\Delta \sigma_{i j}^{t} & =f\left(T^{t}, \Delta \varepsilon_{i j}^{t}\right) \\
C_{i j k l}^{t} & =\frac{\partial \Delta \sigma_{i j}^{t}}{\partial \Delta \varepsilon_{i j}^{t}}
\end{aligned}
$$

or Total form

$$
\begin{aligned}
\sigma_{i j}^{t} & =f\left(c^{t}, \varepsilon_{i j}^{t}\right) \\
C_{i j k l}^{t} & =\frac{\partial \sigma_{i j}^{t}}{\partial \varepsilon_{i j}^{t}}
\end{aligned}
$$

A general single integral relation is used for a linear viscoelastic material and is modified to incorporate the temperature effects, which is written as:

$$
\varepsilon^{t} \equiv \varepsilon(t)=g_{0}\left(T^{t}\right) D_{0} \sigma^{t}+g_{1}\left(T^{t}\right) \int_{0}^{t} \Delta D\left(\psi^{t}-\psi^{\tau}\right) \frac{d \sigma^{\tau}}{d \tau} d \tau
$$

where $D_{0}$ is the uniaxial instantaneous elastic compliance and $\Delta D$ is the transient creep compliance, $\psi$ is the reduced time (effective time) defined by:

$$
\psi^{t} \equiv \psi(t)=\int_{0}^{t} \frac{d \xi}{a\left(T^{\xi}\right)}
$$

The function $\mathrm{a}(\mathrm{T})$ is a temperature dependent material property used to define the time scale shift factor for Thermorheologically Simple Materials. Here the parameter $\mathrm{g}_{0}$ measures the increase or decrease in the instantaneous compliance with the change in 
temperature and the parameter $g_{1}$ measures the increase or decrease in the transient creep compliance with change in temperature.

This uniaxial viscoelastic relation in Eq. (3.2) is generalized for multiaxial (3D) constitutive relations of the isotropic materials by separating the deviatoric and volumetric relations as:

$$
\begin{aligned}
& \varepsilon_{i j}^{t}=e_{i j}^{t}+\frac{1}{3} \varepsilon_{k k}^{t} \delta_{i j}^{t} \\
& e_{i j}^{t}=\frac{1}{2} g_{0}\left(T^{t}\right) J_{0} S_{i j}^{t}+\frac{1}{2} g_{1}\left(T^{t}\right) \int_{0}^{t} \Delta J\left(\psi^{t}-\psi^{\tau}\right) \frac{d S_{i j}^{\tau}}{d \tau} d \tau \\
& \varepsilon_{k k}^{t}=\frac{1}{3} g_{0}\left(T^{t}\right) B_{0} \sigma_{k k}^{t}+\frac{1}{3} g_{1}\left(T^{t}\right) \int_{0}^{t} \Delta B\left(\psi^{t}-\psi^{\tau}\right) \frac{d \sigma_{k k}^{\tau}}{d \tau} d \tau
\end{aligned}
$$

where $e_{i j}, \varepsilon_{k k}, S_{i j}$ and $\sigma_{k k}$ are used to denote the deviatoric strain, volumetric strain, deviatoric stress and volumetric stress, respectively. The terms $\mathrm{J}_{0}$ and $\mathrm{B}_{0}$ are the instantaneous elastic shear and bulk compliances, respectively. The terms $\Delta \mathrm{J}$ and $\Delta \mathrm{B}$ are the transient shear and transient bulk compliances respectively. A Prony Series representation that has a series of exponential functions is used to represent the transient part due to the advantage of this representation in solving the integral form in Eq. (3.4) recursively. The material is assumed to have time independent Poisson's ratio $v$ which allows the shear and bulk compliances to be expressed as:

$$
\begin{array}{ll}
J_{0}=2(1+v) D_{0} & B_{0}=3(1-2 v) D_{0} \\
\Delta J^{\psi^{t}}=2(1+v) \Delta D^{\psi^{t}} & \Delta B^{\psi^{t}}=2(1+v) \Delta D^{\psi^{t}}
\end{array}
$$

where uniaxial transient compliance is expressed in terms of Prony series as:

$$
\Delta D^{\psi^{t}}=\sum_{n=1}^{N} D_{n}\left(1-\exp \left[-\lambda_{n} \psi^{t}\right]\right)
$$


Using Eqs. (3.5) and (3.6), the deviatoric and volumetric strains in Eq. (3.4) can be written as:

$e_{i j}^{t}=\frac{1}{2} g_{0}^{t} J_{0} S_{i j}^{t}+\frac{1}{2} g_{1}^{t} S_{i j}^{t} \sum_{n=1}^{N} J_{n}-\frac{1}{2} g_{1}^{t} \sum_{n=1}^{N} J_{n} q_{i j, n}^{t}$

where $q_{i j, n}^{t}=\int_{0}^{t} \exp \left[-\lambda_{n}\left(\psi^{t}-\psi^{\tau}\right)\right] \frac{d S_{i j}^{\tau}}{d \tau} d \tau$

$\varepsilon_{k k}^{t}=\frac{1}{3} g_{0}^{t} B_{0} \sigma_{k k}^{t}+\frac{1}{3} g_{1}^{t} \sigma_{k k}^{t} \sum_{n=1}^{N} B_{n}-\frac{1}{3} g_{1}^{t} \sum_{n=1}^{N} B_{n} q_{k k, n}^{t}$

where $q_{k k, n}^{t}=\int_{0}^{t} \exp \left[-\lambda_{n}\left(\psi^{t}-\psi^{\psi}\right)\right] \frac{d \sigma_{k k}^{\tau}}{d \tau} d \tau$

A recursive integration method, derived by Taylor et al [25] for linear viscoelastic integral model and extended by Haj-Ali and Muliana [29] for nonlinear viscoelastic constitutive model, is used to solve the integral parts in Eqs. (3.7) and (3.8). Thus by using UMAT, time and temperature dependent constitutive model is used and the stress, strain and displacement fields are predicted.

The numerical approach of finite element method is an approximate method of getting the solution of the problem. It is thus imperative to ensure that the finite element model predicts the response of the material within desired accuracy. The parameters like time increment and element size affect the accuracy of the results obtained by finite element approach. In order to ensure that the solution obtained by numerical approximation is within good accuracy, a parametric study is carried out to examine the effect of using different time increment on the overall material response. This is important so as to determine the range of time increment that can be used such that the final solution is agreeable with the analytical solution within acceptable error. The 
initial radial strain values for the material points on the innermost boundary $(r=R 1)$ are obtained for different time-increment sizes and compared with analytical solution as shown in Fig. 3.2. As seen from the Fig. 3.2 large time increment may lead to a divergent solution. It is concluded that an initial time increment can be chosen in the range of $10^{-2}$ to $10^{-6}$ unit time in order to represent the instantaneous response of the material considered here.

Another study is also carried out to examine the effect of mesh refinement on the accuracy of the results obtained. Owing to the symmetry of the problem, one quarter of the cylinder is modeled with innermost radius of 1 unit and outermost radius of 3 units with interface at $\mathrm{r}=2$ units and cylinder length of 1 unit. A 20-node brick element with reduced integration is used for analyses. A total of 12 elements are used through thickness and 20 elements are used along the circumference as shown in the Fig. 3.3.a. The instantaneous material response in terms of radial strain ar $\mathrm{r}=\mathrm{R} 3$ is compared with the analytical solution and is found to be again within $0.5 \%$ error. A coarse mesh is generated by using 8 elements through the thickness and 4 elements along the circumference as shown in Fig. 3.3.b. The instantaneous material response in terms of radial strain at $\mathrm{r}=\mathrm{R} 3$ with initial time increment of $10^{-5}$ units is obtained and compared with the analytical solution and is found to be again within $0.5 \%$ error. It is thus concluded that such a fine mesh is not required as the coarse mesh mentioned later is sufficient enough to predict the instantaneous response within desired accuracy of $0.5 \%$ error. 


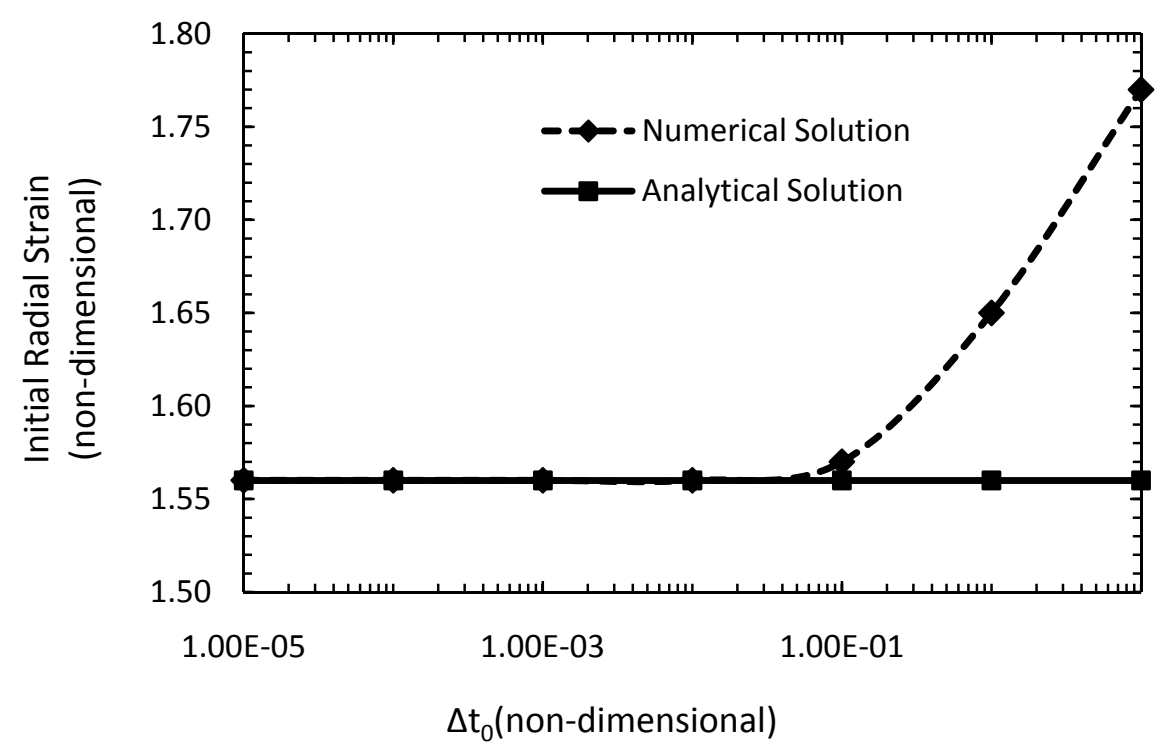

Fig. 3.2. Effect of initial time increment size on the instantaneous static response of the composite cylinder at $\mathrm{r}=1.0$

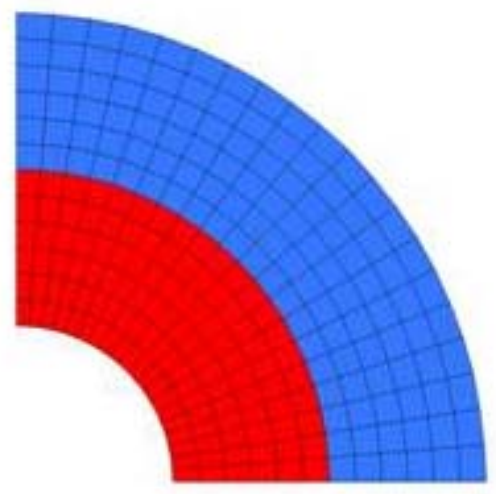

(a)

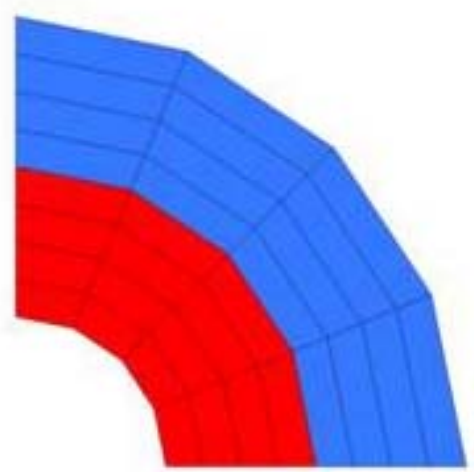

(b)

Fig. 3.3. Finite element mesh convergence study 
A study is also carried out to understand the effect of time increment on the accuracy of the results obtained for the problem of heat conduction. As mentioned earlier, a temperature of 1.0 is imposed on the outermost radius of $r=3.0$ and as a result heat is conducted across the body in radially inward direction. The body is said to have achieved the steady state when the entire body is at the temperature of 1.0. The temperature profile of the body is obtained by carrying out a transient heat transfer analysis in Abaqus FE network till the steady state is achieved. A study is carried out to understand the effect of time increment sizes on the accuracy of the steady state results obtained. Fig. 3.4 shows the plot of temperature versus total time to attain steady state at $\mathrm{r}=1$, from the FE analyses with different time increment of $\mathrm{t}=0.25, \mathrm{t}=0.125, \mathrm{t}=0.05$ and $\mathrm{t}=0.025$. It is concluded that an initial time increment of $\mathrm{t}=0.05$ or lower gives the sufficiently accurate results. In this problem, the steady state is achieved at around $t \approx$ 19. 


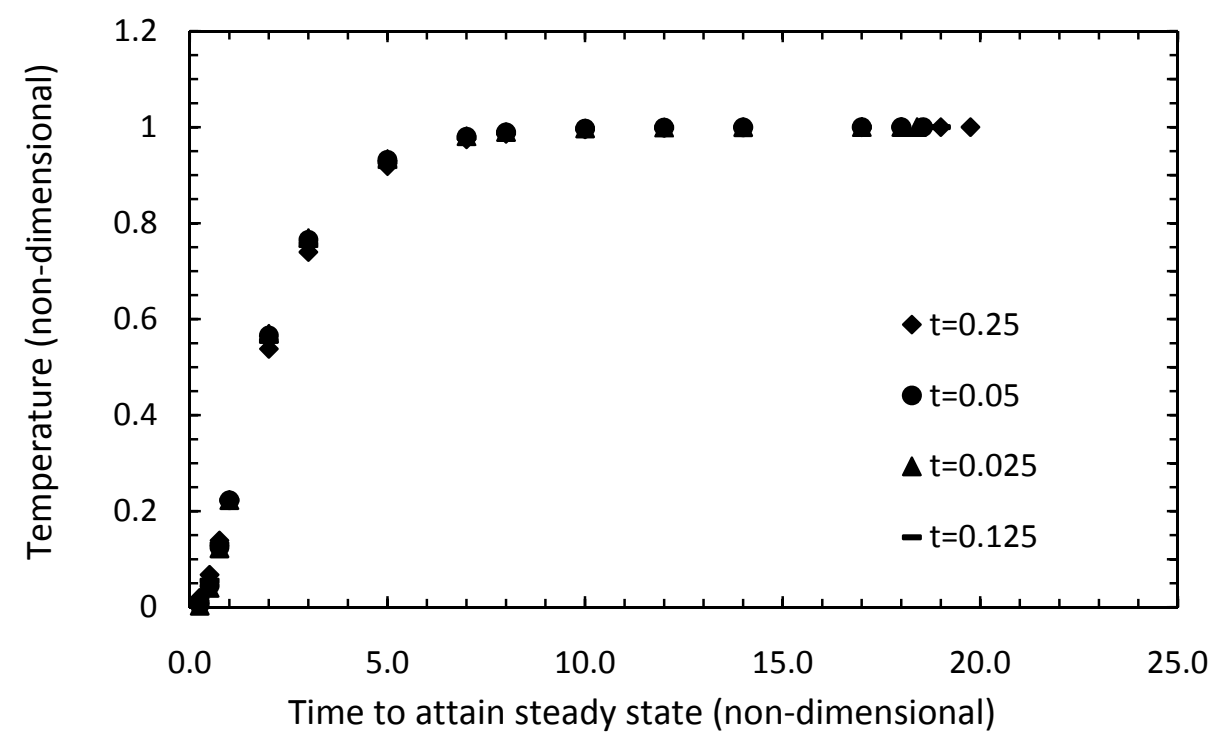

Fig. 3.4. Effect of time increment on the steady state achieved during transient heat transfer at $\mathrm{r}=1.0$ in the composite cylinder

From the parametric studies mentioned above it is ensured that the finite element mesh and the initial time increment are adequate enough to capture the response of the material within good accuracy. Thus this finite element model is further employed to obtain the solution of heat conduction equation and subsequently to obtain the field variables of stress, strain and deformation of the viscoelastic composite cylinder. Transient heat transfer analysis is carried out until the body reaches steady state. The temperature profile is obtained throughout the body by obtaining the temperature values at all the material points for all different times until steady state is achieved. The evolution of temperature field is plotted in Fig. 3.5. This FE model that incorporates the time and temperature effects in the constitutive relations through UMAT is further employed to obtain the solution for the stress, strain and displacement fields. 


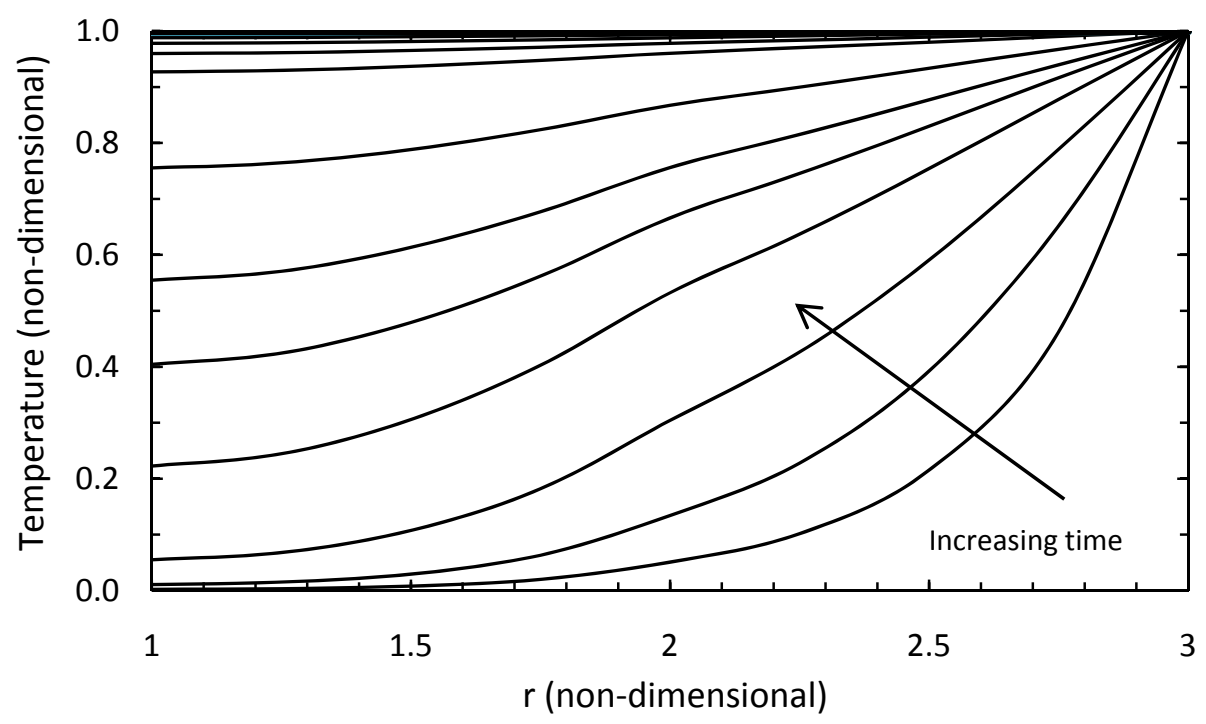

Fig. 3.5. Evolution of temperature across the body due to heat conduction

\subsection{COMPARISON OF ANALYTICAL AND FINITE ELEMENT SOLUTION FOR THE FIELD VARIABLES OF THE VISCOELASTIC COMPOSITE CYLINDER}

The solutions obtained using the analytical and numerical approaches are compared in this section and it is shown that both the results are in good agreement. Two case studies are performed. In the case 1, a composite cylinder made up of two layers of viscoelastic materials whose material properties are independent of temperature is considered with the material parameters as mentioned in the Table 3.1 and 3.2. The inner most boundary of the cylinder at $r=1.0$ is subjected to a uniform pressure of 10.0 and the outer most boundary of the cylinder at $r=3.0$ is subjected to a uniform pressure of 30.0. Using the Eqs. (3.4) and (3.5), the analytical solutions for the stress, displacement 
and the strain fields are obtained for the layer 1 and layer 2. The finite element model discussed in section 3.2 is also used to obtain the solution of the stress, strain and displacement fields for the composite cylinder. The results obtained from the analytical and numerical approaches are compared and found to be having good agreement as the maximum error is within 3-5\% which is acceptable for this study, as shown in the Fig. 3.6 to 3.10 .

In case 2, the effect of temperature on the overall response of the viscoelastic composite cylinder is studied by considering the temperature dependent elastic compliance $\left(D_{0}\right)$ and the transient creep compliance $\left(D_{n}\right)$ and the shift factor $(a(T))$ as mentioned in the Eq. (3.6) and (3.7). The results obtained from the analytical and finite element approaches are within the acceptable error of 3-5\% as seen in the Fig. 3.11 to 3.15 .

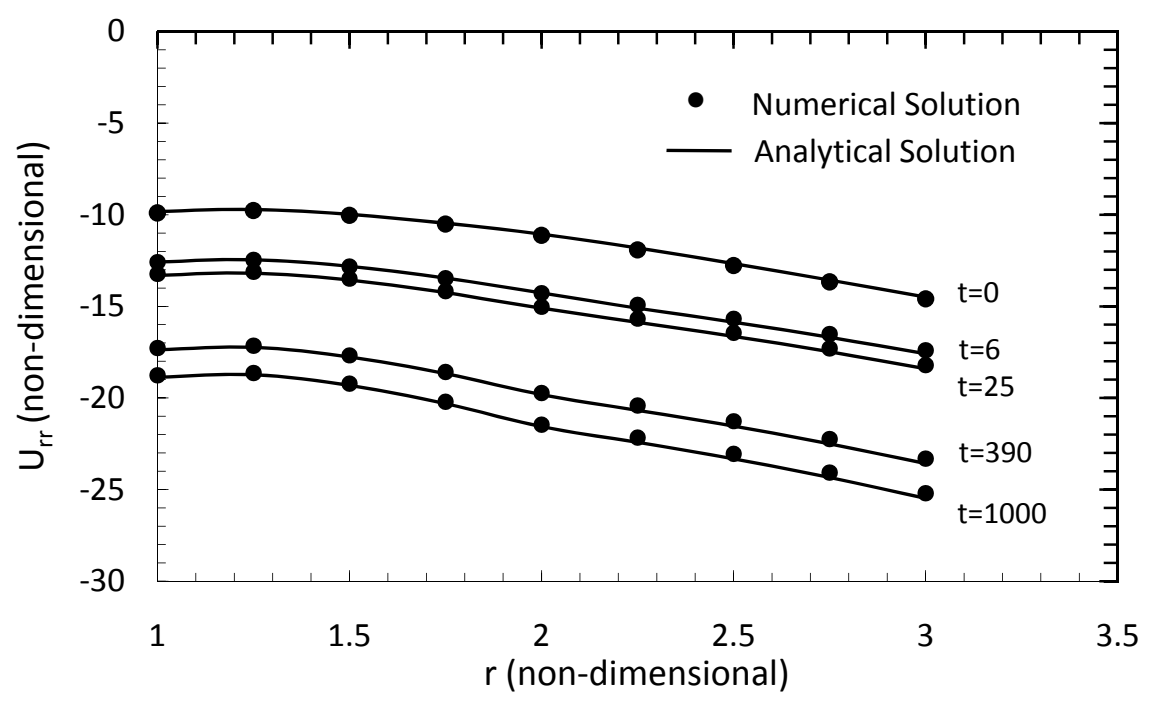

Fig. 3.6. Radial displacement in the composite cylinder as a function of time 


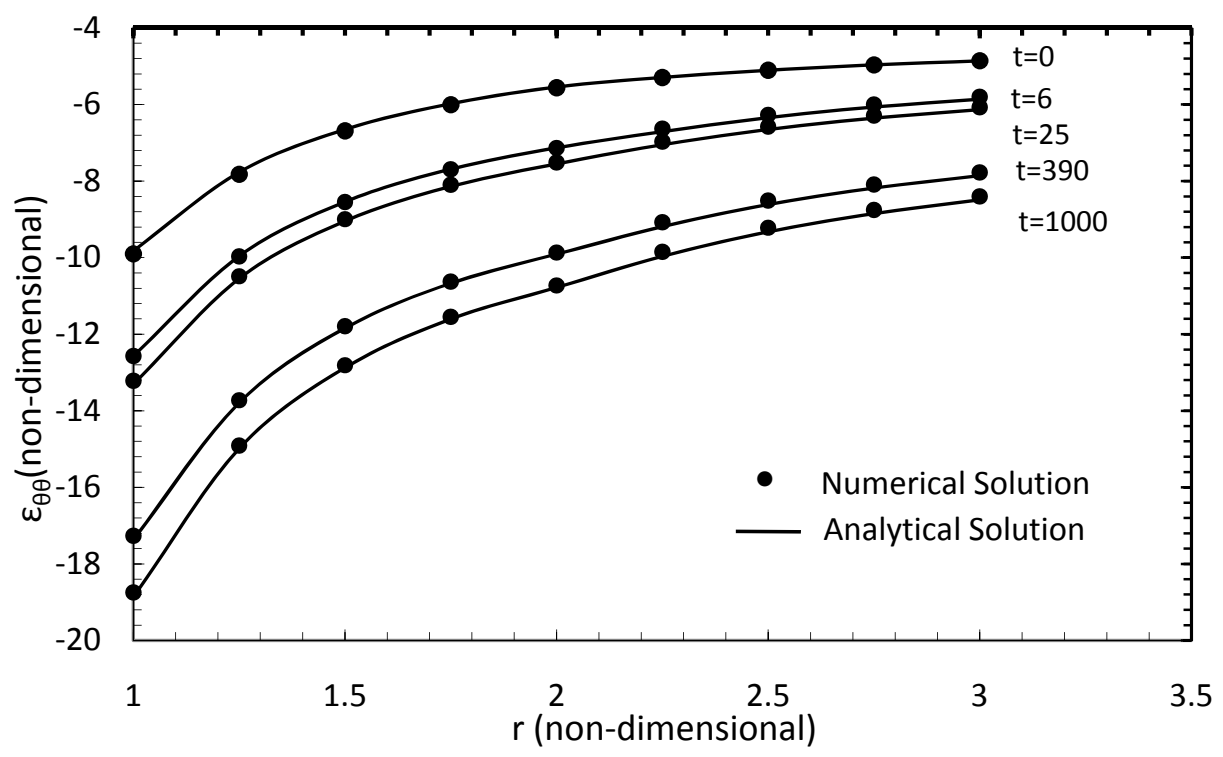

Fig. 3.7. Hoop strain in the composite cylinder as a function of time

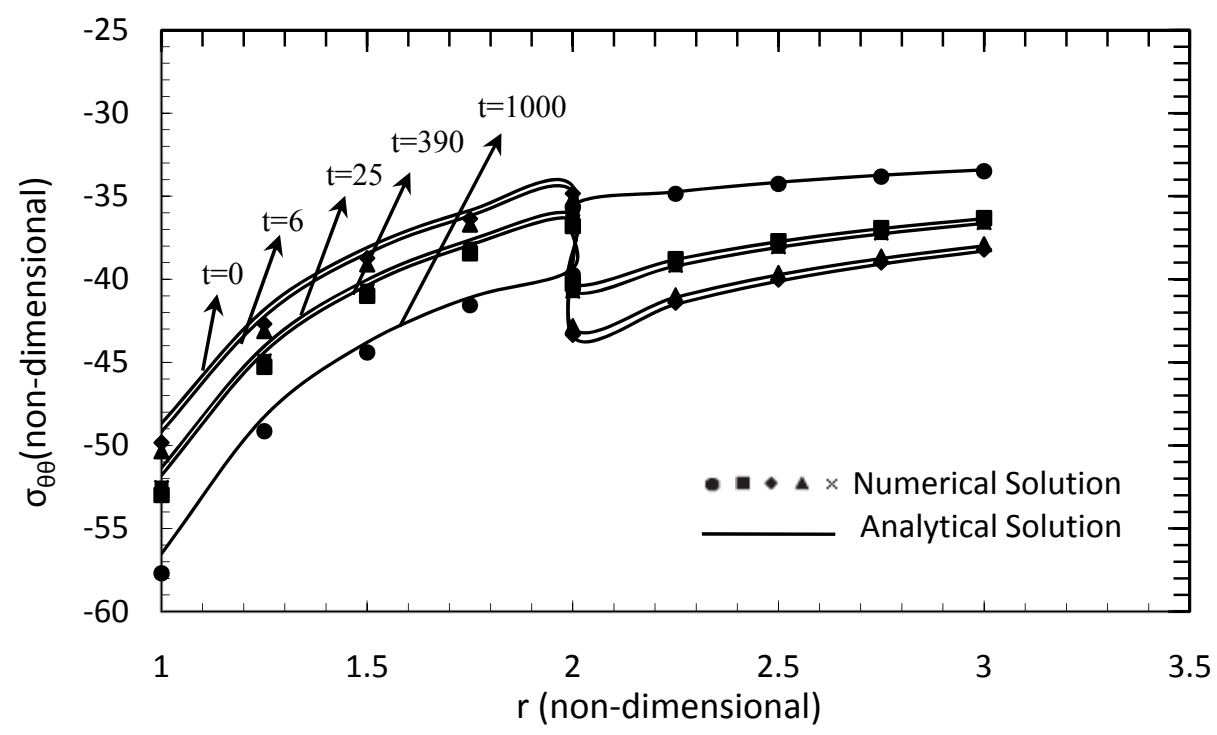

Fig. 3.8. Hoop stress in the composite cylinder as a function of time 


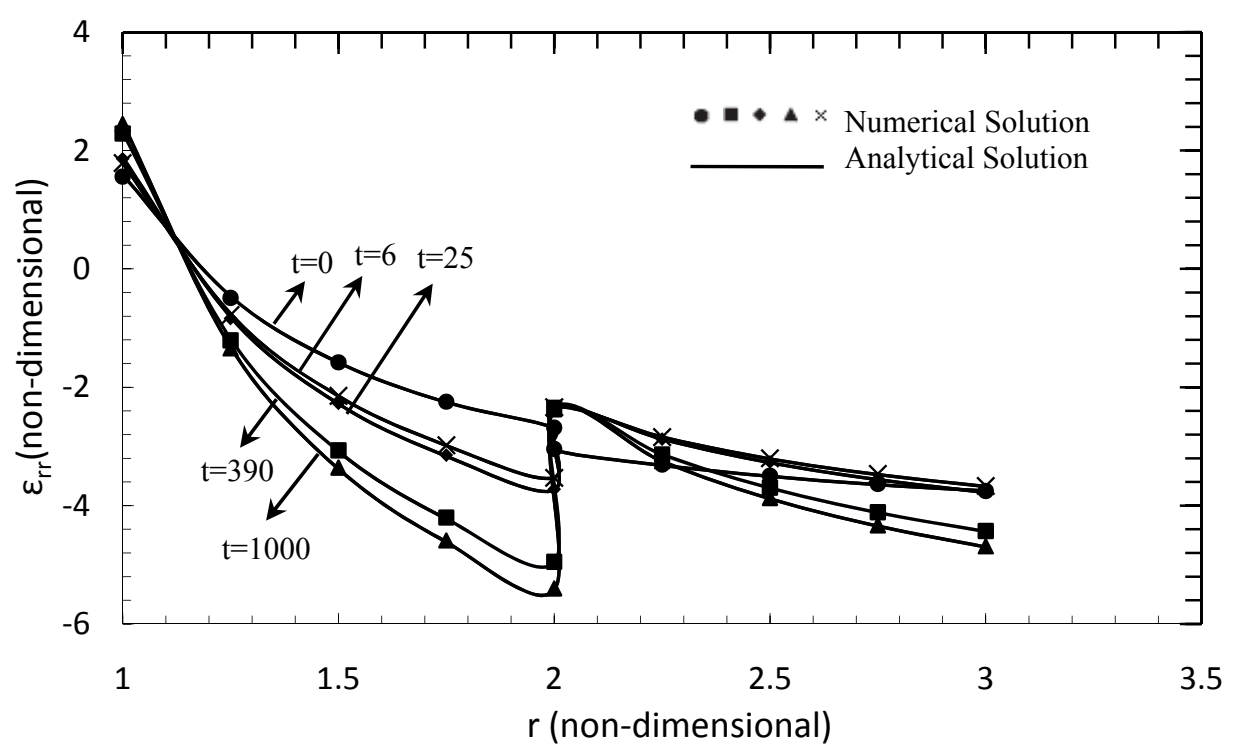

Fig. 3.9. Radial strain in the composite cylinder as a function of time

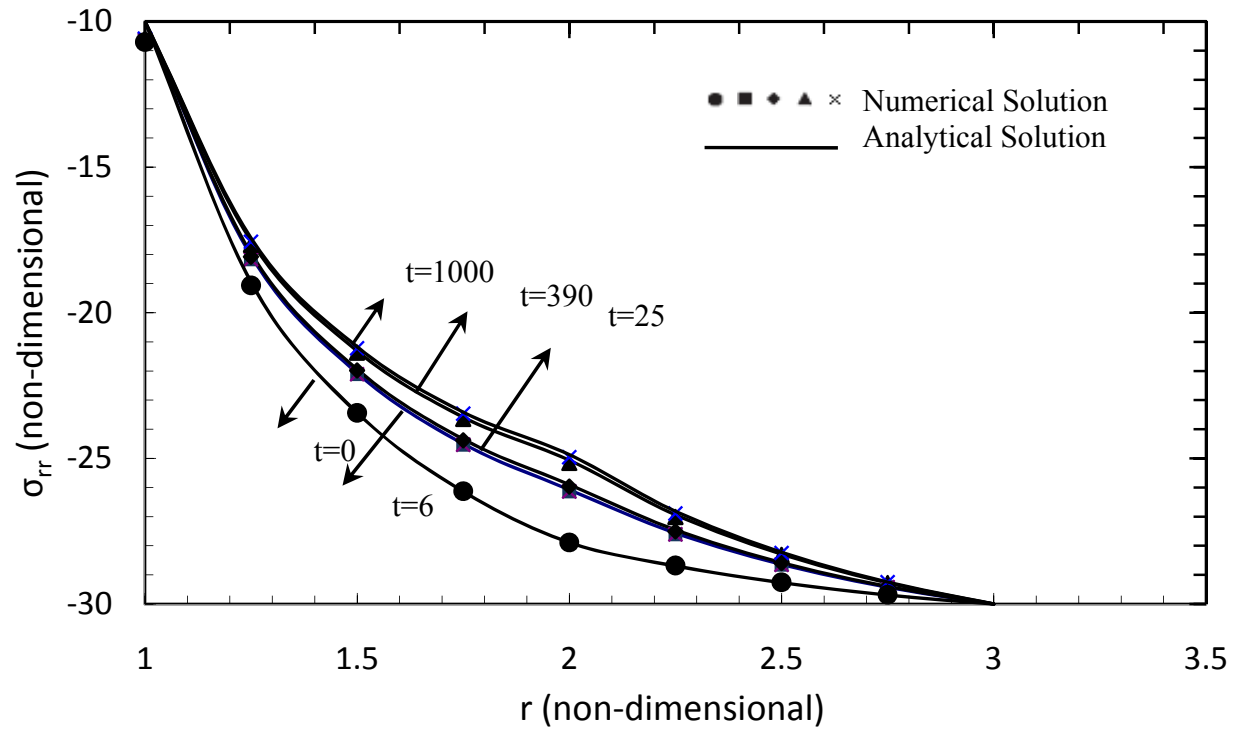

Fig. 3.10. Radial stress in the composite cylinder as a function of time 


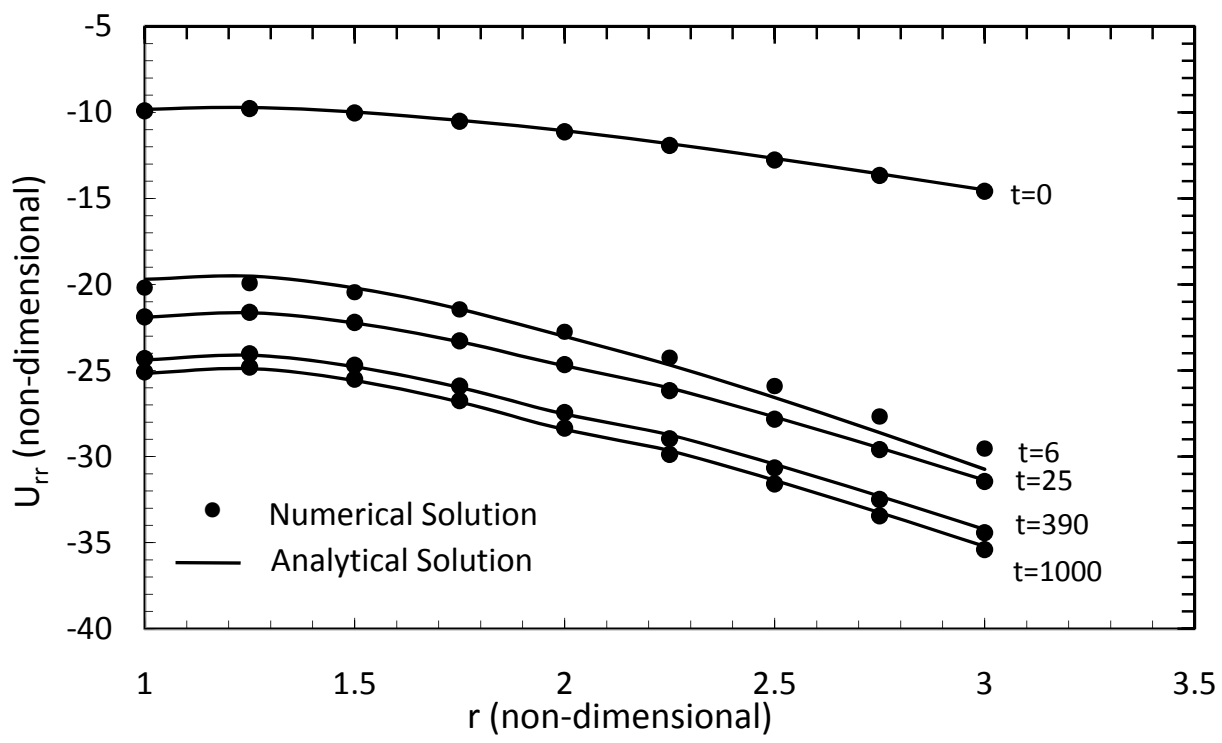

Fig. 3.11. Radial displacement for composite cylinder with temperature dependent properties as a function of time

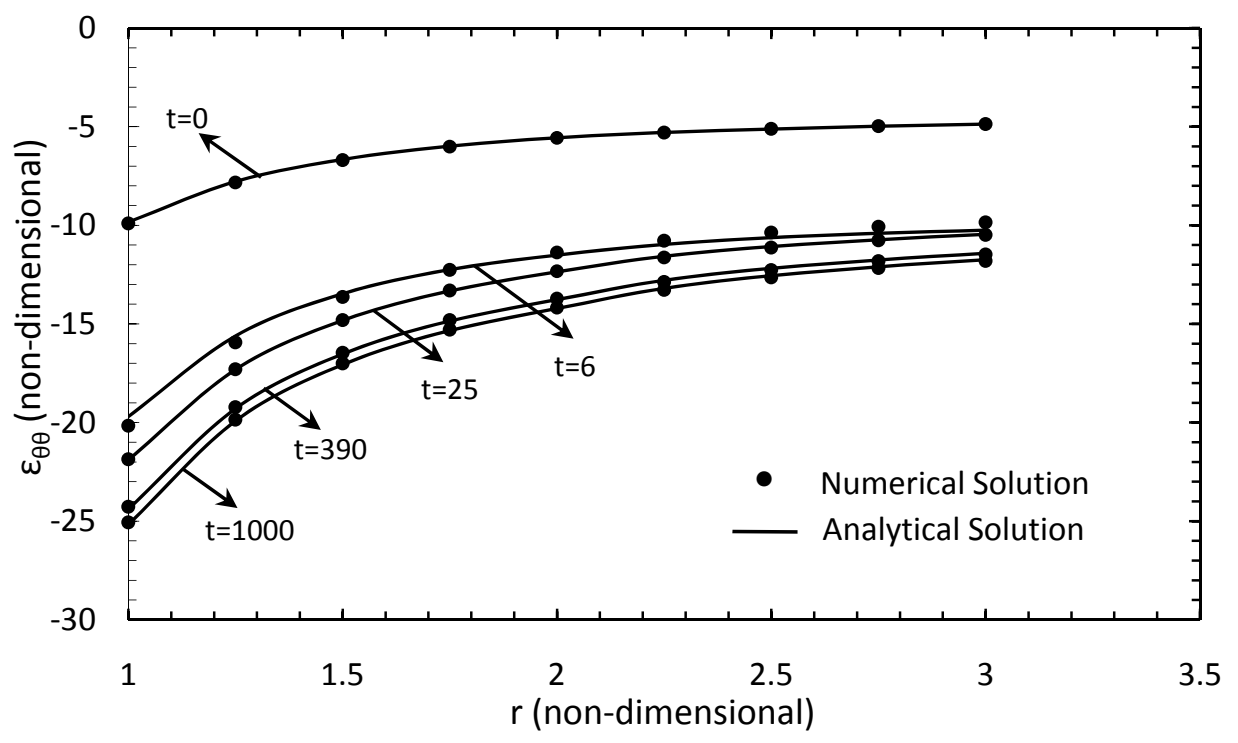

Fig. 3.12. Hoop strain for composite cylinder with temperature dependent properties as a function of time 


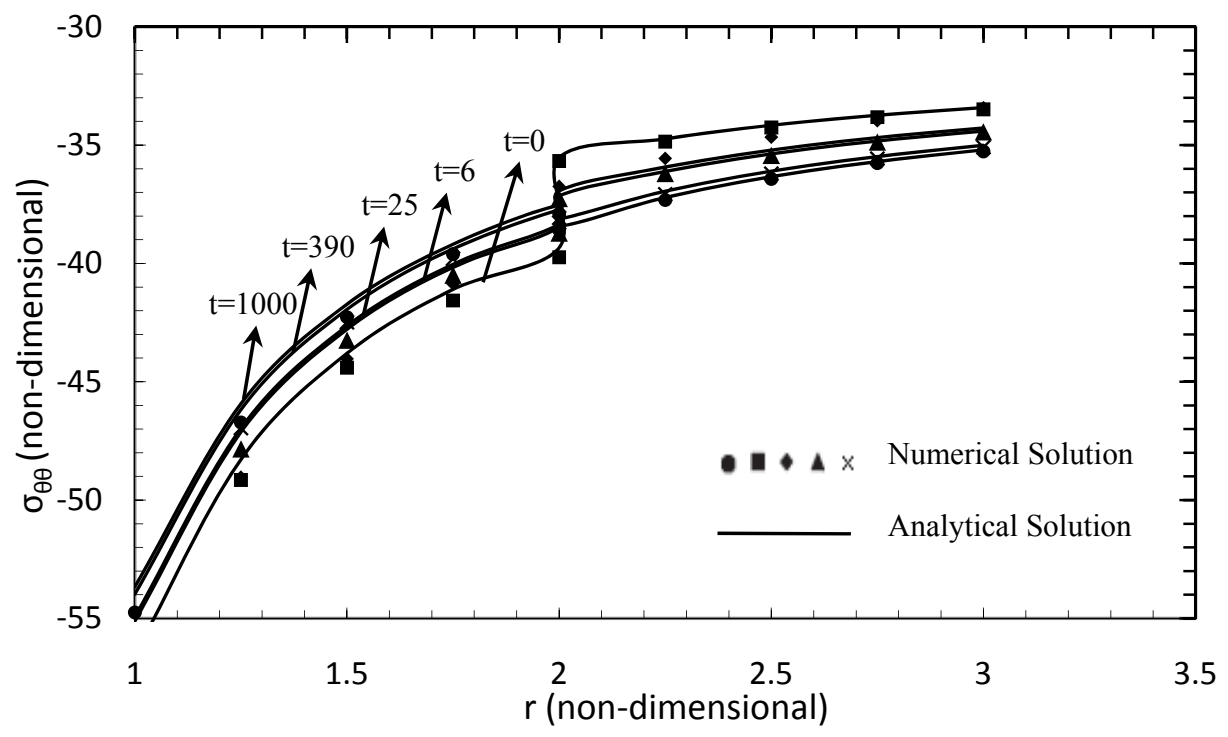

Fig. 3.13. Hoop stress for the composite cylinder with temperature dependent properties as a function of time

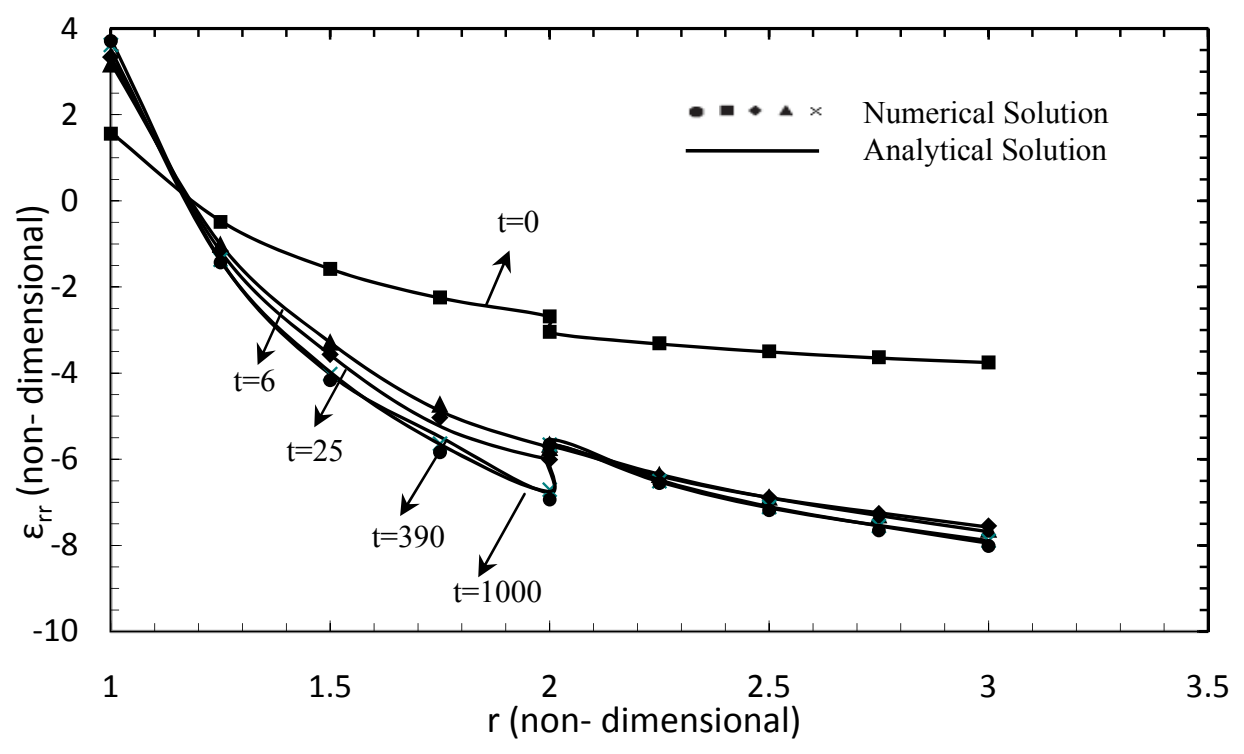

Fig. 3.14. Radial strain for the composite cylinder with temperature dependent properties as a function of time 


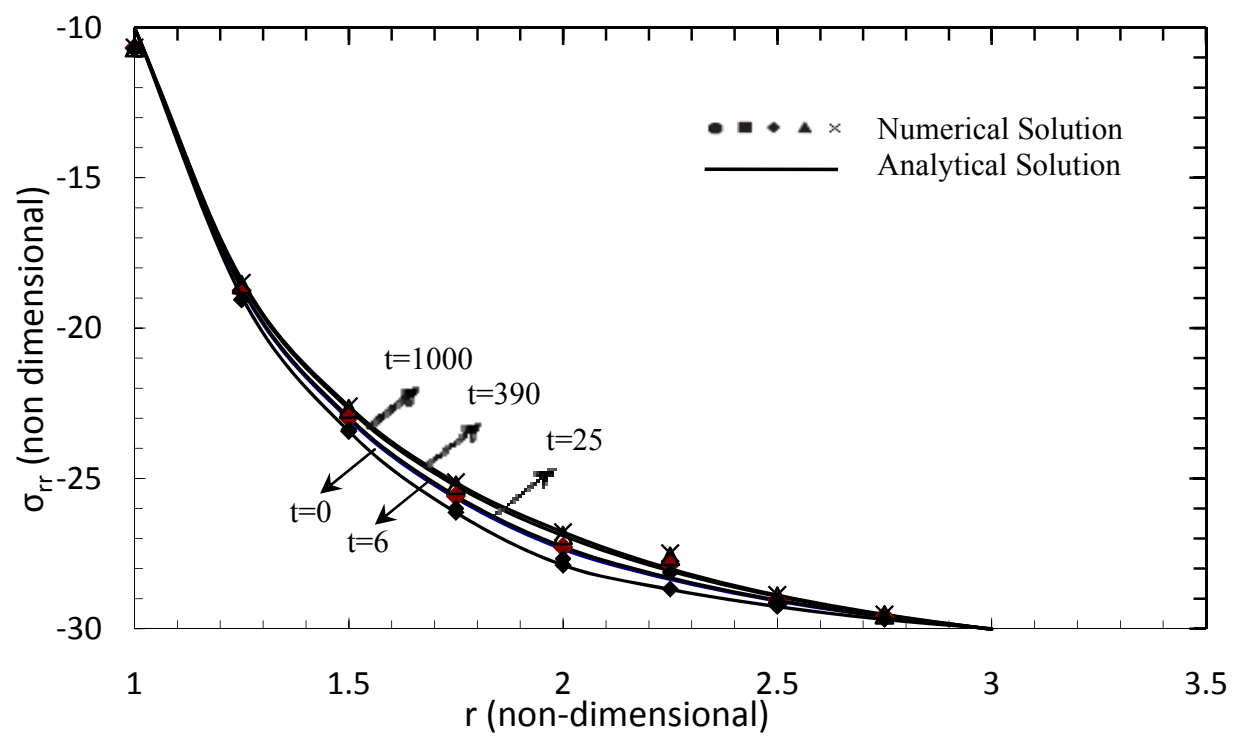

Fig. 3.15. Radial stress for the composite cylinder with temperature dependent properties as a function of time

The radial displacement as seen in the Fig. 3.6, at the interface at $r=2.0$ is -11.28 units at $\mathrm{t}=0$ which increases to -21.49 at $\mathrm{t}=1000$ in case 1 i.e. it approximately increases by $91 \%$ in the composite viscoelastic cylinder which shows the pronounced effect of viscoelasticity on the response. When the temperature dependent material properties are considered as in case 2 the displacement at the interface as shown in Fig. 3.11, at $r=2.0$ at time $=1000$ becomes -28.34 which is approximately an increase of $151 \%$. This shows that the temperature dependence of the material properties significantly affects the displacement field developed in the material.

The value of hoop strain induced at the interface goes on increasing with increasing time. The value increases by around $93 \%$ at $\mathrm{t}=1000$ when compared with the 
one at $\mathrm{t}=0$ in case 1 as shown in Fig. 3.7. Also as expected, higher hoop strain is induced when the temperature effect is incorporated in case 2 . The increment in the value at $\mathrm{r}=2.0$ is as high as $165 \%$ at $\mathrm{t}=1000$ as compared to the value at $\mathrm{t}=0$ as seen in the Fig.

\subsection{2 in case 2.}

The hoop stress induced in the cylinder has a discontinuity at the interface of $\mathrm{r}=2.0$. In the case of temperature independent properties in case 1, the magnitude of the jump at the interface goes on increasing with increasing time as seen in the Fig. 3.8. It is interesting to notice from the Fig. 3.13, that the magnitude of the jump at the interface actually decreases with increasing time in case 2 , i.e. it decreases from 4.0 at time $t=0$ to approximately 0.7 at time $\mathrm{t}=1000$. Thus if the maximum value of the jump that occurs during the initial time is lower than the critical value, then it is assured that with increasing time the chances of delamination of the two layers due to discontinuity of the hoop stress are very less in case 2 where material properties are temperature dependent. That is to say that the maximum value of jump that occurs during the initial time of transient heat transfer should be within the maximum permissible value to avoid delamination due to discontinuity in hoop stress.

The increase in the radial strain as shown in Fig. 3.14, at the interface at $r=2.0$ can be as high as $135 \%$ at $\mathrm{t}=1000$ when material properties are made temperature dependent in case 2 as against the case of temperature independent material properties in case 1 shown in Fig. 3.9. Also as expected there is a discontinuity in the radial strain at the interface, magnitude of which goes on increasing with time in both case 1 and 2 . It is also observed that the radial strain at $r=1.0$ is positive in virtue of the pressure being 
applied at that boundary. With the temperature dependent properties as in case 2 , the actual values of radial strains induced are higher than the case of temperature independent material properties in case 1, for example, the maximum strain induced is at $\mathrm{r}=3.0$ at $\mathrm{t}=1000$ is around 8.0 units as against 4.7 units in temperature independent case. But it is noticed that the actual magnitude of the jump in case 2 is less than the values in case 1. The actual magnitude depends on the form of temperature dependence that is assumed, were we to choose more severe dependence than the linear form, different values of strains would have been induced. This type of dependence is chosen so as to illustrate that the effect of temperature can be pronounced.

The stress field developed depends on the material properties and thus it changes with time. The radial stress at the interface decreases from initial value of 27.88 at $t=0$ to 26.7 at $\mathrm{t}=1000$ in the case 1 as seen in Fig. 3.10. As expected with temperature dependent properties in case 2, the amount of decrease is more i.e. it changes from 27.8 to 25 units at $t=1000$ as shown in Fig. 3.15. Also, there is the radial stress continuity at the interface which is in accordance to the traction continuity condition imposed at the interface while developing the analytical solution. 


\section{CHAPTER IV}

\section{THE EFFECT OF MATERIAL PARAMETERS IN THE VISCOELASTIC CONSTITUTIVE MODELS ON THE OVERALL RESPONSE OF THE COMPOSITE CYLINDER}

This chapter presents parametric studies carried out to understand the effect of different material parameters in the viscoelastic constitutive models on the overall response of the composite cylinder. In the first three sections, the material properties of the layer 1 are kept constant as previously studied in chapter III and the material properties of the layer 2 are varied. It is done to understand the effect of each individual material parameter namely instantaneous (elastic) part, Prony coefficients in the transient part of the creep compliance and the characteristic of creep time, on the stress, strain and displacement fields. In the last section, the effect of the temperature dependent function i.e. linear and quadratic polynomial forms of temperature dependence of the material properties on the overall response of the composite cylinder is analyzed. In all the studies that have been performed, the mechanical response in terms of stress, strain and displacement field is studied. This is done as the composite cylinder under consideration is made up of two different materials and hence at the interface due to mismatch in material properties, there arises a discontinuity in the hoop stress and radial strain. If this value of the jump exceeds the critical value then it may lead to delamination of the two layers which is generally not desired. Also, in certain design applications, it is not desirable if the maximum deformation of the body exceeds certain values depending on the type of application. Therefore, the radial displacement is also evaluated. 


\subsection{EFFECT OF VARYING PRONY COEFFIECIENTS IN THE CREEP COMPLIANCE ON THE OVERALL RESPONSE OF THE COMPOSITE CYLINDER}

This study is aimed at assessing the effect of varying the material property by altering Prony coefficients in the transient creep compliance, on the overall response of viscoelastic composite cylinder in terms of stress, strain and displacement fields. Three different cases are considered, each having different value of transient creep parameters of layer 2. Case 1 has the material properties of layer 2 as mentioned in chapter III. In the case 2, the Prony coefficients for layer 2 have the magnitude which is half of the value of the corresponding Prony coefficients in the case 1 and in the case 3 the magnitude of Prony coefficients is three times higher than the corresponding Prony terms in case 1. The Table 4.1 gives the elastic and transient creep compliance parameters of the two layers in all three cases. 
Table 4.1. Material parameters for three different cases by changing the prony coefficients

\begin{tabular}{|c|c|c|c|c|c|c|}
\hline \multirow{6}{*}{ Case 1} & \multicolumn{3}{|c|}{ Layer 1} & \multicolumn{3}{|c|}{ Layer 2} \\
\hline & $\mathrm{n}$ & $\lambda_{n}$ & $D_{n}$ & $\mathrm{n}$ & $\lambda_{\mathrm{n}}$ & $\mathrm{D}_{\mathrm{n}}$ \\
\hline & 1 & 1 & 0.07 & 1 & 1 & 0.01 \\
\hline & 2 & 0.01 & 0.09 & 2 & 0.01 & 0.05 \\
\hline & 3 & 0.001 & 0.12 & 3 & 0.001 & 0.07 \\
\hline & \multicolumn{3}{|c|}{$\mathrm{D}_{0}=0.195$} & \multicolumn{3}{|c|}{$\mathrm{D}_{0}=0.25$} \\
\hline \multirow{6}{*}{ Case 2} & \multicolumn{3}{|c|}{ Layer 1} & \multicolumn{3}{|c|}{ Layer 2} \\
\hline & $\mathrm{n}$ & $\lambda_{\mathrm{n}}$ & $\mathrm{D}_{\mathrm{n}}$ & $\mathrm{n}$ & $\lambda_{\mathrm{n}}$ & $\mathrm{D}_{\mathrm{n}}$ \\
\hline & 1 & 1 & 0.07 & 1 & 1 & 0.005 \\
\hline & 2 & 0.01 & 0.09 & 2 & 0.01 & 0.025 \\
\hline & 3 & 0.001 & 0.12 & 3 & 0.001 & 0.035 \\
\hline & \multicolumn{3}{|c|}{$\mathrm{D}_{0}=0.195$} & \multicolumn{3}{|c|}{$\mathrm{D}_{0}=0.25$} \\
\hline \multirow{6}{*}{ Case 3} & \multicolumn{3}{|c|}{ Layer 1} & \multicolumn{3}{|c|}{ Layer 2} \\
\hline & $\mathrm{n}$ & $\lambda_{\mathrm{n}}$ & $\mathrm{D}_{\mathrm{n}}$ & $\mathrm{n}$ & $\lambda_{\mathrm{n}}$ & $\mathrm{D}_{\mathrm{n}}$ \\
\hline & 1 & 1 & 0.07 & 1 & 1 & 0.03 \\
\hline & 2 & 0.01 & 0.09 & 2 & 0.01 & 0.15 \\
\hline & 3 & 0.001 & 0.12 & 3 & 0.001 & 0.21 \\
\hline & \multicolumn{3}{|c|}{$\mathrm{D}_{0}=0.195$} & \multicolumn{3}{|c|}{$\mathrm{D}_{0}=0.25$} \\
\hline
\end{tabular}

In Table $4.1, n$ is the number of Prony terms, $D_{n}$ is the $n^{\text {th }}$ Prony coefficient, $\lambda_{n}$ is the reciprocal of the characteristic of the $\mathrm{n}^{\text {th }}$ retardation time and $\mathrm{D}_{0}$ is the elastic part of creep compliance.

Fig. 4.1 shows the plot of creep compliance of layer 1 and layer 2 for all the three cases that are considered above, where D1 denotes the creep compliance of layer 1 and 
D2 denotes the creep compliance of layer 2. It is seen from the Fig. 4.1 that in case 2, layer 2 (external layer) shows nearly elastic response.

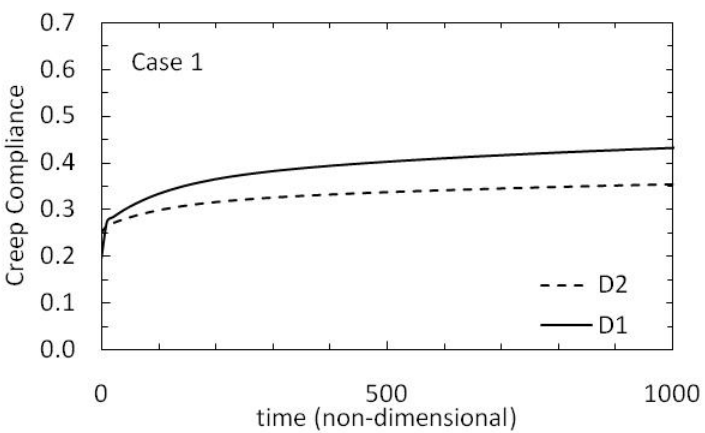

(a)

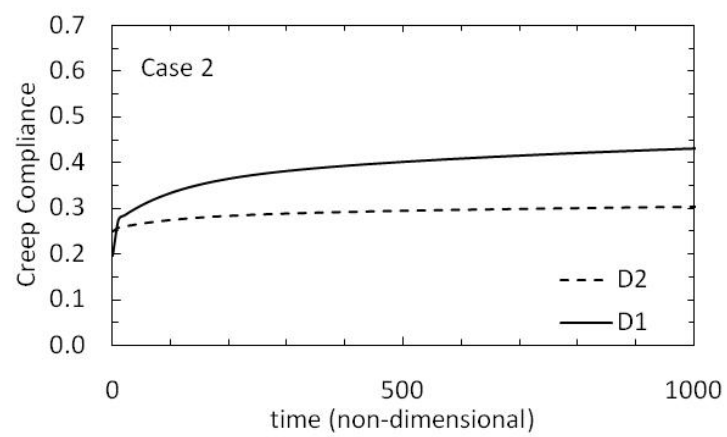

(b)

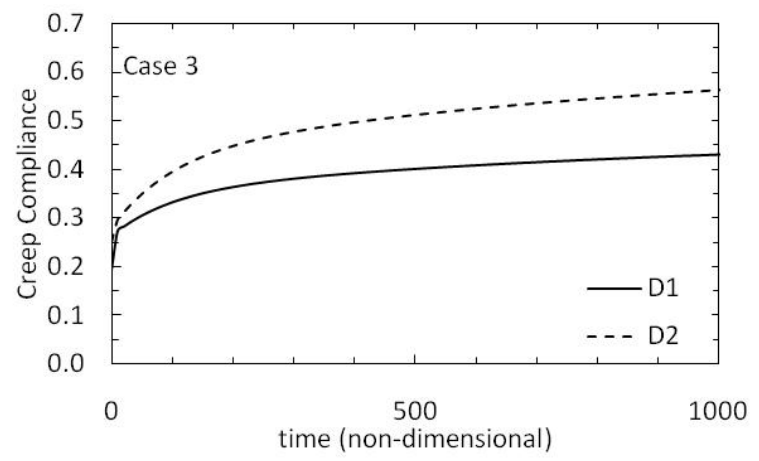

(c)

Fig. 4.1. Creep compliance of the two layers with time for all three cases

The material properties of both layers as mentioned in Table 4.1 have identical temperature dependent function as discussed in chapter III. This is done to isolate the time-dependent effect from the combined time and temperature variations. The material properties are assumed to have linear dependence on temperature given by Eqs. (3.6) and (3.7). Since the traction and displacement continuity is always enforced at the interface, 
as mentioned in Eq. (2.17), the radial stress and hoop strain are continuous across the interface and not much effect is seen on those responses by varying the material properties. Thus, in the following discussion the material response is described in terms of hoop stress, radial strain, and radial displacement.

Fig. 4.2 shows hoop stresses in the body at different times for the three cases. As discussed in chapter III, a discontinuity in the hoop stress is observed at the interface at $\mathrm{r}=2.0$. As shown in the Fig. $4.2 \mathrm{a}$, the hoop stresses developed at the initial times, i.e. at $\mathrm{t}=2$ in the three cases is almost the same which is as expected, as during the initial times, the contribution of the transient part of creep compliance on the overall material property is not significant as seen from Fig. 4.1. As the time advances, the jump at the interface at $r=2.0$ goes on increasing in case 3 whereas in the case 1 and case 2 , the jump decreases upto certain time and then again increases. The magnitude of this jump at the interface is maximum in case 3 as compared to case 1 and case 2 at all the times. As seen from the Fig. 4.2.b, at time $t=62$, the jump at the interface is much less for case 1 and almost negligible in case 2 as compared to case 3 . At time $t=251$, there is negligible jump of 0.04 units in case 1 as compared to 4.0 units in case 3 . At $t=1000$ units, the magnitude of jump at the interface in case 3 is the maximum of 4.5 units as against 2.2 units in case 2 and 0.66 in case 1 which is the least. This type of pattern can be expected as the difference in the material properties of two layers at all the times is the most in the case 3 and the least in the case 1 . It shows that change in the transient part of creep compliance can alter the magnitude of the jump at the interface significantly at longer times. The decrease in the magnitude of the jump in hoop stress at the interface 
at $\mathrm{r}=2.0$ in case 1 and case 2 upto certain time as seen from the Fig. 4.2.b and 4.2.c, can be used to avoid debonding due to high stress discontinuities. Also understanding the stress discontinuity with respect to time in all three cases with different material parameters can be useful in design aspects to ensure sufficient interface bonding upto desired time for the type of materials selected.
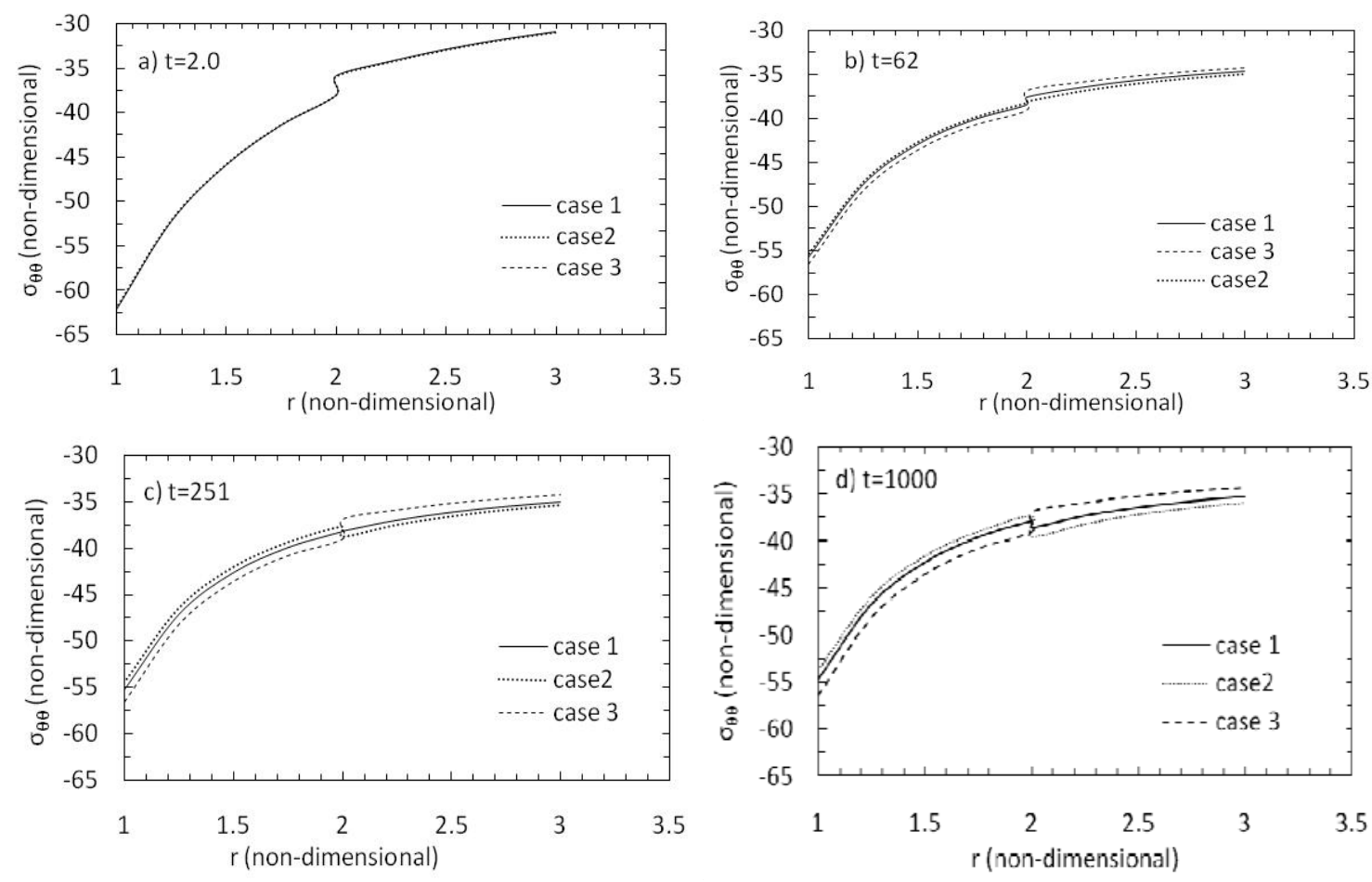

Fig. 4.2. Hoop stress for the composite cylinder at different times for different Prony coefficients in layer 2

The radial strain developed across the body at different times in all the cases is shown in Fig. 4.3. During the initial times i.e. at $\mathrm{t}=2$, the radial strains developed are 
almost the same in all three cases as seen in Fig. 4.3.a. As the time progresses, the magnitude of the jump in the radial strain at the interface at $r=2.0$ goes on increasing in all three cases. The magnitude of the jump at $r=2.0$ in case 2 is 1.87 units which is the maximum compared to the 1.28 units in case 1 and 1.2 units in case 3 at $t=1000$ units. In other words, the jump in case 2 is $46 \%$ higher compared to case 1 and $56 \%$ higher compared to case 3 as seen in the Fig. 4.3.d. The actual magnitude of the radial strain in the case 3 is higher as compared to case 1 and case 2 at all the times. At time $t=1000$,
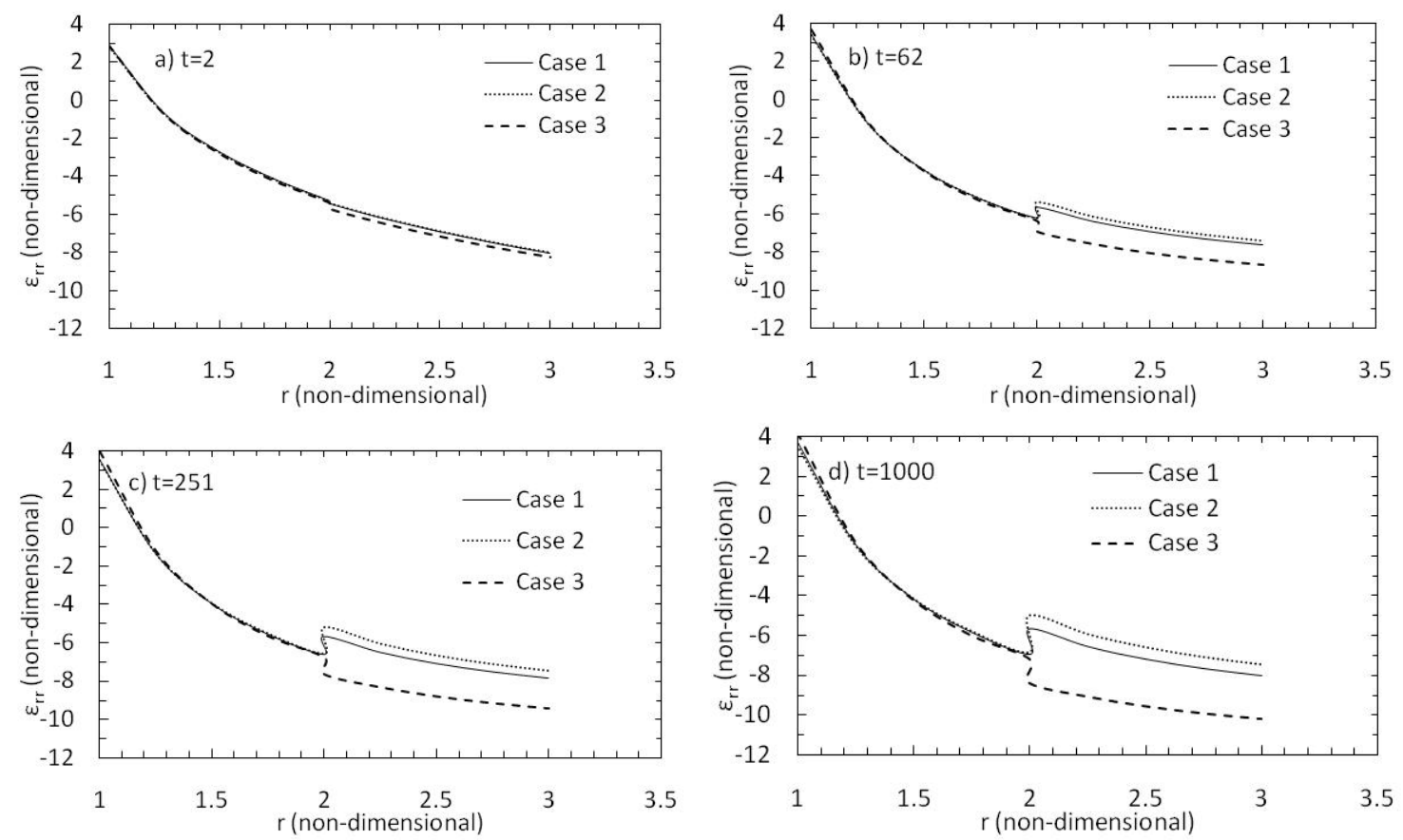

Fig. 4.3. Radial strain for the composite cylinder at different times for different prony coefficients in layer 2 
magnitude of radial strain at $r=3.0$ in case 3 is approximately $14 \%$ higher compared to case 1 and $22 \%$ higher than case 2 . It shows that the effect of different Prony coefficients in the transient creep compliance on the overall radial strain can be pronounced at longer times.

Fig. 4.4 shows the radial displacement of the body for different times in all three cases. At the initial time, i.e. at $\mathrm{t}=2$, no significant difference is observed in the radial displacement in the case 1 and case 2, but slightly higher displacement is observed in the case 3 as seen in Fig. 4.4.a. At $\mathrm{t}=2$, the value of radial displacement at $\mathrm{r}=3.0$ in case 3
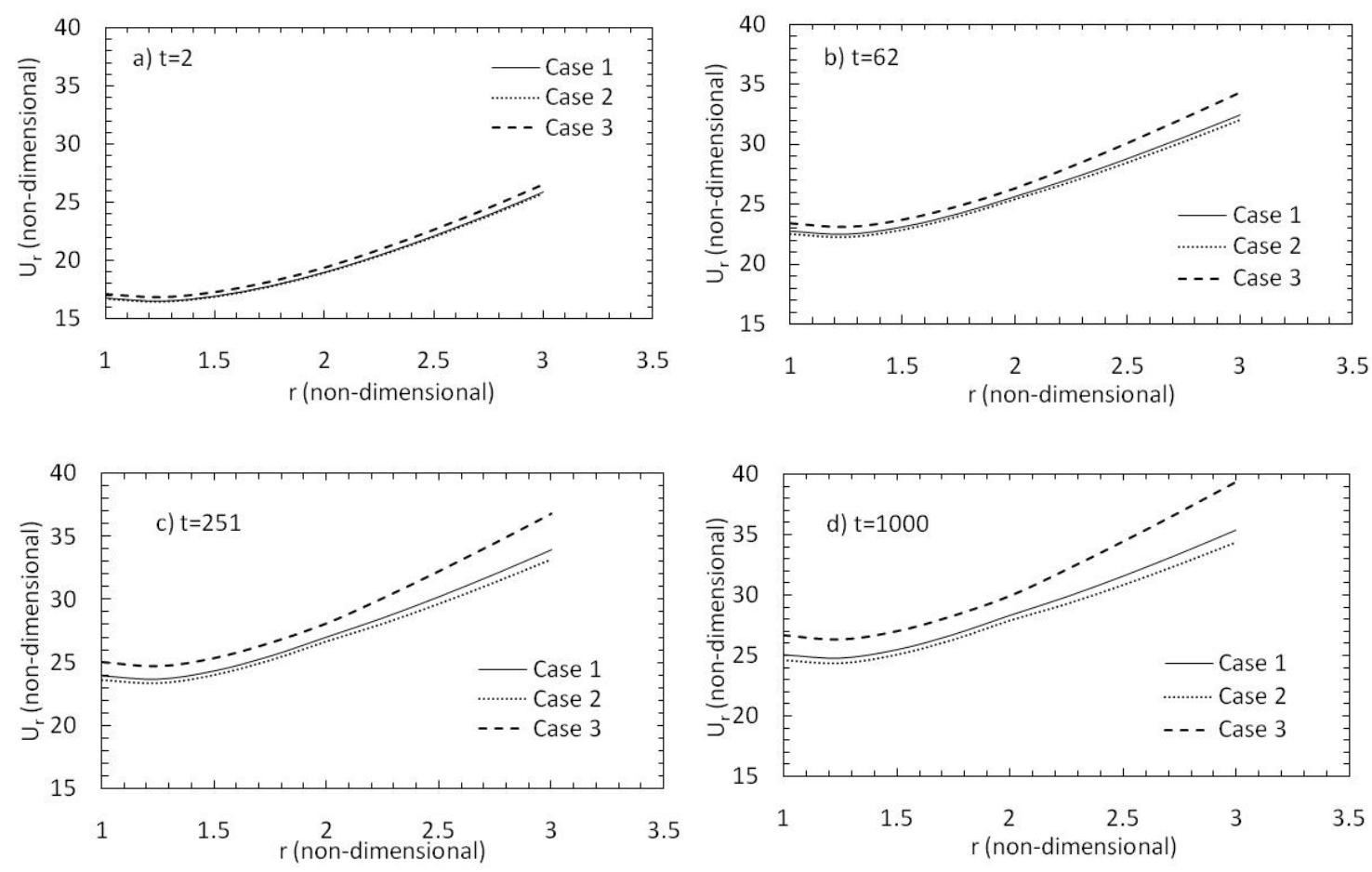

Fig. 4.4. Radial displacement for the composite cylinder at different times for different prony coefficients in layer 2 
is $2.5 \%$ higher as compared to the one in case 1 and case 2 . The maximum of radial displacement is observed for the case 3 at all times. The increase in the radial displacement in case 3 is as high as $11 \%$ when compared to case 1 and approximately $15 \%$ when compared to case 2 at time $\mathrm{t}=1000$ at $\mathrm{r}=3.0$. This shows that the effect of changing the Prony coefficients in transient creep compliance of the material of layer 2 can be significant on the radial displacement at longer times.

\subsection{EFFECT OF DIFFERENT CHARACTERISTIC OF CREEP TIME IN THE CREEP COMPLIANCE ON THE OVERALL RESPONSE}

This study is carried out to understand the effect of the characteristic of creep time $\left(\lambda_{n}\right)$ in the creep compliance on the overall material response. Similar to the study done in section 4.1, material parameters of layer 1 are kept constant while the material parameters of layer 2 are varied by changing the characteristic creep time. Three different cases are considered. Case 1 has the material properties of layer 2 as mentioned in chapter III. In the case 2 , the values of $\lambda_{n}$ are four times the corresponding values in case 1 , and in case 3 the value of $\lambda_{n}$ is one fourth of that of the corresponding values in case 1 . Table 4.2 shows the elastic and transient creep parameters of the materials in both the layers for three cases where $n$ is the number of Prony terms, $D_{n}$ is the $\mathrm{n}^{\text {th }}$ Prony coefficient, $\lambda_{\mathrm{n}}$ is the reciprocal of the characteristic of the $\mathrm{n}^{\text {th }}$ retardation time and $\mathrm{D}_{0}$ is the elastic compliance. The material properties of both layers are temperature dependent, as discussed in chapter III. 
Table 4.2. Elastic and transient material properties of two layers for all the cases

\begin{tabular}{|c|c|c|c|c|c|c|}
\hline \multirow{6}{*}{ Case 1} & \multicolumn{3}{|c|}{ Layer 1} & \multicolumn{3}{|c|}{ Layer 2} \\
\hline & $\mathrm{n}$ & $\lambda_{\mathrm{n}}$ & $\mathrm{D}_{\mathrm{n}}$ & $\mathrm{n}$ & $\lambda_{\mathrm{n}}$ & $\mathrm{D}_{\mathrm{n}}$ \\
\hline & 1 & 1 & 0.07 & 1 & 1 & 0.01 \\
\hline & 2 & 0.01 & 0.09 & 2 & 0.01 & 0.05 \\
\hline & 3 & 0.001 & 0.12 & 3 & 0.001 & 0.07 \\
\hline & \multicolumn{3}{|c|}{$\mathrm{D}_{0}=0.195$} & \multicolumn{3}{|c|}{$\mathrm{D}_{0}=0.25$} \\
\hline \multirow{6}{*}{ Case 2} & \multicolumn{3}{|c|}{ Layer 1} & \multicolumn{3}{|c|}{ Layer 2} \\
\hline & $\mathrm{n}$ & $\lambda_{\mathrm{n}}$ & $\mathrm{D}_{\mathrm{n}}$ & $\mathrm{n}$ & $\lambda_{\mathrm{n}}$ & $\mathrm{D}_{\mathrm{n}}$ \\
\hline & 1 & 1 & 0.07 & 1 & 4 & 0.01 \\
\hline & 2 & 0.01 & 0.09 & 2 & 0.04 & 0.05 \\
\hline & 3 & 0.001 & 0.12 & 3 & 0.004 & 0.07 \\
\hline & \multicolumn{3}{|c|}{$\mathrm{D}_{0}=0.195$} & \multicolumn{3}{|c|}{$\mathrm{D}_{0}=0.25$} \\
\hline \multirow{6}{*}{ Case 3} & \multicolumn{3}{|c|}{ Layer 1} & \multicolumn{3}{|c|}{ Layer 2} \\
\hline & $\mathrm{n}$ & $\lambda_{\mathrm{n}}$ & $\mathrm{D}_{\mathrm{n}}$ & $\mathrm{n}$ & $\lambda_{\mathrm{n}}$ & $\mathrm{D}_{\mathrm{n}}$ \\
\hline & 1 & 1 & 0.07 & 1 & 0.25 & 0.01 \\
\hline & 2 & 0.01 & 0.09 & 2 & 0.025 & 0.05 \\
\hline & 3 & 0.001 & 0.12 & 3 & 0.0025 & 0.07 \\
\hline & \multicolumn{3}{|c|}{$\mathrm{D}_{0}=0.195$} & \multicolumn{3}{|c|}{$\mathrm{D}_{0}=0.25$} \\
\hline
\end{tabular}

The Fig. 4.5 shows the plot of creep compliance of layer 1 and layer 2 for all the three cases. D1 denotes the creep compliance of layer 1 and D2 denotes the creep compliance of layer 2 . 


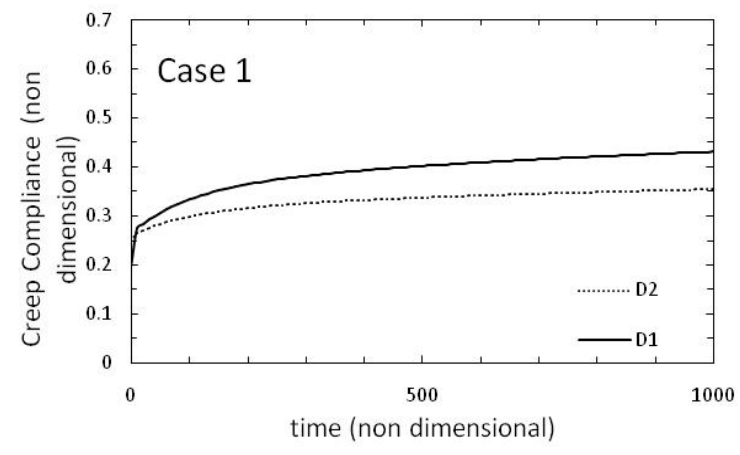

(a)

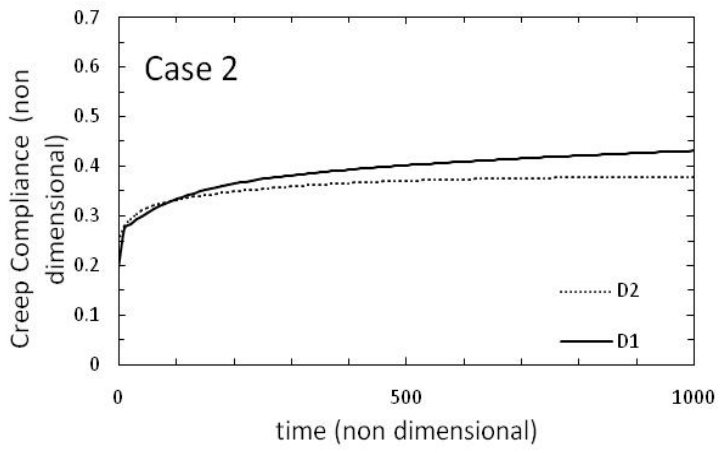

(b)

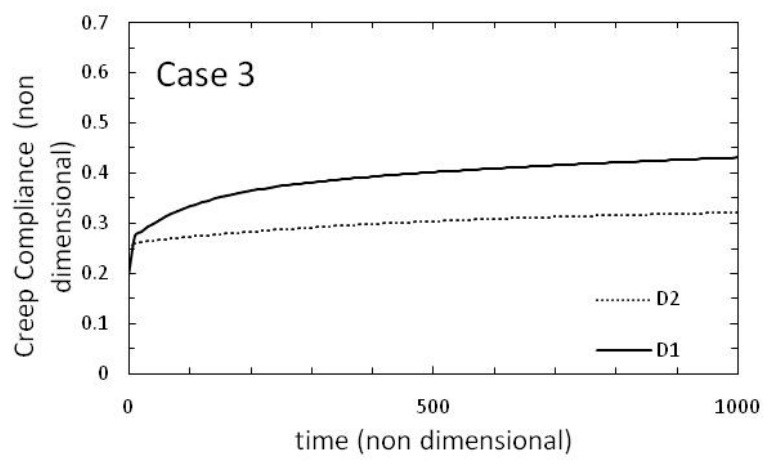

(c)

Fig. 4.5. Creep compliance of both the layers for all three cases

Fig. 4.6 shows the hoop stress induced in the composite cylinder at different times in the three cases. At the initial times, i.e. at $\mathrm{t}=2$ the jump in the hoop stress in all three cases is almost the same as seen from Fig. 4.6.a. At time $\mathrm{t}=62$, the jump at the interface at $\mathrm{r}=2.0$ in the case 3 is almost negligible. As the time increases the jump at the interface at $\mathrm{r}=2.0$ in case 3 goes on increasing. At $\mathrm{t}=1000$, the jump in hoop stress in case 3 is found to be maximum. The jump at the interface in the case 2 is almost $71 \%$ 
less and in case 1 is $56 \%$ less as compared to case 3 . The value of the jump in all three cases at all the times upto 1000 is still less as compared to the values of jump obtained by changing the Prony coefficient as seen from the figure 4.2. Thus the effect of change in the characteristic of creep time as seen from this study is not so significant as compared to the effect of change in Prony coefficient on the hoop stress as discussed in section 4.1.
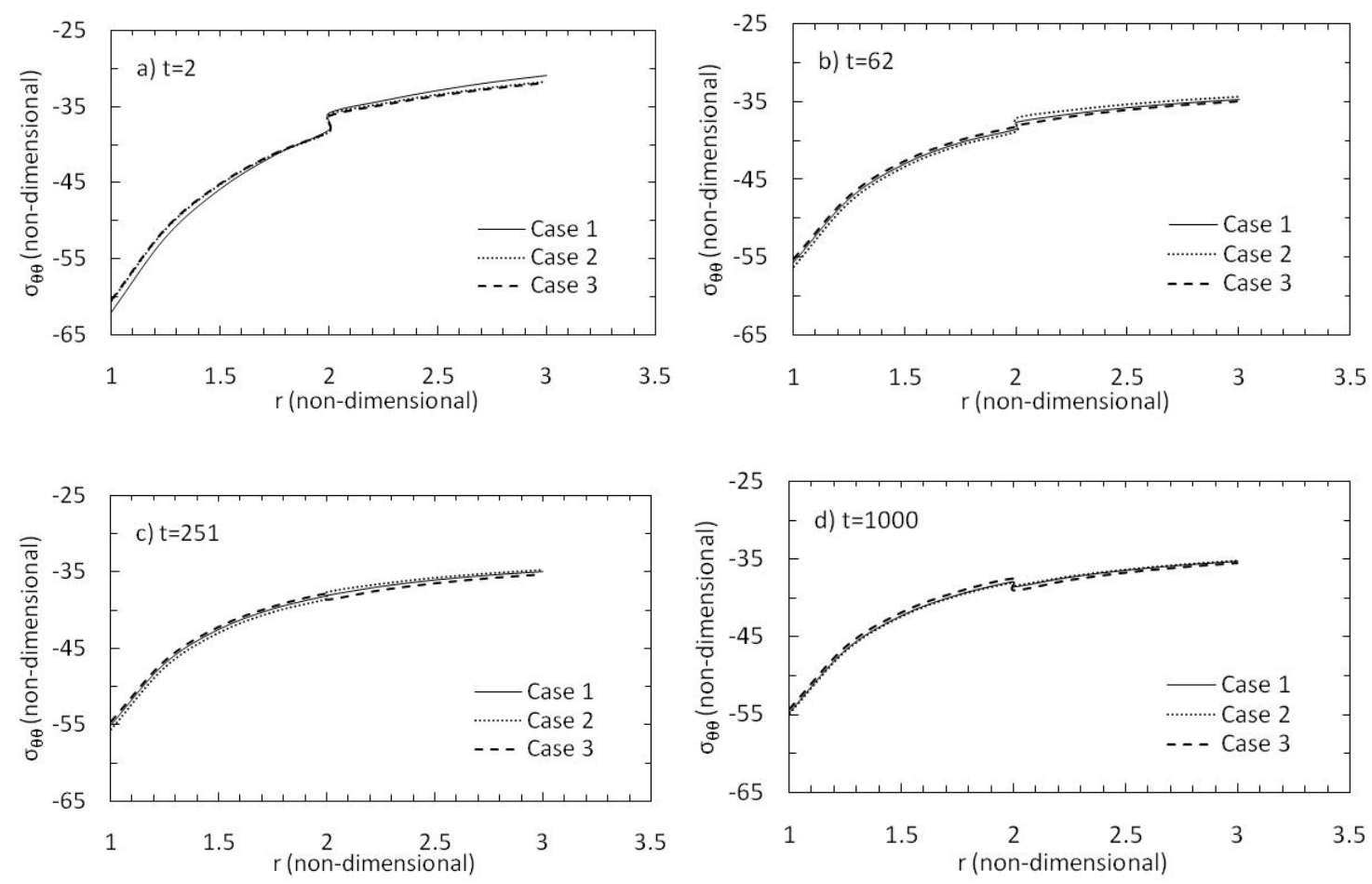

Fig. 4.6. Hoop stress for the composite cylinder at different times for different creep time in layer 2 
The radial strain developed in all three cases at different times is shown in Fig. 4.7. At initial times at around $t=2$, the values of radial strain are almost the same for the three cases as shown in Fig. 4.7.a. With increase in time, the jump in the radial strain at the interface at $r=2.0$ increases in all three cases. At $t=62$, the magnitude of the jump at the interface in the case 2 is almost $73 \%$ less as compared to the one in case 3 and in case 1 is $33 \%$ less as compared to the one in case 3 . The magnitude of the jump in the case 3 at time $\mathrm{t}=1000$ is $33 \%$ higher than the case 2 and almost $34 \%$ higher as compared to case 1 as seen in Fig. 4.7.d.
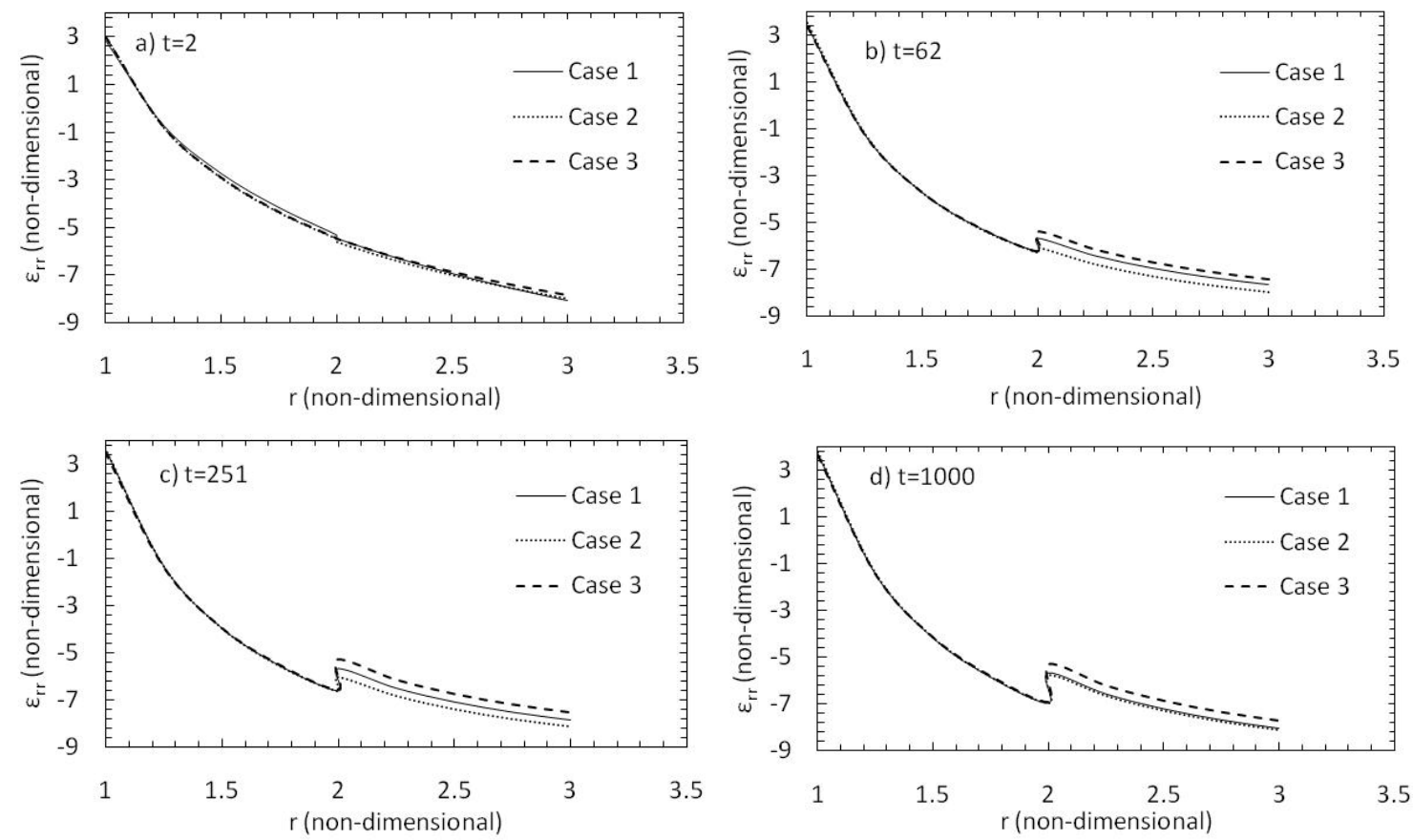

Fig. 4.7. Radial strain for the composite cylinder at different times for different creep time 
The effect of varying the characteristic of creep time on the radial displacement at different times is as shown in the Fig. 4.8. At initial time $\mathrm{t}=2$, the radial displacement at $\mathrm{r}=3.0$ in case 2 and case 3 is almost the same which is $5 \%$ higher as compared to case 1. At time $\mathrm{t}=62$, the maximum radial displacement is seen in case 2 as compared to case 1 and case 3. At longer time of $t=1000$, the value of radial displacement at $r=3.0$ is almost the same in case 2 and case 1 which is $3 \%$ higher as compared to case 3 . The effect of varying the characteristics creep time on the radial displacement is this study is not so significant as compared to the effect of varying Prony coefficients as seen in section 4.1.
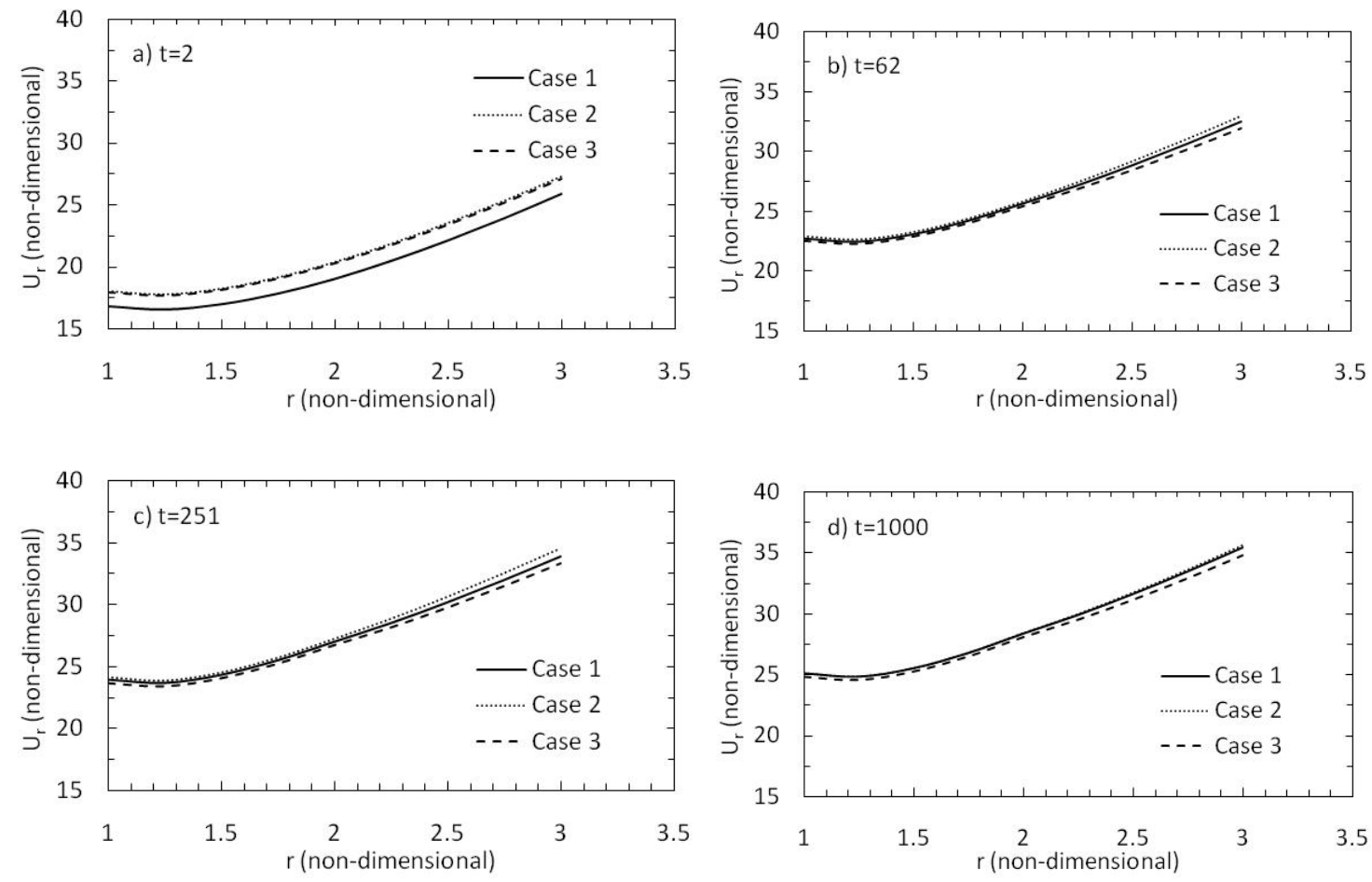

Fig. 4.8. Radial displacement for the composite cylinder at different times for different creep time 


\subsection{EFFECT OF DIFFERENT ELASTIC CONSTANTS ON THE OVERALL RESPONSE OF THE COMPOSITE CYLINDER}

The effect of the elastic material constants on the overall response of the composite cylinder is studied. To this end, the elastic part of compliance of the layer 2 is varied and the material parameters of the layer 1 are kept constant. Four different cases are considered. The Table 4.3 shows the transient compliances of the layer 1 and layer 2 , which remain the same for all four cases, where $n$ is the number of Prony terms, $D_{n}$ is the $n^{\text {th }}$ Prony coefficient and $\lambda_{n}$ is the reciprocal of the characteristic of the $n^{\text {th }}$ retardation time. Table 4.4 shows the instantaneous elastic modulus of the layer 1 and layer 2 in all four cases:

Table 4.3. Transient creep properties for the material of layer 1 and layer 2 in all four cases

\begin{tabular}{|c|c|c|c|c|c|}
\hline \multicolumn{3}{|c|}{ Layer 1 } & \multicolumn{3}{c|}{ Layer 2 } \\
\hline \multirow{2}{*}{$\mathrm{n}$} & $\lambda_{\mathrm{n}}$ & $\mathrm{D}_{\mathrm{n}}$ & $\mathrm{n}$ & $\lambda_{\mathrm{n}}$ & $\mathrm{D}_{\mathrm{n}}$ \\
\cline { 2 - 6 } 1 & 1 & 0.07 & 1 & 1 & 0.01 \\
2 & 0.01 & 0.09 & 2 & 0.01 & 0.05 \\
3 & 0.001 & 0.12 & 3 & 0.001 & 0.07 \\
\hline
\end{tabular}


Table 4.4. Elastic properties of the material of layer 1 and layer 2

\begin{tabular}{|c|c|c|}
\hline & & \\
& Layer 1 & Layer 2 \\
\hline Case 1 & $\mathrm{E}_{0}=5.128$ & \\
\hline Case 2 & & $\mathrm{E}_{0}=4.0$ \\
\hline Case 3 & $\mathrm{E}_{0}=5.128$ & \\
\hline Case 4 & & $\mathrm{E}_{0}=2.0$ \\
\hline & $\mathrm{E}_{0}=5.128$ & $\mathrm{E}_{0}=3.5$ \\
\hline
\end{tabular}

Case 1 has the material properties as discussed in chapter III. The case 3 is considered to analyze the effect of minor change in elastic properties of the two layers as compared to case 1 on the overall response. Case 2 and case 4 are considered to analyze the effect of significant variations in the elastic properties as compared to case 1 on the overall response. Fig. 4.9 shows the comparisons of creep compliance of layer 1 denoted by D1 and creep compliance of layer 2 denoted by D2 for all the four cases with time. 

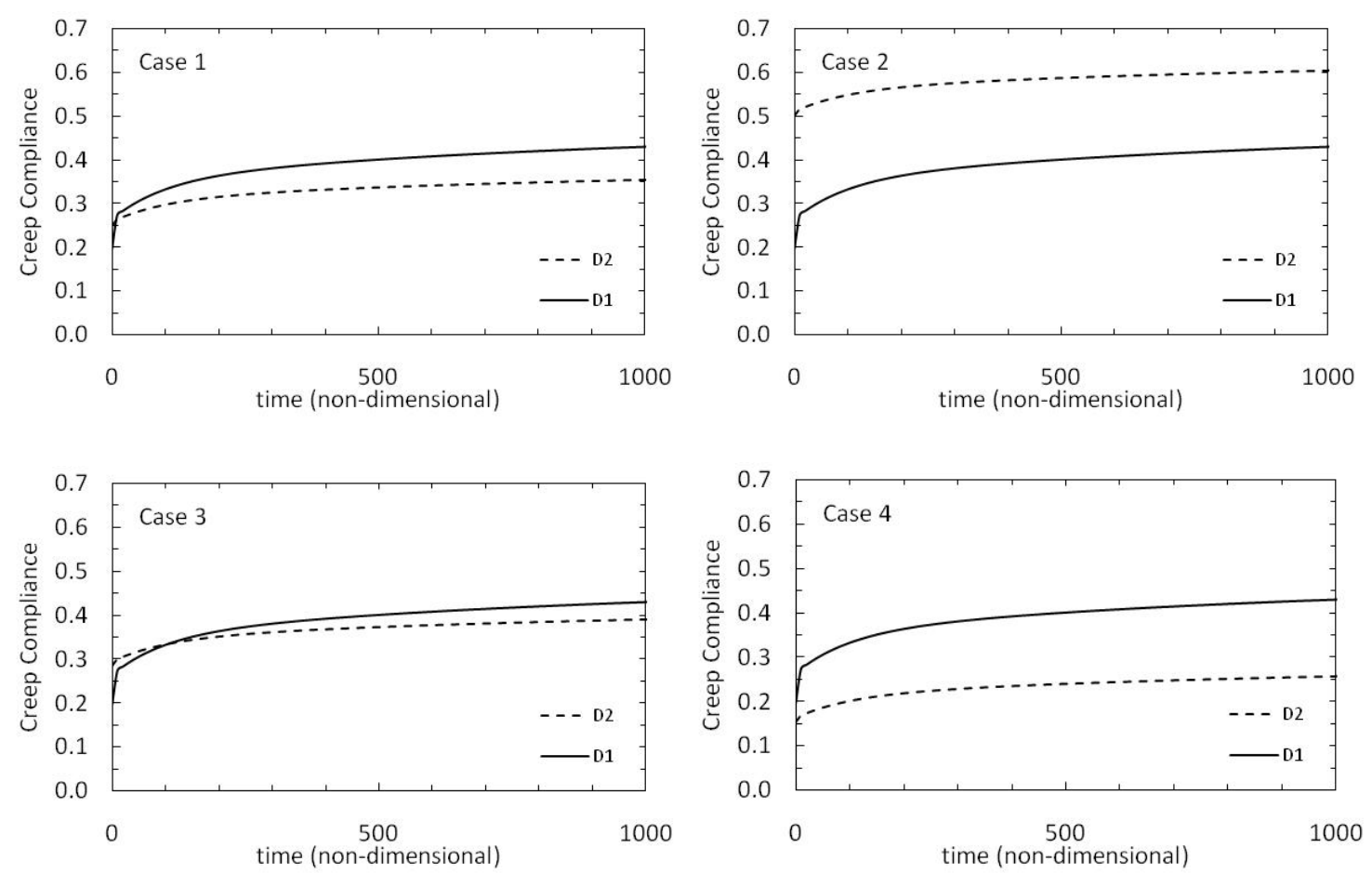

Fig. 4.9. Creep compliance of layer 1 and layer 2 for all the four cases with time

The material properties of layer 1 and layer 2 in all four cases as stated in the Table 4.3 and 4.4 are made temperature dependent in a similar manner as discussed in chapter III. The material properties are assumed to have linear dependence on temperature given by Eq.s (3.6) and (3.7).

Fig. 4.10 shows the hoop stress in the body at different times for all the four cases. At time $t=0$ the maximum value of the jump at the interface of $r=2.0$ is observed in case 2 as shown in Fig. 4.10.a, i.e. the jump at the interface in case 2 is approximately $350 \%$ higher compared to case $1,154 \%$ compared to case 3 and $48 \%$ compared to case 4 . This is as expected as in case 2 the amount of mismatch in the elastic moduli of the two 
layers is the most compared to all other cases. It is to be noted that the jump in the hoop stresses at $\mathrm{r}=2.0$ in the case 1 , case 2 and case 3 decreases with the increasing time whereas in case 4 , the amount of jump is increases as time progresses. At time $\mathrm{t}=1000$, the jump at the interface in the case 1 is less by $95 \%$ as compared to case 2 , the jump in case 3 is less by $82 \%$ when compared to case 2 and the jump in case 4 is less by $9 \%$ as compared to case 2. It is concluded that the effect of change in the instantaneous part of the compliance on the hoop stress is pronounced at the initial times as well as at longer times.
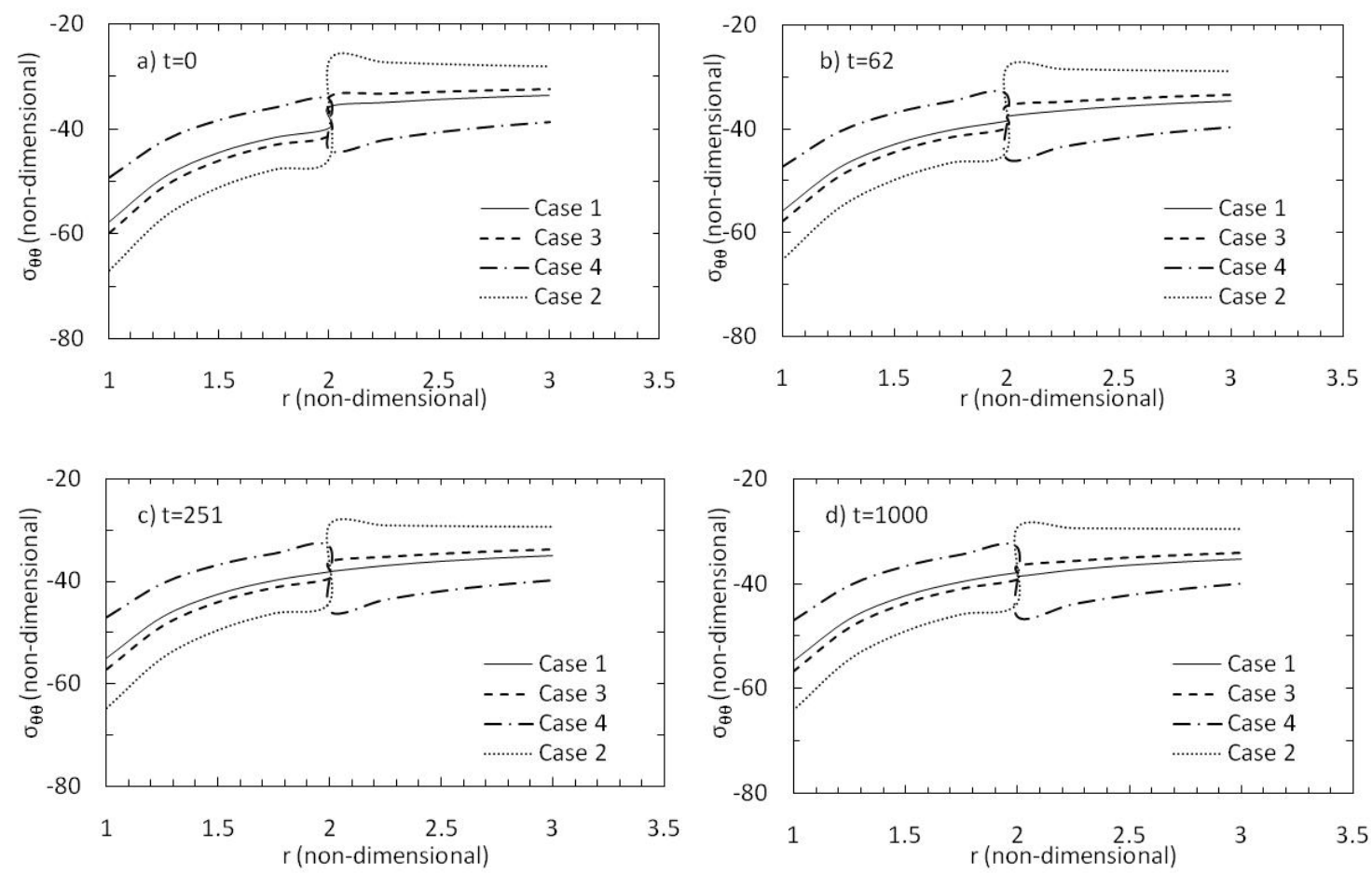

Fig. 4.10. Hoop stress for the composite cylinder at different times for different elastic part of compliance 
Fig. 4.11 shows the radial strain developed in the body at different times for all four cases. The jump in the radial strain at the interface of $r=2.0$ is higher in case 2 as compared to case 1 , case 3 and case 4 . This is expected as in case 2 the amount of mismatch in the elastic moduli of the two layers is the most compared to all other cases. It is noted that the jump in the radial strain at the interface increases as time advances in case 1 , case 2 and case 4 , whereas in case 3 as time increases, the magnitude of the jump decreases. At $\mathrm{t}=1000$, there is almost negligible jump at the interface for case 3 as seen in Fig. 4.11.d. At $\mathrm{t}=1000$, the maximum jump occurs in case 2, whereas the jump in case 1 is less by $87 \%$ as compared to case 2 and jump in case 4 is less by $54 \%$ as compared to case 2.
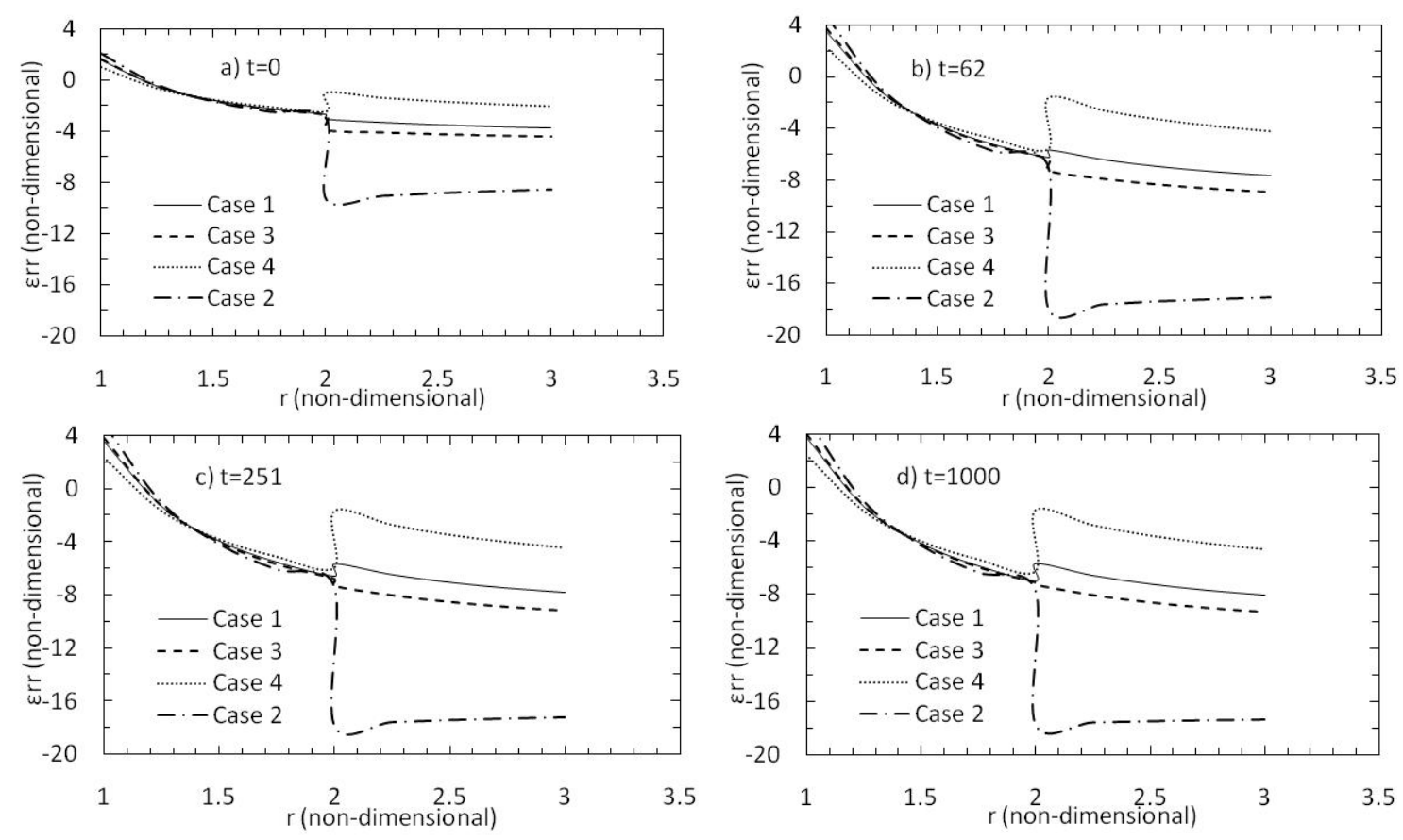

Fig. 4.11. Radial strain for the composite cylinder at different times for different elastic part of compliance 
Fig. 4.12.a shows the radial displacement at time $\mathrm{t}=0$ of the composite cylinder. The radial displacement at $\mathrm{r}=3.0$ at time $\mathrm{t}=0$ is maximum in case 2 , which is $48 \%$ higher as compared to case 1 , almost $38 \%$ higher as compared to case 3 and approximately $94 \%$ increase compared to case 4. As the time progresses, the value of radial displacement goes on increasing for all the four cases as seen from the Fig. 4.12. It can be seen from the Fig. 4.12.d, that at time $\mathrm{t}=1000$ the radial displacement in case 2 is $43 \%$ higher as compared to case $1,34 \%$ higher as compared to case 3 and about $80 \%$ higher when compared to case 4 . It is observed that the effect of change in the elastic part of compliance has significant effect on the radial displacement field of the body.
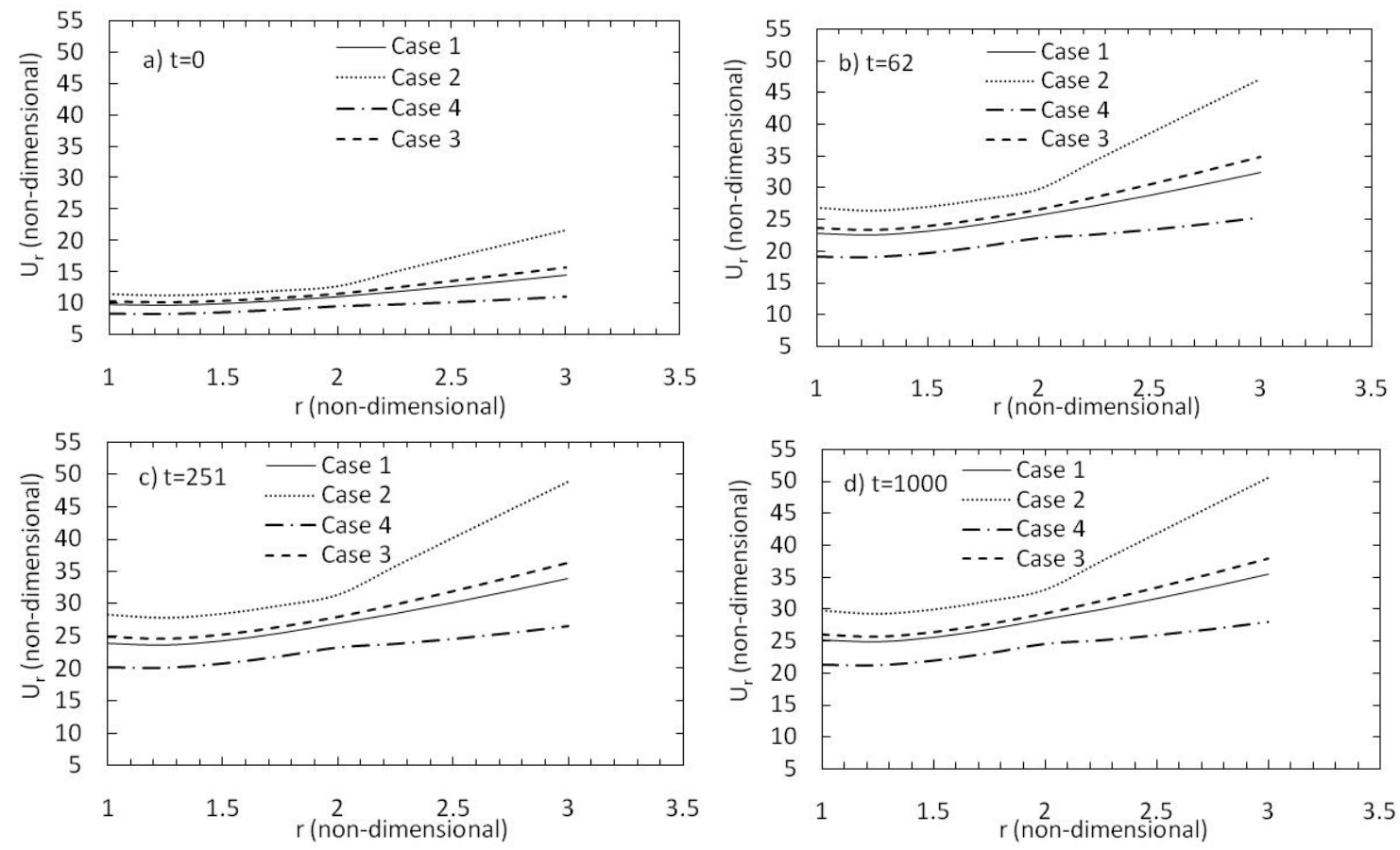

Fig. 4.12. Radial displacement for the composite cylinder at different times for different elastic part of compliance 
As seen from the Fig. 4.10, the jump at the interface at $r=2.0$ in hoop stress is minimum in case 1 compared to other cases at all the times. It should however be noted that the jump in the radial strain at the interface at $r=2.0$ is minimum in case 1 at initial times upto $t=62$, but is higher at the longer times when compared to case 3 . However the increase in the jump at the interface of $r=2.0$ in case 1 at higher times is not much compared to the case 2 and case 4 . Also, the radial displacement is minimum in case 4 at all the times but the jump at the interface at $\mathrm{r}=2.0$ in radial strain and hoop stress is maximum. Thus it can be concluded that the material properties of the two layers as considered in case 1 is best suited to avoid debonding caused due to higher stress discontinuity.

\subsection{EFFECT OF TEMPERATURE DEPENDENT FUNCTIONS ON THE OVERALL RESPONSE OF THE COMPOSITE CYLINDER}

The studies that are carried out till now investigates the effect of varying the elastic and time-dependent material parameters on the overall response. It was considered that both layers of the composite cylinder have same form of temperature dependence that was assumed to be linear. The present study aims at understanding the effect of different forms of the temperature dependence in the two layers the materials on the overall material response. The time dependent compliances for the two layers are given in chapter III. Two different studies are carried out as described below. 


\subsubsection{LINEAR FORM OF TEMPERATURE DEPENDENT MATERIAL PROPERTIES}

The material properties of the two layers of the composite cylinder are considered to have linear dependence on temperature. Two different cases are considered. In case 1, the material properties of layer 1 and layer 2 are assumed to have different temperature dependent linear functions. Case 2 has the identical temperature dependent linear functions for the layer 1 and layer 2 as discussed in chapter III. The aim is to analyze the effect of different temperature dependent functions for two layers on the overall response of the composite cylinder. In the case 1, the temperature dependent functions for layer 1 and layer 2 are:

$$
\begin{aligned}
& D_{o}^{1}(T)=D_{o}^{1}(1+T) \\
& D_{n}^{1}(T, t)=D_{n}^{1}(0.5 T)\left(1-e^{\left(-\lambda_{n} \xi(t)\right)}\right) \\
& D_{o}^{2}(T)=D_{o}^{2}(1+1.5 T) \\
& D_{n}^{2}(T, t)=D_{n}^{2}(0.75 T)\left(1-e^{\left(-\lambda_{n} \xi(t)\right)}\right)
\end{aligned}
$$

where $\xi(t)=\int_{0}^{t} \frac{d s}{a\left(T(s), T_{o}\right)}$ is the reduced time with

$a\left(T, T_{o}\right)=\frac{1}{1+\left(T-T_{o}\right)}$; where $\mathrm{T}_{\mathrm{o}}$ is the reference temperature considered to be 0 in this study since non dimensional quantities are considered in this study. $D_{o}^{1}$ and $D_{o}^{2}$ denote the elastic part of compliances of the layer 1 and layer 2, respectively. $D_{n}^{1}$ and $D_{n}^{2}$ denote the $\mathrm{n}^{\text {th }}$ coefficient of the transient part of creep compliances of layer 1and layer 2 , 
respectively. In case 2 , the material properties of the two layers are assumed to have the same linear temperature dependent function as given in the Eq.s (3.6) and (3.7) which are mentioned below:

$D_{o}^{(i)}(T)=D_{o}^{(i)}(1+T)$

$D_{n}^{(i)}(T, t)=D_{n}^{(i)}(0.5 T)\left(1-e^{\left(-\lambda_{n} \xi(t)\right)}\right) \quad \mathrm{i}=1,2$ corresponds to layers 1 and 2

where $\xi(t)=\int_{0}^{t} \frac{d s}{a\left(T(s), T_{o}\right)}$ is the reduced time with $a\left(T, T_{o}\right)=\frac{1}{1+\left(T-T_{o}\right)}$

where $T_{0}$ is the reference temperature considered to be 0 in this study since non dimensional quantities are considered in this study. Table 4.5 shows the elastic and transient material parameters of the creep compliance of layer 1 and layer 2 . These material properties are the same for the case 1 and case 2 :

Table 4.5. Elastic properties and transient properties of the creep compliance for the material of layer 1 and layer 2 which are same for case 1 and case 2

\begin{tabular}{|c|c|c|c|c|c|}
\hline \multicolumn{3}{|c|}{ Layer 1 } & \multicolumn{3}{c|}{ Layer 2 } \\
\hline $\mathrm{n}$ & $\lambda_{\mathrm{n}}$ & $\mathrm{D}_{\mathrm{n}}$ & $\mathrm{n}$ & $\lambda_{\mathrm{n}}$ & $\mathrm{D}_{\mathrm{n}}$ \\
\hline & & & & & \\
1 & 1 & 0.07 & 1 & 1 & 0.01 \\
2 & 0.01 & 0.09 & 2 & 0.01 & 0.05 \\
3 & 0.001 & 0.12 & 3 & 0.001 & 0.07 \\
\hline \multicolumn{3}{|c|}{} & & & \\
\end{tabular}


Fig. 4.13 shows the hoop stress in the composite cylinder at different times for both cases. As seen in the Fig. 4.13.a, at $\mathrm{t}=2.0$, the magnitude of the jump in the hoop stress at the interface at $\mathrm{r}=2.0$ in case 1 is higher by almost $200 \%$ as compared to case 2 . As the time increases, the magnitude of jump decreases in both cases. As seen in Fig. 4.13.c, the amount of jump at the interface in case 2 is almost negligible as compared to the jump of 6.12 units in case 1 . At time $t=1000$, the jump in case 1 is 5.7 units as compared to 0.19 units in case 2 . It is worth noting that the jump at the interface increases significantly when layer 1 and layer 2 have different linear temperature dependent functions.
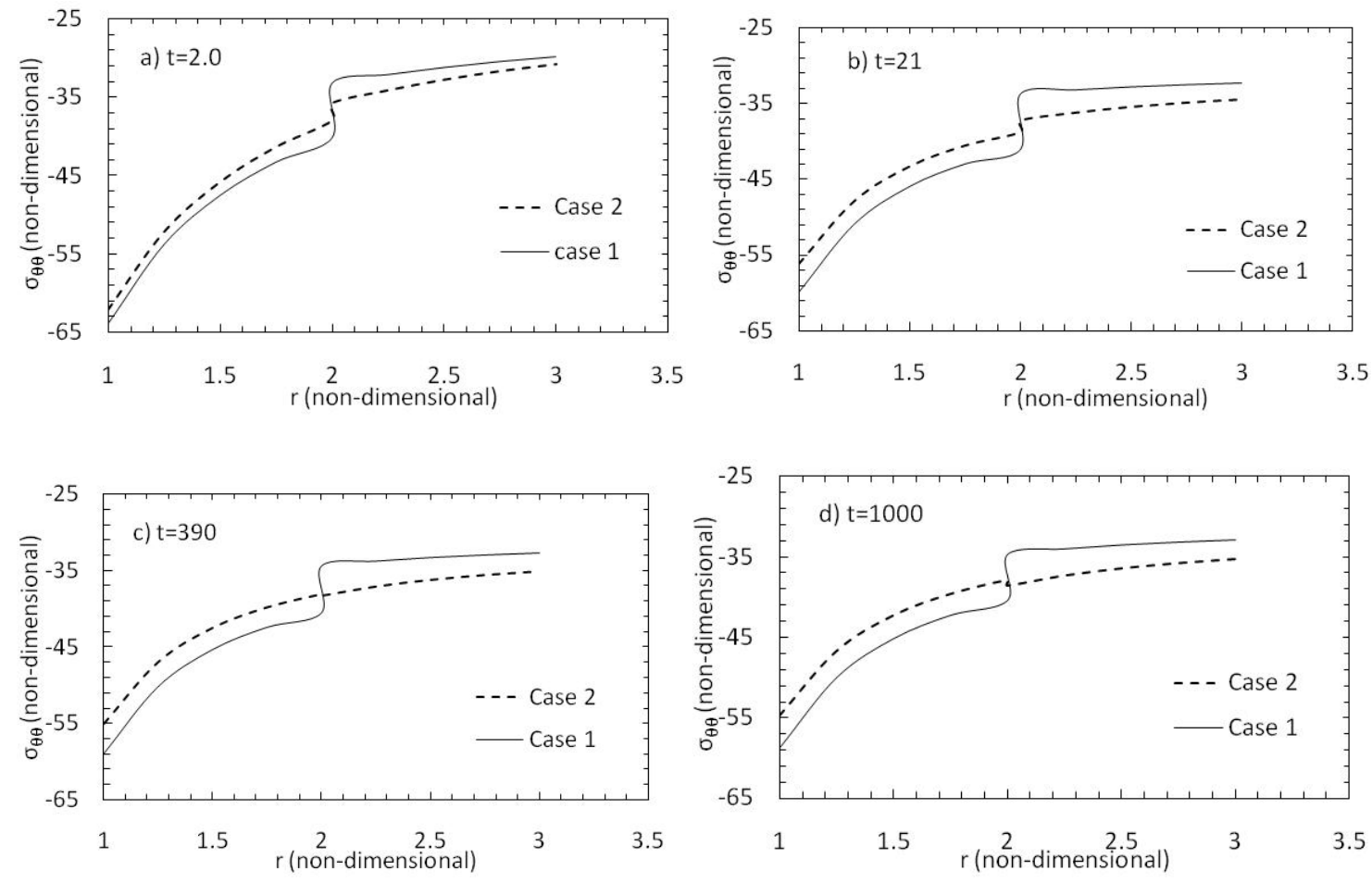

Fig. 4.13. Hoop stress for the composite cylinder at different times for different temperature dependence of two layers 
Fig. 4.14 shows radial strain in the composite cylinder for both the cases at different times. As seen from the Fig. 4.14.a, at $t=2$, the magnitude of the jump at the interface at $\mathrm{r}=2.0$, is almost negligible in case 2 whereas in case 1 it is as high as 2.3 units. With increase in the time, the jump in the radial strain at the interface increases for the case 2, while for the case 1 it increases upto $t=21$, and then decreases with the increasing time. At time $\mathrm{t}=1000$, the magnitude of the jump in case 1 is higher by approximately $88 \%$ as compared to case 2 . Thus a significant effect is observed on the radial strains when the two layers have different linear temperature dependent functions.
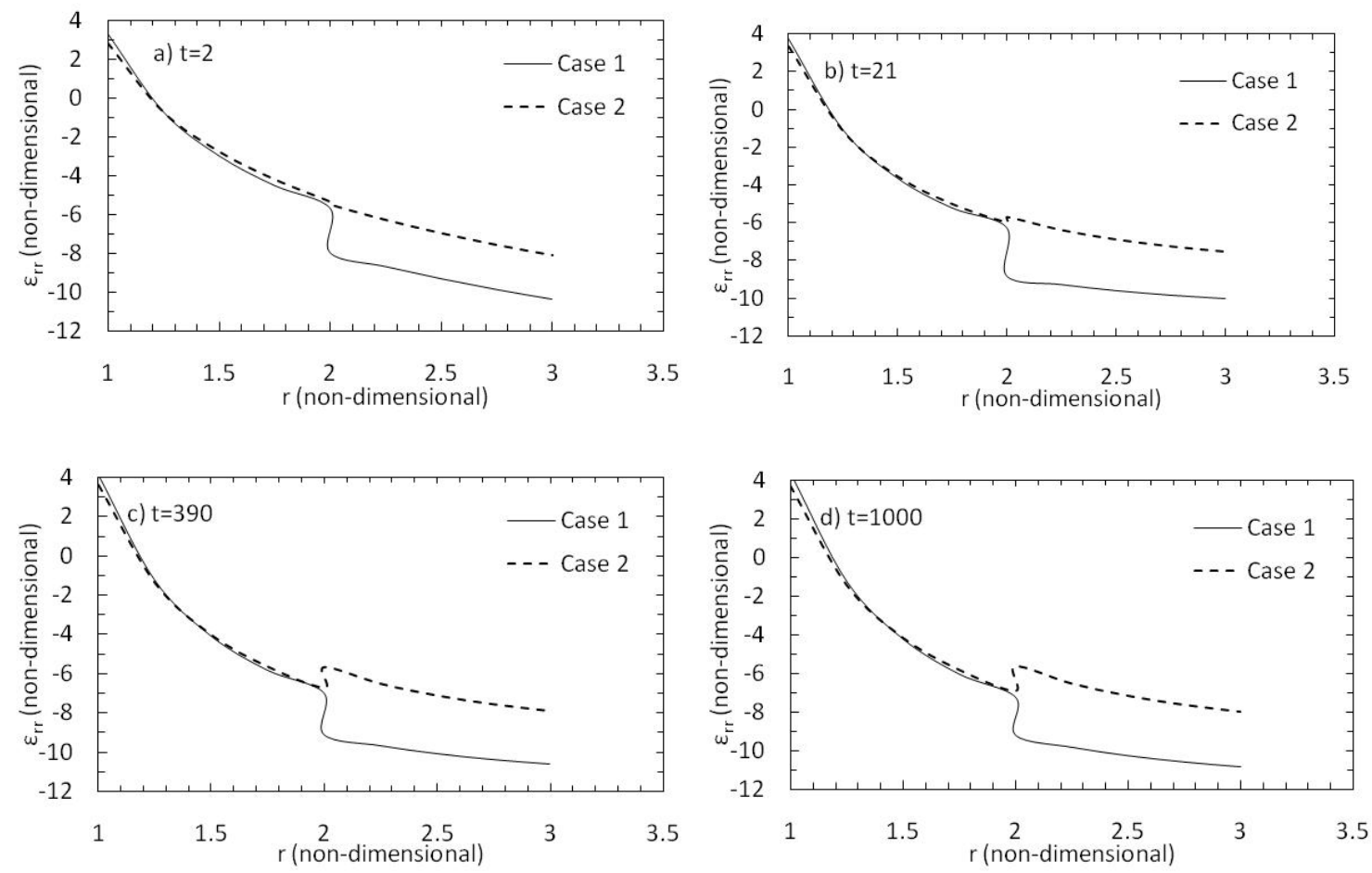

Fig. 4.14. Hoop strain for the composite cylinder at different times for different temperature dependence of two layers 
As seen in the Fig. 4.15.a, the radial displacement in the case 1 at $r=3.0$ is $18 \%$ higher as compared to the one in case 2. The magnitude of the radial displacement increases as the time progresses in both cases. At $\mathrm{t}=21$, insignificant difference is observed in the values of radial displacement in the two cases as seen in the Fig. 4.15.b. With the increase in time, the magnitude of the radial displacement is higher in case 1 as compared to case 2 as seen in the Figs $4.15 \mathrm{c}$ and 4.15.d. At time $\mathrm{t}=1000$, the radial displacement at $r=3.0$ in case 1 is $13 \%$ higher as compared to case 2 . Thus overall response of the composite cylinder is strongly dependent on temperature functions.
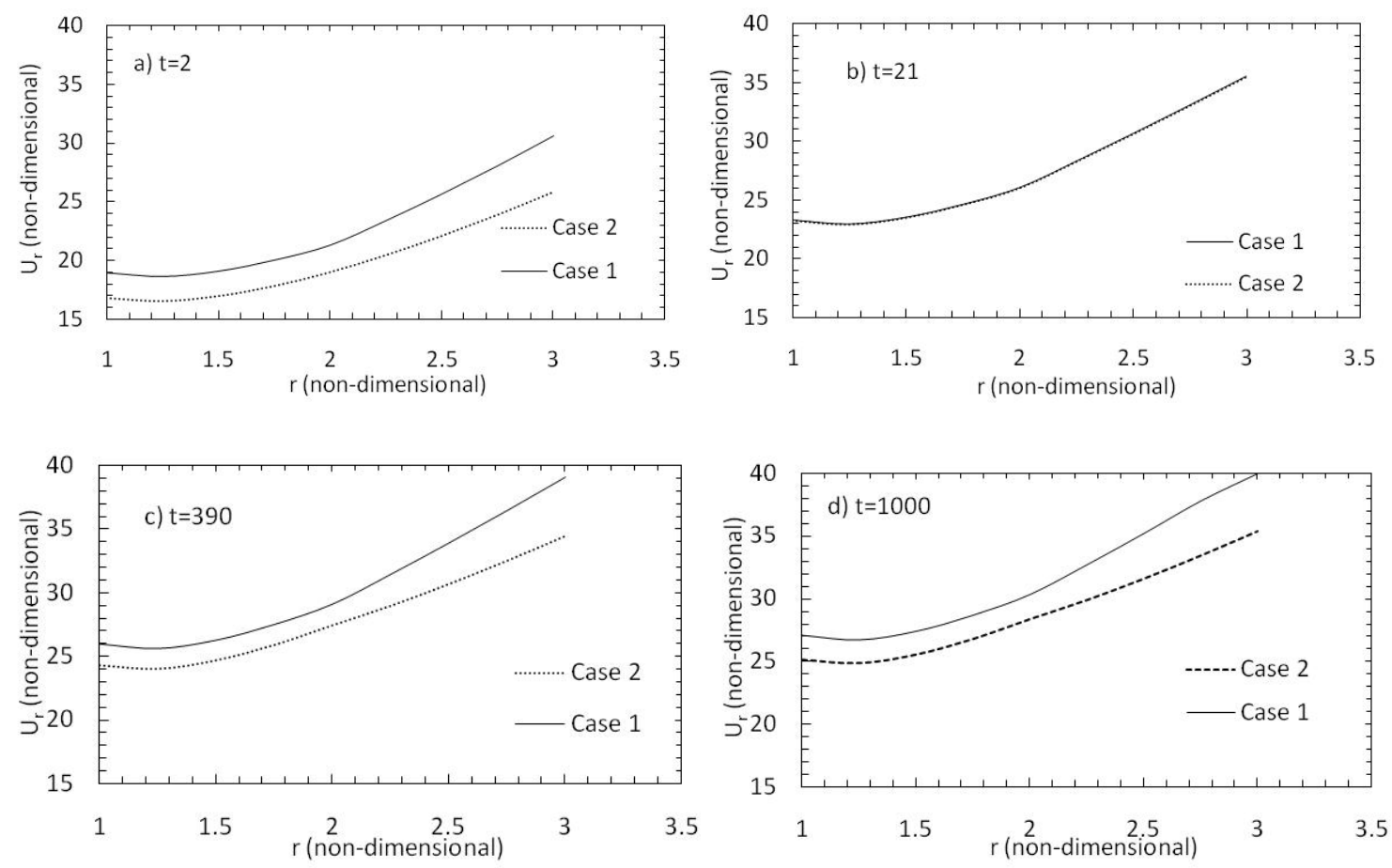

Fig. 4.15. Radial displacement for the composite cylinder at different times for different temperature dependence of two layers 


\subsubsection{QUADRATIC POLYNOMIAL FORM OF TEMPERATURE DEPENDENCE OF MATERIAL PROPERTIES}

In the previous study, material properties were assumed to have linear dependence of temperature. In this final study, the material properties (elastic part of creep compliance) of both layers are assumed to have quadratic polynomial form of temperature dependence. This is mainly done to illustrate the effect of stronger temperature dependence on the material properties and the subsequent effect on the overall response of the composite cylinder. Two different cases are considered. In case 1, the material properties of layer 1 and layer 2 are considered to have same quadratic polynomial form of temperature dependence given by:

$$
D_{o}^{(i)}(T)=D_{o}^{(i)}\left(1+T+T^{2}\right)
$$

$D_{n}^{(i)}(T, t)=D_{n}^{(i)}(0.5 T)\left(1-e^{\left(-\lambda_{n} \xi(t)\right)}\right), \mathrm{i}=1,2$ corresponds to layers 1 and 2

where $\xi(t)=\int_{0}^{t} \frac{d s}{a\left(T(s), T_{o}\right)}$ is the reduced time with

$$
a\left(T, T_{o}\right)=\frac{1}{1+\left(T-T_{o}\right)}
$$

where $T_{0}$ is the reference temperature considered to be 0 in this study since non dimensional quantities are considered in this study. $D_{o}^{(i)}$ and $D_{n}^{(i)}$ denotes the elastic part and transient part of creep compliance of $i^{\text {th }}$ layer respectively.

In case 2 , the material properties of both layers are assumed to have linear form of temperature variation given by the Eq. (4.2). These two cases, one with quadratic polynomial form of temperature dependent material properties and the other with linear 
dependence on temperature of material properties, are considered so that the effect of the form of temperature dependence can be studied. The elastic properties and the transient creep parameters of both the layers are taken as mentioned in the Table 4.5.

Fig. 4.16 shows the hoop stress in the body for both cases at different times. As seen from Fig. 4.16.a, at $\mathrm{t}=2$, the jump at the interface at $\mathrm{r}=2.0$ in case 1 is $9 \%$ higher than case 2. With the increasing time, the magnitude of jump at the interface at $r=2.0$ decreases for the case 1 whereas in case 2 , it decreases upto certain time and then again back increases at longer time. As seen from the Fig. 4.16.c, at $t=390$, there is almost
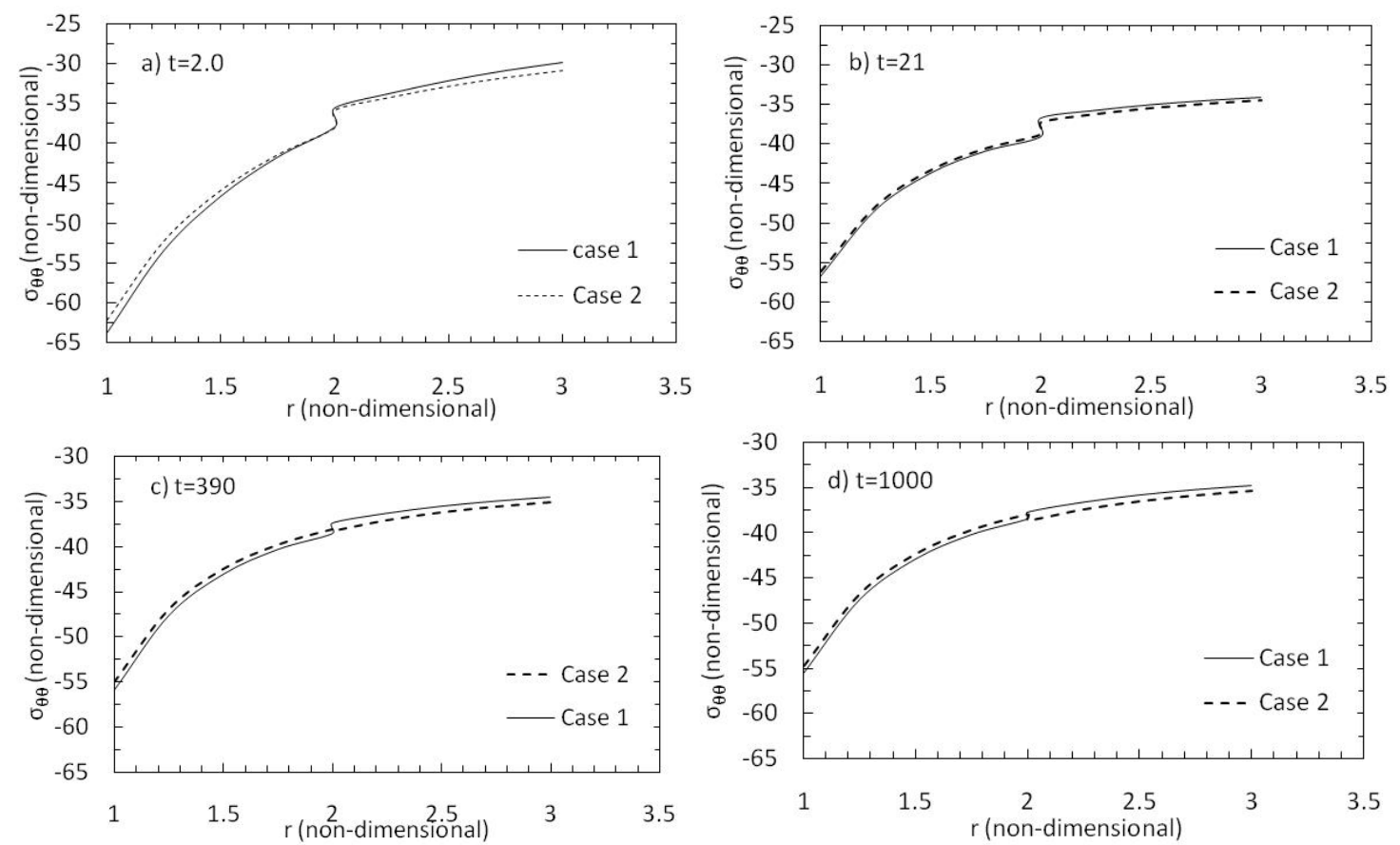

Fig. 4.16. Hoop stress for the composite cylinder at different times for different temperature dependence (quadratic) of two layers 
negligible jump at the interface in case 2, whereas the jump in case 1 is around 1.16 units. As seen from the Fig. 4.16.d, at $\mathrm{t}=1000$ the jump at $\mathrm{r}=2.0$ in the case 1 is approximately $12.5 \%$ higher than in case 2 . It is worth noting that the effect of quadratic form of temperature dependence on the material properties as compared to linear form of temperature dependence is significant on hoop stress at all the times.

As seen from Fig. 4.17.a, the magnitude of the radial strain in the case 1 is higher compared to case 2 at initial times at $t=2.0$. The magnitude of the radial strain at $r=3.0$ at $\mathrm{t}=2.0$ in case 1 is approximately $53 \%$ more than case 2 . Also the jump at the interface at $\mathrm{r}=2.0$ in case 1 is almost $150 \%$ more than the jump in case 2 . This shows that the effect of strong temperature dependence on the material properties is significant on the radial strains at initial times. As the time increases, it is seen that the jump at the interface at $\mathrm{r}=2.0$ in case 2 goes on increasing while in case 1 , the jump decreases upto certain time and then again increases. At $\mathrm{t}=1000$, the magnitude of the jump at the interface at $\mathrm{r}=2.0$ in case 2 is almost $10.5 \%$ higher as compared to case 1 , but the actual magnitude of the radial strain are much higher in case 1 as compared to case 2 . At $\mathrm{t}=1000$, the actual magnitude of radial strain at $r=3.0$ in case 1 is higher by approximately $46 \%$ when compared to case 2 . It can be concluded that when the material properties of the two layers are strongly dependent on temperature, higher radial strain values are obtained at all the times. 

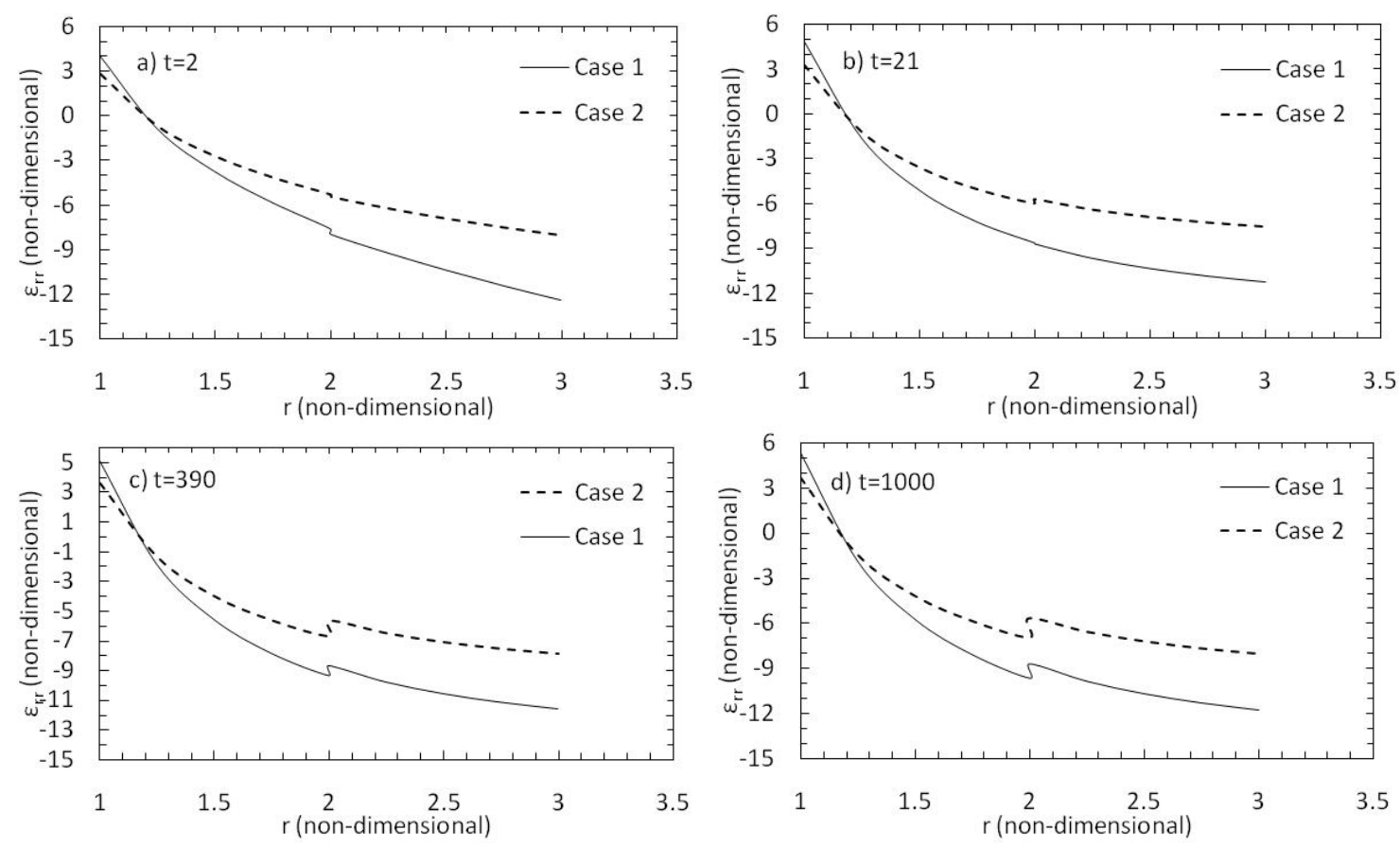

Fig. 4.17. Hoop strain for the composite cylinder at different times for different temperature dependence (quadratic) of two layers

As shown in the Fig. 4.18.a, the radial displacement at initial times at $\mathrm{t}=2.0$ is much higher in case 1 as compared to case 2 . The radial displacement at $r=3.0$ in case 1 at $\mathrm{t}=2.0$ is approximately $40 \%$ higher than case 2 . As seen from the Fig. 4.18.b, insignificant difference is seen in the values of radial displacement at time $t=21$. As the time progresses further, the radial displacement in the case 1 is much higher compared to case 2 as seen from the Fig. 4.18.c and 4.18.d. At time $\mathrm{t}=1000$, the radial displacement at $r=3.0$ in case 1 is $41 \%$ higher as compared to case 2 . Thus the effect of strongly temperature dependent material properties on the radial displacement is quite significant at longer times. 

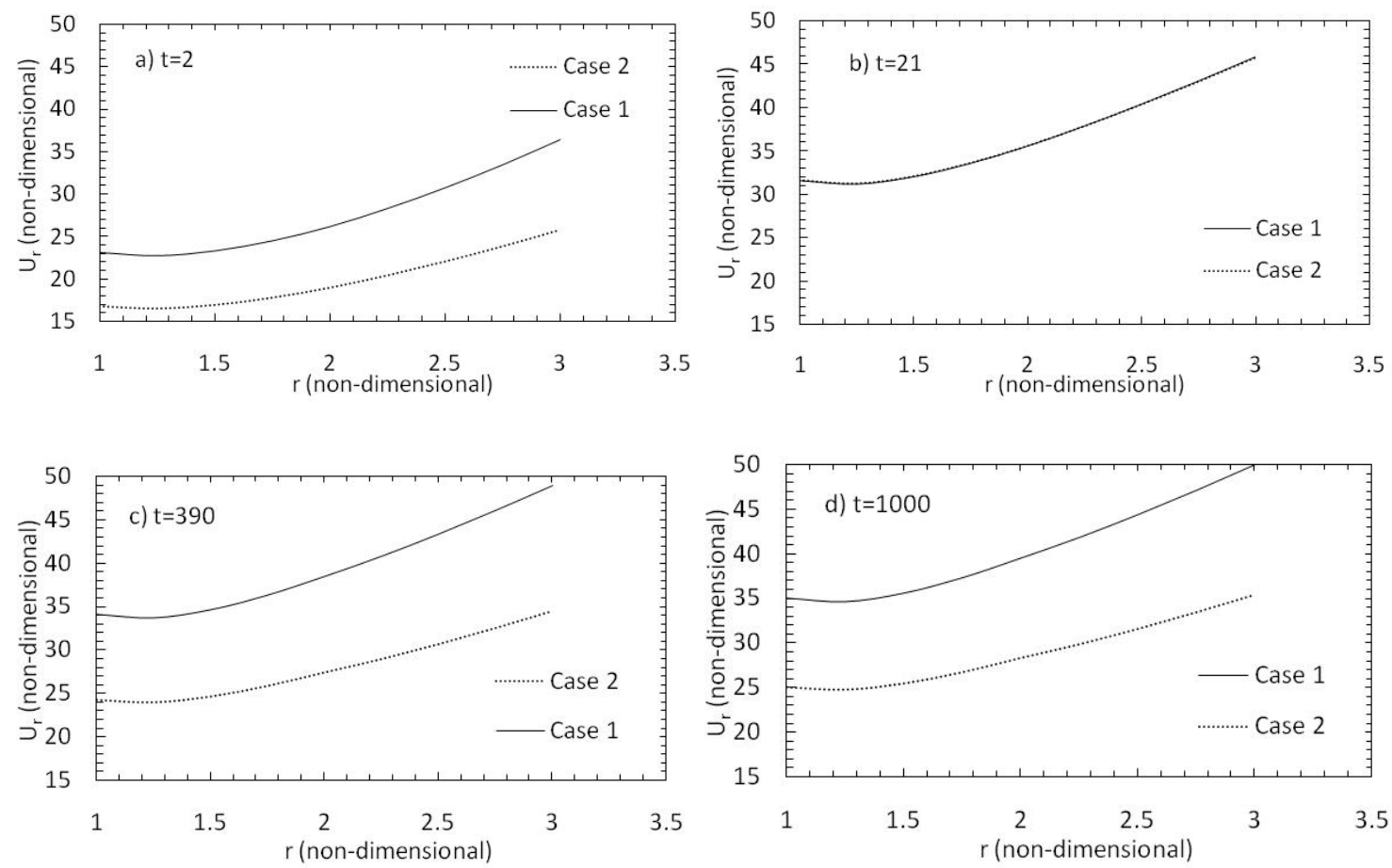

Fig. 4.18. Radial displacement for the composite cylinder at different times for different temperature dependence (quadratic) of two layers

As seen from Fig. 4.16, the jump at the interface at $r=2.0$ in hoop stress is minimum in case 2 as compared to case 1 at all the times upto $t=1000$. Also, the jump at the interface at $\mathrm{r}=2.0$ in radial strain is almost negligible in case 2 during initial times upto $t=21$. At longer times, the jump in the radial strain at $r=2.0$ in case 2 is almost same as compared to case 1 . In case 2 the radial displacement is much lower compared to the one in case 1 as seen from the Fig. 4.18. It can thus be concluded that the materials having linear temperature dependent functions or in other words, materials that are not so strongly dependent on temperature, as assumed in case 2 are best suited from design point of view where the jump in the radial strain and hoop stress is not desired. These 
results that highlight the characteristics of the jump in the hoop stress and radial strain at the interface at $\mathrm{r}=2.0$ can be used as guidelines while doing material selection from design point of view so as to minimize the jump and ensure sufficient bonding at the interface at all the times.

\subsection{OBSERVATIONS}

The parametric studies done in the above sections by varying the material properties (elastic and transient) in first three sections and varying the temperature dependent functions in the last section shows the effect of these properties on the overall response of the composite cylinder. It is observed that on assuming identical temperature dependent functions, the effect of varying the characteristics of creep time of transient compliance as seen from the Fig. 4.6-4.8, on the overall response is insignificant compared to the effect of varying other material properties (elastic part and Prony coefficients). As seen above, there arises a discontinuity in the hoop stress and radial strain at the interface of two layers due to mismatch in material properties of two layers. If this value of the jump exceeds a certain value, then it may lead to delamination of the two layers which is generally not desired in many design applications. Also, some applications require the maximum deformation of the body to be within certain limit. Hence the mechanical response in terms of hoop stress, radial strain and radial displacement is studied in all the studies so that these characteristics can be used while design.

The effect of different Prony coefficients of the layer 2 on the overall response of the composite cylinder, as seen from the Fig. 4.2-4.4 is not that significant during the 
initial times but is seen to be significant at the longer times. In all three cases considered in section 4.1, during initial times, the difference in the overall value of creep compliance of two layers is almost the same but as time progresses, the difference in the values of creep compliance increases. This is reflected in the overall response, which is almost the same in all three cases during initial times but as time progresses, significant effect is observed in the overall response in all three cases.

The effect of different elastic part of compliance is significant on the overall response. As discussed in section 4.3, when the ratio of elastic part of compliance of layer 1 to that of layer 2 is changed from 1.282 in case 1 to 2.564 in case 2 and to 0.788 in case 4 , the overall responses are significantly altered at initial as well as longer times where as when the ratio is slightly changed to 1.46 in case 3 , the effect on the overall response is observed to be not so pronounced. This is as expected as higher mismatch in the elastic part causes a higher difference in the material compliance values at initial as well as longer times as seen from the Fig. 4.9, which causes higher jump discontinuities in hoop stress as well as radial strain. Also, it is to be noted that when the layer 1 is stiffer as compared to layer 2 as in case 2 where the ratio of elastic part of compliance of layer 1 to that of layer 2 is 2.564 , the jump in the hoop stress decreases with increase in time. Whereas when the layer 2 is stiffer compared to layer 1 like in case 4 , where the ratio of elastic part of compliance of layer 1 to layer 2 is 0.788 , the jump in the hoop stress goes on increasing with time. It is also observed that higher mismatch in elastic part of compliance causes a pronounced increase in the value of overall radial 
displacement (deformations) developed in the composite body as seen from the Fig. 4.12 as compared to Fig. 4.4 or Fig. 4.8 which shows the effect of change in transient part.

In the last parametric study, the material properties of the two layers are kept constant while the effect of temperature dependent functions is investigated. It is observed that when the material properties are made strongly dependent on temperature, the overall responses are altered significantly as seen from the Fig. 4.16-4.18. As seen from the Fig. 4.18, the overall deformations at longer times $t=1000$ increases significantly when the strongly temperature dependent functions are considered.

The above mentioned results that highlight the effect of varying material properties and temperature dependent functions on the overall response of the composite cylinder can be used for material selection criteria. It should be noted that to avoid the debonding at the interface due to high discontinuity in the hoop stress and radial strain, the materials selected for the two layers should not have a high mismatch in the elastic part of compliance. Also one should bear in mind that if the materials of the two layers have strongly dependent temperature functions then the chances of high discontinuities in the stress will be high that may lead to debonding at the interface at higher times. Also if the mismatch in the transient part of the creep compliance of the two layers is high, it may increase the jump at the interface in the hoop stress and radial strain at the longer times. Thus to avoid the debonding at the interface due to jump discontinuities materials that donot have higher mismatch in the overall creep compliance values at all the times should be preferred. 


\section{CHAPTER V}

\section{CONCLUSIONS AND FURTHER RESEARCH}

\subsection{CONCLUSIONS}

This study analyzes response of the composite cylinder consisting of two layers of linear isotropic viscoelastic materials belonging to a class of non-TSM, subjected to thermo-mechanical loading. Sequentially coupled analysis of heat conduction and deformation of viscoelastic composite cylinder is carried out. The temperature effects are incorporated by considering the material properties of the two layers at a particular location at a given instant of time to depend on the instantaneous value of temperature at that location. The analytical solution for determining the stress, strain and displacement fields developed in the composite cylinder when subjected to mechanical loads is obtained from the corresponding solution of linear elasticity problem by using the Correspondence Principle. The analytical solution for determining the temperature dependent stress, strain and displacement fields is developed by incorporating the temperature dependence on the material properties and modeling the material as nonTSM. To analyze more complex geometries with general loading and boundary conditions, FE analysis of the composite cylinder is performed. Convergence study is carried out to ensure that the results obtained from the FE model are adequately accurate and the results of analytical and FE method are in good agreement. The effect of temperature dependent material properties as compared to the temperature independent material properties is found to be significant on the overall response of the composite cylinder in terms of stress, strain and displacement fields. Various parametric studies 
have been carried out to understand the effect of varying material parameters and the temperature dependent functions on the overall response. Studies have been carried out to understand the effect of change in material parameters namely the Prony coefficients in the transient creep compliance, characteristic of creep time of transient creep compliance and the instantaneous (elastic) compliance on the overall response of the composite cylinder. It is observed that the change in elastic compliance and the Prony coefficients has a pronounced effect on the overall response of the composite cylinder whereas the effect of change in the characteristic of the creep time is less significant. In the last section the effect of different temperature dependent functions of the material properties, namely linear temperature variation and quadratic polynomial variation is analyzed on the overall material response. It is observed that the stronger dependence on temperature (quadratic polynomial) of the material properties has a significant effect on the overall stress, strain and displacement fields developed. The results from the parametric studies provide useful guidelines for material selection in the design aspects where the discontinuity (jump) at the interface in hoop stress and radial strain is not desired. It is worth noting that the materials having considerable mismatch in the elastic properties of the two layers can cause a significant increase in the jump at the interface in radial strain and hoop stress. Also, the materials that have a strong dependence on the temperature (quadratic variation) can cause higher values of jump at the interface in hoop stresses and higher radial displacement. The results describing the effect of material parameters and the temperature dependence can thus help in deciding the design 
criterion to minimize the jump at the interface so as to ensure sufficient bonding at the interface at all the times.

\subsection{FURTHER RESEARCH}

The present study analyses the response of composite cylinder made up of linear viscoelastic material whose properties are made temperature dependent. This study can be extended to analyze the response of the composite cylinder made up of non-linear viscoelastic solid materials subjected to thermo-mechanical loadings. The stress and temperature dependent material properties should be then incorporated in the constitutive relation to obtain the stress and strain fields. This type of study can also be extended to analyze the response of composite cylinder made up of non-linear elastic materials subjected to temperature or moisture loadings. The study can as well be extended to analyze the response of the composite cylinder made up of anisotropic materials. Also, in the present study a coupled problem of heat conduction and deformation is considered where the deformation of the composite depends on the temperature but the temperature profile is obtained without knowledge of stress or strain. A fully coupled problem can be analyzed where the evolution of temperature in the body with time depends on the stress or strain fields and also the deformation of the body depends on the temperature. The viscoelastic materials dissipate energy. This dissipated energy is in form of heat which can cause an increase in temperature if the amount of dissipation is considerable. The change in material properties due to this increase in temperature should be accounted for to evaluate the overall response in a fully coupled problem. 


\section{REFERENCES}

[1] Alfrey T. Non-homogeneous Stresses in Viscoelastic Media. Quaterly of Applied Mathematics 1944;2:113-119.

[2] Lee EH, Radok JRM, Woodward WB. Stress Analysis for Linear Viscoelastic Materials. Transactions of the Society of Rheology 1959; III:41-59.

[3] Gurtin ME, Sternberg E. On the Linear Theory of Viscoelasticity. Archive for Rational Mechanics and Analysis 1962;11:291-356.

[4] Lee EH, Rogers TG. Solution of Viscoelastic Stress Analysis Problems Using Measured Creep or Relaxation Functions. Journal of Applied Mechanics 1963;30:127-133.

[5] Rajagopal KR, Wineman AS. A Quasi-Correspondence Principle for Quasi-Linear Viscoelastic Solids. Mechanics of Time-Dependent Materials 2008;121-14.

[6] Rajagopal KR, Wineman AS. Mechanical Response of Polymers:An Introduction. New York:Cambridge University Press, 2000.

[7] Rajagopal KR, Srinivasa AR. A Note on a Correspondence Principle in Nonlinear Viscoelastic Materials. International Journal of Fracture 2005;131:L47-L52.

[8] Schapery RA. Correspondence Principles and Generalized J Integral for Large Deformation and Fracture Analysis of Viscoelastic Media. International Journal of Fracture 1984;25(3):195-223.

[9] Rajagopal KR and Wineman AS. A Note on Viscoelastic Materials that can Age. International Journal of Non-Linear Mechanics 2004;39:1547-1554.

[10] Rajagopal KR, Kannan K and Rao IJ. A Thermomechanical Framework for the Glass Transition Phenomenon in Certain Polymers and its Application to Fiber Spinning. Journal of Rheology 2002;46:977-999. 
[11] Rajagopal KR and Kannan K. A Thermomechanical Framework for the Transition of a Viscoelastic Liquid to a Viscoelastic Solid. Mathematics and Mechanics of Solids 2004;9:37-59.

[12] Leaderman H. Creep Properties of Filamenteous Materials and Other High Polymers. Washington:McGraw Hill, 1943.

[13] Schwarzl F, Staverman AJ. Time-Temperature Dependence of Linear Viscoelastic Behavior. Journal of Applied Physics 1952;23(8):838-843.

[14] Lee EH and Morland LW. Stress Analysis for Linear Viscoelastic Materials with Temperature Variation. Transactions of the Society of Rheology 1960;IV:233-263.

[15] Christensen RM and Naghdi PM. Linear Non-Isothermal Viscoelastic Solids. Acta Mechanica 1967;3:1-12.

[16] Tobolsky AV and Catsiff E. Elastoviscous Properties of Polyisobutylene (and Othr Amorphous Polymers) from Stress-Relaxation Studies. IX. A Summary of Results. Journal of Polymer Science 1956;XIX:111-121.

[17] Lai JSY, Findley WN. Creep of Polyurethane Under Varying Temperature for Nonlinear Uniaxial Stress. Transactions of the Society of Rheology 1973;17(1):6387.

[18] Ward IM and Onat ET. Non-linear Mechanical Behaviour of Oriented Polypropylene. Journal of Mechanics and Physics of Solids 1963;11:217-229.

[19] Tschoegl NW and Fesko DG. Time-Temperature Superposition in Thermorheologically Complex Materials. Journal of Polymer Science Part C 1971;35:51-69.

[20] Caruthers JM and Cohen RE. Consequences of Thermorheologically Complexity in Viscoelastic Materials. Rheological Acta 1980;19;606-613. 
[21] Weitsman Y. On the Thermoviscoelastic Characterization of Adhesives and Composites. Progress in Science and Engineering of Composites: Proceedings of the fourth International Conference on Composite Materials 1982;1:771-777.

[22] Harper BD, Weitsman Y. Characterization Method for a Class of Thermorheologically Complex Materials. Journal of Rheology 1985;29(1):49-66.

[23] Knauss WG, Zhu W. Nonlinearly Viscoelastic Behavior of Polycarbonate. I. Response under Pure Shear. Mechanics of Time-Dependent Materials 2002;6:231269.

[24] Zienkiewicz OC, Watson M and King IP. A Numerical Method of Viscoelastic Stress Analysis. International Journal of Mechanical Science 1968;10:807-827.

[25] Taylor RL, Pister KS, Goudreau GL. Thermomechanical Analysis of Viscoelastic Solids. International Journal for Numerical Methods in Engineering 1970;2:45-59.

[26] Feng WW. A Recurrence Formula for Viscoelastic Constitutive Equations. International Journal of Nonlinear Mechanics 1992;27(4):675-678.

[27] Henriksen M. Non-linear Viscoelastic Stress Analysis-A Finite Element Approach. Computers and Structures 1984;18(1):133-139.

[28] Lai J, Bakker A. 3-D Schapery Representation for Non-linear Viscoelasticity and Finite Element Implementation. Computational Mechanics 1996;18:182-191.

[29] Haj-Ali RM, Muliana AH. Numerical Finite Element Formulation of the Schapery Non-linear Viscoelastic Material Model. International Journal for Numerical Mehtods in Engineering 2004;59:25-45.

[30] Muliana A, Khan K. A Time-Integration Algorithm for Thermo-Rheologically Complex Polymers. Computational Materials Science 2008;41:576-588. 
[31] Christensen RM. Theory of Viscoelasticity: An Introduction. New York:Academic Press, 1971.

[32] Sadd MH. Elasticity: Theory, Applications, and Numerics. Amsterdam; Boston: Elsevier Butterworth Heinemann, 2005. 


\section{VITA}

Name: Sneha B. Shah

Address: Department of Mechanical Engineering, 3123 TAMU, College Station, TX, 77843

Email Address: snehashah@tamu.edu

Education: B.E, Mechanical Engineering, Gujarat University, India, 2005

M.S, Mechanical Engineering, Texas A\&M University, College

Station, Texas, 2008 